Florida International University

FIU Digital Commons

FIU Electronic Theses and Dissertations

University Graduate School

3-29-2018

\title{
Multimodal Imaging for Enhanced Diagnosis and for Assessing Progression of Alzheimer's Disease
}

Chunfei Li

Florida International University, cli029@fiu.edu

Follow this and additional works at: https://digitalcommons.fiu.edu/etd

Part of the Biomedical Commons

\section{Recommended Citation}

$\mathrm{Li}$, Chunfei, "Multimodal Imaging for Enhanced Diagnosis and for Assessing Progression of Alzheimer's Disease" (2018). FIU Electronic Theses and Dissertations. 3703.

https://digitalcommons.fiu.edu/etd/3703

This work is brought to you for free and open access by the University Graduate School at FIU Digital Commons. It has been accepted for inclusion in FIU Electronic Theses and Dissertations by an authorized administrator of FIU Digital Commons. For more information, please contact dcc@fiu.edu. 


\title{
FLORIDA INTERNATIONAL UNIVERSITY
}

Miami, Florida

\section{MULTIMODAL IMAGING FOR ENHANCED DIAGNOSIS AND FOR ASSESSING PROGRESSION OF ALZHEIMER'S DISEASE}

A dissertation submitted in partial fulfillment of

\author{
the requirements for the degree of \\ DOCTOR OF PHILOSOPHY \\ in \\ ELECTRICAL ENGINEERING \\ by
}

Chunfei Li

2018 
To: Dean John L. Volakis

College of Engineering and Computing

This dissertation, written by Chunfei Li, and entitled Multimodal Imaging for Enhanced Diagnosis and for Assessing Progression of Alzheimer's Disease, having been approved in respect to style and intellectual content, is referred to you for judgment.

We have read this dissertation and recommend that it be approved.

Jean Andrian

Armando Barreto

Mercedes Cabrerizo

Naphtali David Rishe

Malek Adjouadi, Major Professor

Date of Defense: March 29, 2018

The dissertation of Chunfei Li is approved.

Dean John L. Volakis

College of Engineering and Computing

Andrés G. Gil

Vice President for Research and Economic Development and Dean of the University Graduate School

Florida International University, 2018 
C) Copyright 2018 by Chunfei Li

All rights reserved. 


\section{ACKNOWLEDGMENTS}

I would like to express my most sincere gratitude to my major advisor, Dr. Malek Adjouadi for his encouragement, patience and guidance in my research and for his kindness and consideration throughout the past four years that I have worked with him with the Center for Advanced Technology and Education-CATE. With his continuous support and invaluable academic advice, I was able to get over all the difficulties and finish my $\mathrm{PhD}$ studies and realize my goal.

Special thanks go to all the kind persons that helped me throughout the past four years. I am grateful to Dr. Ranjan Duara from Mount Sinai Medical Center and Dr. David Loewenstein from the University of Miami for their valuable suggestions in all clinical and neuropsychological aspects of the Alzheimer's disease. Many thanks go to my committee members Dr. Mercedes Cabrerizo, Dr. Armando Barreto, Dr. Jean Andrian, and Dr. Naphtali David Rishe for their professional support and assistance.

I acknowledge the critical support provided by the National Science Foundation under grants: CNS-1532061, CNS-1551221, CNS-1642193, CNS-0959985, HRD-0833093, IIP 1338922, and CNS-1429345. The generous support of the Ware Foundation and the FIU UGS Dissertation Year Fellowship are also appreciated.

I appreciate the support provided by the Florida-based Alzheimer's Disease Research Center (ADRC) (1P50AG047266-01A1), R01 AG047649-01A1 (David Loewenstein, PI), the Florida Department of Health, Ed and Ethel Moore Alzheimer's Disease Research Program, and the Wien Center for Alzheimer's Disease \& Memory Disorders, Mount Sinai Medical Center, Miami, FL. 


\title{
ABSTRACT OF THE DISSERTATION \\ MULTIMODAL IMAGING FOR ENHANCED DIAGNOSIS AND FOR ASSESSING \\ PROGRESSION OF ALZHEIMER'S DISEASE
}

\author{
by \\ Chunfei Li \\ Florida International University, 2018 \\ Miami, Florida

\section{Professor Malek Adjouadi, Major Professor}

A neuroimaging feature extraction model is designed to extract region-based image features whose values are predicted by base learners trained on raw neuroimaging morphological variables. The main objectives are to identify Alzheimer's disease (AD) in its earliest manifestations, and be able to predict and gauge progression of the disease through the stages of mild cognitive impairment (EMCI), late MCI (LMCI) and AD. The model was evaluated on the ADNI database and showed $75.3 \%$ accuracy for the challenging EMCI diagnosis based on the 10-fold cross validation. Our approach also performed well for the other binary classifications: EMCI vs. LMCI (72.3\%), EMCI vs. $\mathrm{AD}(95.0 \%), \mathrm{LMCI}$ vs. AD (84.3\%), CN vs. LMCI (77.5\%), and CN vs. AD (96.5\%). By applying the model to the Genome-wide Association Study, along with the sparse Partial Least Squares regression method, we successfully detected risk genes such as the APOE, TOMM40, RVRL2 and APOC1 along with the new finding of rs917100.

Moreover, the research aimed to investigate the relationship of different biomarkers; especially the imaging biomarkers to better understand the precise biologic changes that 
characterize Alzheimer's disease. The unique and independent contribution of APOE4 allele status (E4+\E4-), amyloid (A $\beta$ ) load status (Amy+\Amy-) and combined APOE4 and $\mathrm{A} \beta$ status on regional cortical thickness $(\mathrm{CTh})$ and cognition were evaluated via a series of two-way ANCOVAs with post-hoc Tukey HSD tests. Results showed that decreased CTh is independently associated with Amy+ status in many brain regions, but with E4+ status in very restricted number of brain regions. Among $\mathrm{CN}$ and EMCI participants, E4+ status is associated with increased $\mathrm{CTh}$, in medial and inferior temporal regions.

Diverging association patterns of global and regional A $\beta$ load with cortical volume were found in the entorhinal, temporal pole and parahippocampal regions, which were positively associated with regional $A \beta$ load, but with a negative correlation for global $A \beta$ load in MCI stages.

In addition, strong positive correlations were shown between baseline regional CTh and the difference of $\mathrm{CTh}$ in each region between the $\mathrm{CN}$ and $\mathrm{AD}$, even after adjusting for the regional $\mathrm{A} \beta$ and APOE genotype $(\mathrm{E} 4+: \mathrm{r}=0.521$ and $\mathrm{E} 4-\mathrm{r}=0.694)$. 
TABLE OF CONTENTS

CHAPTER

PAGE

INTRODUCTION 1

CHAPTER I

INVESTIGATING THE UTILITY OF FDG-PET AND AV45-PET IN CLASSIFICATION OF ALZHEIMER'S DISEASE AND ITS PRODROMAL STAGES 7

CHAPTER II

ENHANCED REGION-BASED NEUTOIMAGING FEATURES FOR THE EARLY

DETECTION OF ALZHEIMER'S DISEASE 13

CHAPTER III

REGIONAL IMAGE FEATURES MODEL FOR THE GENOME-WIDE ASSOCIATION STUDY OF ALZHEIMER'S DISEASE 26

CHAPTER IV

THE RELATIONSHIP OF BRAIN AMYLOID LOAD AND APOE STATUS TO REGIONAL CORTICAL THINING AND COGNITION....

CHAPTER V

DIVERGING ASSOCIATION PATTERNS OF REGIONAL CORTICAL ATROPHY WITH GLOBAL AND WITH REGIONAL AMYLOID DEPOSITION.

CHAPTER VI

PATTERN ANALYSIS OF THE INTERACTION OF REGIONAL AMYLOID, CORTICAL THICKNESS, AND APOE GENOTYPE IN THE PROGRESSION OF ALZHEIMER'S DISEASE

CHAPTER VII

REGIONALSHIP BETWEEN REGIONAL CORTICAL THICKNESS, AMYLOID LOAD AND SELECTIVE VULNERABILITY TO ATROPHY IN ALZHEIMER'S DISEASE 


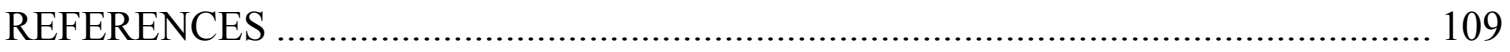

VITA

viii 


\section{LIST OF TABLES}

TABLE

PAGE

Table 1. Participant demographic information ............................................................ 8

Table 2. P-values of multiple linear regression models .................................................. 10

Table 3. Accuracy of SVM using 10-fold cross validation (\%) ...................................... 11

Table 4. Participants' demographic information ............................................................ 14

Table 5. Comparison of the classification accuracy (ACC), sensitivity (SEN), and specificity (SPE) (\%) values of baseline with proposed feature extraction based model for 6 different pairs of binary classification

Table 6. Comparison of the classification accuracy (ACC), sensitivity (SEN), and specificity (SPE) values of the proposed model with others for 6 different pairs of binary classification

Table 7. Participants demographic information for the imaging genetic study ................ 28

Table 8. The 43 Statistical summaries for each region................................................... 29

Table 9. Fifteen SNPs Selected by sPLS model …………………………………...... 35

Table 10. Participant clinical information .................................................................... 39

Table 11. Effect of APOE4 status on global amyloid load (SUVR) in different

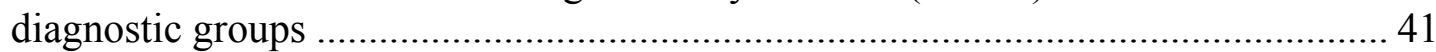

Table 12. Effect of amyloid status on regional CTh, independent of APOE4 status (left

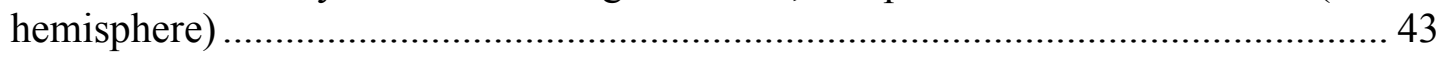

Table 13. Effect of APOE4 status on regional CTh, independent of A $\beta$ load (left

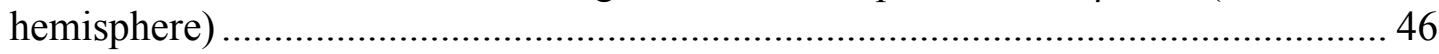

Table 14. Effect of APOE4 status on regional $\mathrm{CTh}$ in $\mathrm{CN}$ and EMCI, independent of A $\beta$ load

Table 15. Combined effect of APOE4 status and A $\beta$ load status on cognitive scores

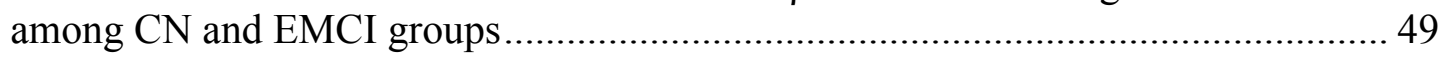

Table 16. Associations of cortical atrophy and global $A \beta$ load........................................ 64

Table 17. Associations of cortical atrophy and global $A \beta$ load (corrected $p$ value)......... 65

Table 18. Associations of cortical atrophy and regional $A \beta$ load..................................... 67 
Table 19. Associations of cortical atrophy and global A $\beta$ load (corrected $p$ value)........ 72

Table 20. Age, gender-matched participants' demographic information ........................ 91

Table 21. CTh in the $\mathrm{CN}$ and AD Groups, \% mean differences between $\mathrm{CN}$ and $\mathrm{AD}$ groups $\left(\% \mathrm{CTh}^{\text {Diff }}\right)$ and regional $\mathrm{A} \beta$ load $\left(\mathrm{rSUVR}^{\mathrm{CN}}\right)$, for $\mathrm{E} 4+$ subjects in the left

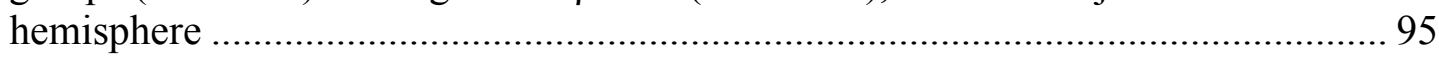

Table 22. CTh in the $\mathrm{CN}$ and AD Groups, \% mean differences between $\mathrm{CN}$ and $\mathrm{AD}$ groups $\left(\% \mathrm{CTh}^{\text {Diff }}\right)$ and regional $\mathrm{A} \beta$ load $\left(\mathrm{rSUVR}^{\mathrm{CN}}\right)$, for E4- subjects in the left

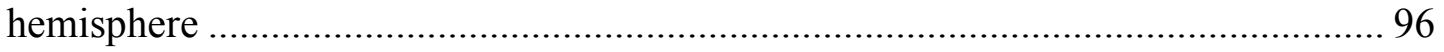

Table 23. Associations of $\mathrm{rCTh}^{\mathrm{CN}}$ with $\% \mathrm{CTh}^{\text {Diff }}$ before and after separating out the

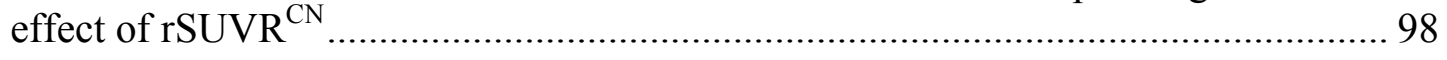

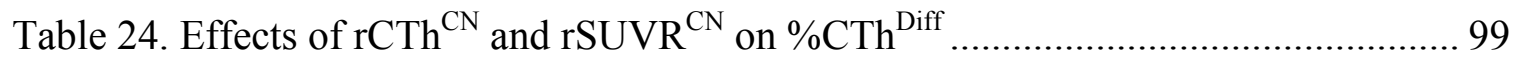




\section{LIST OF FIGURES}

FIGURE

PAGE

Fig. 1. The five individual MetaROI used in 18F-FDG. ........................................... 9

Fig. 2. Region-based neuroimang feature extraction model illustration for the left entorhinal ROI in the $\mathrm{CN}$ vs. EMCI classification...... 19

Fig. 3. ROC curves of different imaging biomarkers and the proposed imaging feature of the left entorhinal ROI for the $\mathrm{CN}$ vs. EMCI classification. 23

Fig. 4. The flowchart illustrating the genome-wide association study using proposed ROI-based feature extraction model

Fig. 5. Principal Component Analysis. 34

Fig. 6. The accuracy of 50 final SVM(Radial) Models (10-fold cross validation). 34

Fig. 7. Heat map of the selected 15 SNPs by sPLS using extracted image features as responses. 36

Fig. 8. Differences in mean amyloid load $\left({ }^{18} \mathrm{~F}-\mathrm{AV} 45\right) \mathrm{SUVR}$ for each diagnostic group between E4- and E4+ participants.

Fig. 9. Barplot of CTh among Amy- and Amy+ participants in 4 diagnostic groups for 12 brain regions.

Fig. 10. Barplot of CTh among E4- and E4+ participants in 4 diagnostic groups for 12 brain regions.

Fig. 11. Bar graph of scores on following cognitive tests: MMSE, RAVLT

(immediate), RAVLT ( $\%$ forgetting) and ADAS13.

Fig. 12. Association patterns of cortical atrophy with global $A \beta$ load (A), and regional $A \beta$ load (B), displayed as heatmap with partial correlation coefficients displayed at $\mathrm{p}$ (uncorrected) $<0.05$.

Fig. 13. Linear regression models and performance of volume, regional A $\beta$ load and global A $\beta$ load in right precuneus..

Fig. 14. Association patterns of cortical atrophy with global amyloid load (A), and regional $A \beta$ load $(B)$, displayed as heatmap with partial correlation coefficients displayed at $\mathrm{p}$ (corrected $)<0.05$.

Fig. 15. Linear regression models and performance of volume, regional A $\beta$ load and global A $\beta$ load in right parahippocampal 
Fig. 16. Linear regression models and performance of volume, regional A $\beta$ load and global $A \beta$ load in right entorhinal..

Fig. 17. Associations between regional and global A $\beta$ load. The fitted curves are from LOESS regression models for all subjects, with smoothing degree equals to $0.4 \ldots . . .77$

Fig. 18. Complete linkage hierarchical clustering illustration....................................... 83

Fig. 19. Heatmap representation of clustering analysis.. ....................................... 86

Fig. 20. Scatterplot, linear regression as well as boxplot of (a) right precentral, (b) left

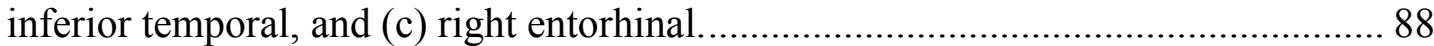

Fig. 21. Plot of $\mathrm{rCTh}^{\mathrm{CN}}$ and $\% \mathrm{CTh}^{\text {Diff }}$ with the estimated linear regression model for

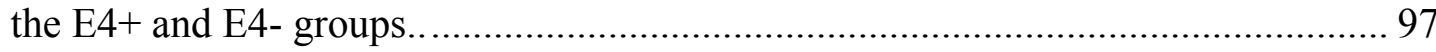

Fig. 22. Plot of $\mathrm{rSUVR}^{\mathrm{CN}}$ and $\% \mathrm{CTh}^{\mathrm{Diff}}$ with the estimated linear regression model for the E4+ and E4- groups.. 103

Fig. 23. Plot of $\mathrm{rCTh}^{\mathrm{CN}}$ and $\% \mathrm{CTh}^{\text {Diff }}$ with the estimated linear regression model for the global amyloid positive (gSUVR+) and negative (gSUVR-) groups................ 104 


\section{INTRODUCTION}

Alzheimer's disease is a neurodegenerative disorder characterized by the progressive loss of neural cells, affecting parts of the brain that control thought, memory, and sometimes language. In 2011, the National Institute on Aging (NIA) and the Alzheimer's Association proposed revised criteria and guidelines for diagnosing Alzheimer's disease, and three stages of Alzheimer's disease were identified: preclinical Alzheimer's disease, mild cognitive impairment (MCI) due to Alzheimer's disease, and dementia due to Alzheimer's disease (AD) [1-4]. In the preclinical Alzheimer's disease stage, individuals have not yet developed noticeable symptoms such as memory loss, but do have some noticeable changes in the brain. In the MCI stage, which is subdivided into early MCI (EMCI) and late MCI (LMCI) by the Alzheimer's disease Neuroimaging Initiative (ADNI), people have mild symptoms in thinking abilities but can still perform everyday tasks. Studies show that about $10-12 \%$ of subjects with MCI progress to AD per year [5]. However, MCI does not always lead to dementia. In some individuals, MCI reverts to normal cognition or remains stable. The AD stage is characterized by quite noticeable memory, thinking and behavioral symptoms that impair a person's ability to function in daily life.

As the population of the United States ages, Alzheimer's is becoming a more common cause of death. According to data from the National Center for Health Statistics of the Centers for Disease Control and Prevention (CDC), between 2000 and 2013, deaths attributed to Alzheimer's disease increased 71 percent, while those attributed to other major causes (breast cancer, prostate cancer, heart disease, stroke, and HIV, etc.) have 
decreased significantly [6]. In 2013, 84,767 people died from AD, and an estimated 700,000 people in the United States age 65 and older will die with AD based on the Chicago Health and Aging Project (CHAP) [7]. Due to the aging of the population and with more people living longer, the number of individuals with Alzheimer's disease is projected to double by 2050 . Thus, early and reliable detection is believed to be the key to preventing, slowing and potentially stopping Alzheimer's disease.

Alzheimer's disease (AD) is associated with the excessive aggregation of amyloid beta protein $(A \beta)$ outside neurons and tau protein inside nerve cells. Neuropathological diagnostic criteria for $\mathrm{AD}[8]$ require the deposition of $\mathrm{A} \beta$, which accumulates initially and most prominently in neocortical regions, such as the precuneus, posterior and anterior cingulate gyrus, and the orbitofrontal cortex, and tau-associated neurofibrillary tangles $[9$, 10]. Neurofibrillary tangle pathology in $\mathrm{AD}$, in contrast to $\mathrm{A} \beta$-associated pathology, follows a stereotypical topographic pattern initially involving the most selectively vulnerable regions, such as the entorhinal cortex (ERC) and parahippocampal gyrus, and then progressing to limbic and finally association cortices [11].

According to the amyloid hypothesis [8], deposition of amyloid beta protein (A $\beta)$ in the neocortex, is the initiating event in the pathophysiology of Alzheimer's disease (AD) and occurs 15 to 20 years before the first symptoms of the disease. This leads to downstream events including neurodegeneration and ultimately cognitive and functional impairment. Recent neuropathological diagnostic criteria for $\mathrm{AD}$ are based upon this hypothesis, incorporating the Thal phase schema of a stereotypic pattern of $A \beta$ accumulation, 
anteceding Braak staging of neurofibrillary tangle pathology in the brain, with a continuous relationship existing between brain $A \beta$ load and neurodegenerative changes [9].

Another insight into the $\mathrm{AD}$ can be gained by exploring its genetic foundation. The single-nucleotide polymorphism (SNP), which is the most common and stable type of the DNA sequence variations, has commonly been used to analyze and identify complex neurological diseases such as AD. So far, the apolipoprotein E (APOE) gene is one of the prevalent risk factor that has been shown to have a strong connection with AD. More precisely, among its three variants alleles, APOE e2, APOE e3, and APOE e4 (APOE4), APOE4 is found to be the one most associated with increased risk for AD [12-17]. Some other genes such as TOMM40 [18, 19], CLU [20] and PVRL2 [21] are also considered to be significant risk factors.

There is also considerable evidence that APOE E4 (APOE4) carrier (E4+) status is associated with greater $A \beta$ load in normal individuals as well as in all stages of $A D$, possibly as a result of the effect of APOE4 genotype on impaired clearance of A $\beta$ protein $[22,23]$. Aging and E4+ status are among the most strongly associated factors with increased risk for $\mathrm{AD}$ [22-24]. Recent clinical criteria for the diagnosis of $\mathrm{AD}$ dementia and Prodromal AD (NIA-AA and IWG criteria) rely on combinations of "positive biomarkers" in the presence of functional and/or cognitive impairment with high, intermediate or low levels of likelihood $[2,25]$ based on the presence of $A \beta$ and 
neurodegenerative biomarkers. The presence of $A \beta$ biomarkers in the absence of cognitive and functional impairment fulfills criteria for a diagnosis of preclinical AD.

Neuroimaging is an important research platform for understanding the complicated pathogenesis of Alzheimer's disease (AD). Deposition of $A \beta$ in vivo is detectable with positron emission tomography (AV45 PET) scans, using an A $\beta$ binding ligand, or by measuring $A \beta$ levels in the cerebrospinal fluid (CSF), whereas downstream events such as neurodegeneration are detectable using volumetric measures of regional atrophy (especially hippocampal atrophy) and reduced cortical thickness (CTh) on structural magnetic resonance imaging (MRI) scans, as well as deficits in regional cerebral glucose metabolism on PET scans (FDG PET). Compared with the MRI, the cost of PET scans is extremely high; hence not every subject underwent all types of the PET, such as the AV45 and FDG PET.

In recent years, machine learning and deep learning techniques have been widely performed in the diagnosis of Alzheimer's Disease (AD), and its prodromal stage, mild cognitive impairment (MCI), and reached very high diagnostic accuracy. It is believed that, combinations of different biomarkers could improve the classification performance. However, this will lead one of the main challenges in the area of imaging-based diagnosis, which is the extremely high dimensionality of image data (hundreds of morphological variables or millions of voxels), albeit with a relatively small number of subjects in $\mathrm{AD}$ research (few hundreds). To reduce the feature dimensions, some researchers proposed features selection pipeline such as pre-selecting brains regions 
sensitive to $\mathrm{AD}$ (e.g. entorhinal cortex, parahippocampal gyrus, and hippocampus), or ranking features with regrads to their discrimicate significant difference between $\mathrm{CN}$ and $\mathrm{AD} / \mathrm{MCI}$ [26-31]. Alternatively, others performed feature extraction frameworks to map the original high dime to the lower new space. Such methods include principle component analysis (PCA), linear discriminant analysis, and Independent Component Analysis (ICV) [32-36]. Recently, deep learning techniques are widely used in the AD classification at the voxel level with the hypothesis that predefined regions could potentially downgrade the power of the biomarker to detect differences or changes over time[35, 37-45]. However, only few studies have mentioned the performance on the diagnosis of EMCI so far [31, 35, 38, 39, 43, 46].

On the other hand, although it is well accepted that A $\beta$ load, APOE4 status and neurodegeneration are strongly interrelated [47], the presence and strength of the relationships between these factors and their independent effects on cortical thinning and cognition are not well understood at different stages of disease. As emerging treatments are developed, it is increasingly important to understand these independent relationships prior to developing appropriate disease modifying treatments for AD.

In this dissertation, we aim to early identify Alzheimer's disease, and predict the progression in $\mathrm{MCI}$ and $\mathrm{AD}$ by incorporating imaging and any other biomarkers, results of cognitive tests and patient's medical history in the most effective way with the hypothesis that combinations of different biomarkers could improve the classification performance. Also, the research aims to investigate the relationship of different 
biomarkers, especially the imaging biomarkers to better understand the precise biologic changes that cause Alzheimer's disease, which helps to developing appropriate disease modifying treatments for $\mathrm{AD}$.

Specifically, the performance of the AV45 PET and the FDG PET on the diagnosis of the different stages was first investigated in Chapter 1 . And the one with better performance in the early stage of $\mathrm{AD}$ was selected and used in the following studies. In Chapter 2, we proposed a region-based neuroimaging extraction model to assist the diagnosis of the $\mathrm{AD}$ and its prodromal stage. The proposed feature extraction model was also used in the Genome-wide Association Study of Alzheimer's Disease as explained in Chapter 3. Regarding the relationship of different imaging biomarkers, the association of different types of imaging biomarkers, APOE4 gene and the cognitive performance were thoroughly studied Chapter 4; the association patterns of regional cortical atrophy with global and with regional amyloid deposition in the progression of $\mathrm{AD}$ were explored in Chapter 5 and Chapter 6. Finally, the relationships between the baseline regional cortical thickness, baseline regional amyloid load and selective vulnerability to atrophy is examined in Chapter 7. 


\section{CHAPTER I}

\section{INVESTIGATING THE UTILITY OF FDG-PET AND AV45-PET IN \\ CLASSIFICATION OF ALZHEIMER'S DISEASE AND ITS PRODROMAL \\ STAGES}

\subsection{Goal}

This study is aimed to explore the independent and interactive effects of the two commonly used 18F amyloid radiotracers (AV45 and FDG) on discriminating Alzheimer Disease (AD), early and late mild cognitive impairment (EMCI and LMCI) from cognitive normal $(\mathrm{CN})$. It's also aimed to examine the diagnostic power of the two PET radiotracers based on binary classification and multi-classification methods.

\subsection{Materials and Methods}

\subsubsection{Data}

Data used in the preparation of this article were obtained from the ADNI database (adni.loni.usc.edu). The ADNI was launched in 2003 as a public-private partnership, led by Principal Investigator Michael W. Weiner, MD. The primary goal of ADNI has been to test whether serial magnetic resonance imaging (MRI), positron emission tomography (PET), other biological markers, and clinical and neuropsychological assessment can be

combined to measure the progression of mild cognitive impairment (MCI) and early Alzheimer's disease (AD). 
For this first study, 508 subjects (CN (135), EMCI (208), LMCI (208), or AD (102)) underwent both 18F-AV45 Amyloid PET scan and 18F-FDG PET scan were included in this study. The Baseline demographic information was shown as shown in Table 1.

Table 1. Participant demographic information

\begin{tabular}{llllll}
\hline & CN & EMCI & LMCI & AD & p-value $^{\text {b }}$ \\
\hline Number of Subjects (n) & 135 & 208 & 102 & 63 & N/A \\
Gender (Male/Female) & $65 / 70$ & $112 / 96$ & $54 / 48$ & $36 / 27$ & 0.632 \\
Age (Years) & $73.7(6.0)^{\mathrm{a}}$ & $70.0(6.9)$ & $71.2(7.8)$ & $75.1(8.0)$ & $<0.0001$ \\
Education (Years) & $16.4(2.5)$ & $16.0(2.7)$ & $16.5(2.7)$ & $15.9(2.5)$ & 0.291 \\
\hline
\end{tabular}

\footnotetext{
${ }^{a}$ Unless otherwise noted, data presented as mean (standard deviation).

${ }^{b}$ The $p$ values of ANOVA testing between the four groups were shown, and age is found to be a significant factor.
}

\subsubsection{PET Analysis}

A detailed description of PET protocols and acquisition procedures can be found in the following link: http://adni.loni.usc.edu/methods/pet-analysis/pre-processing. Briefly, all PET images were acquired 30 to 60 minutes post-injection, co-registered, averaged, reoriented into a standard $160 \times 160 \times 96$ voxel image grid, and smoothed to a uniform isotropic resolution of $8 \mathrm{~mm}$ full width of maximum. 18F-FDG: Normalized intensity of the five pre-defined regions of interest (MetaROIs) - Post Cingulate Gyrus, Left Angular Gyrus, Right Angular Gyrus, Left Temporal Gyrus and Right Temporal Gyrus - as well as a composite region made up of all five regions were used as the 6 classification features. 18F-AV45: Mean standardized uptake value ratios (SUVRs) for four cortical 
regions (frontal, anterior/posterior cingulate, lateral parietal, and lateral temporal) and three reference regions (cerebellar grey matter, whole cerebellum, and brainstem/pons) were used as the additional 7 classification features (Fig. 1).

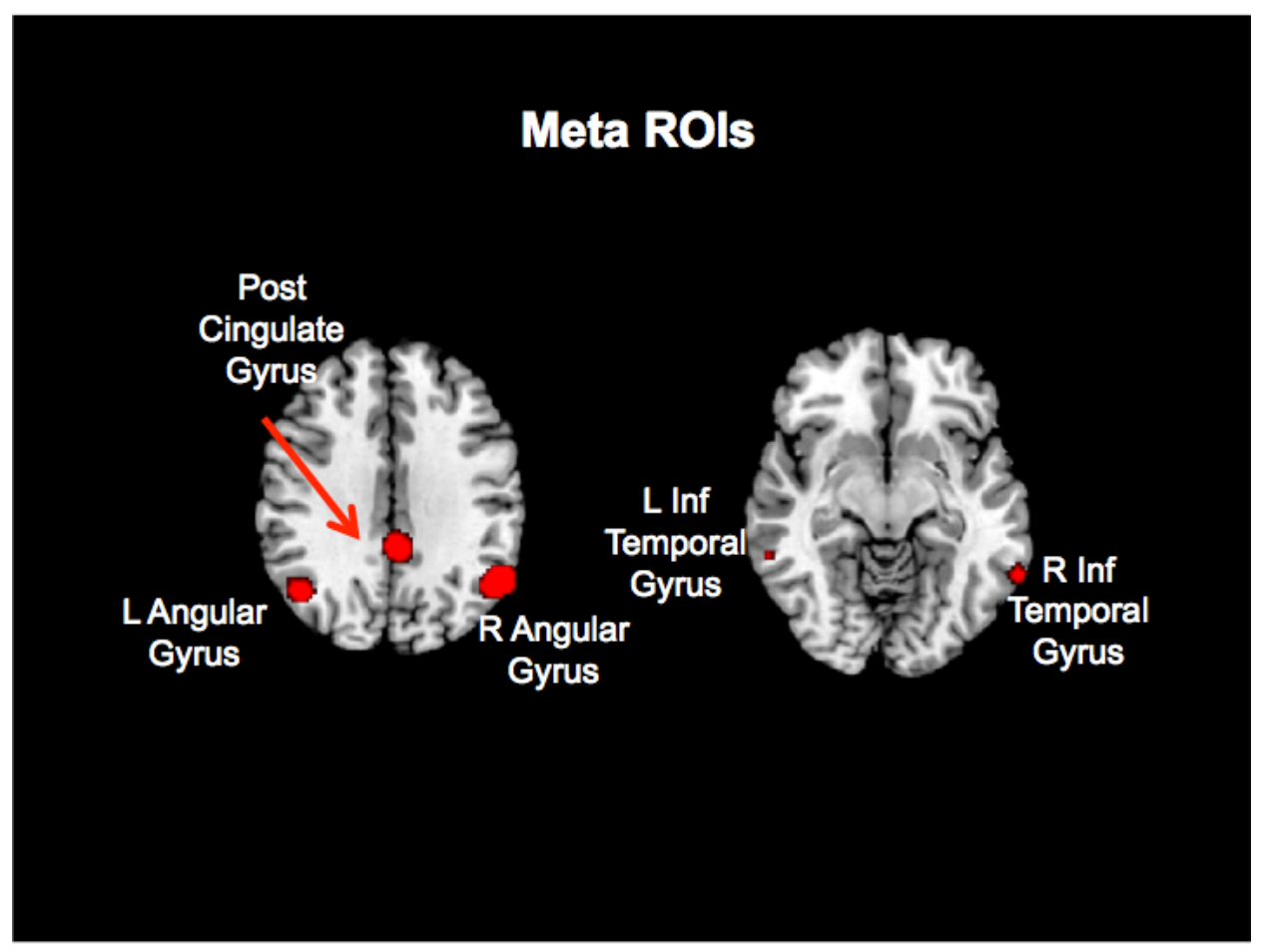

Fig. 1. The five individual MetaROI used in 18F-FDG.

\subsubsection{Investigation of FDG, AV45 and Their Interactive Effect}

Two multiple linear regression models - additive and non-additive were performed to determine the contribution of each radiotracer and identify if there is a significant interaction between them. The difference of the two models is that the additive model assumes independence of two biomarkers, while non-additive model considers the interaction of the two as well. The features used in this step were the average FDG-PET for 5 MetaROIs and the average AV45 SUVR for the 4 aforementioned ROIs. 


\subsubsection{Classification Experiment}

Support vector machine (SVM) classifier with a kernel of Gaussian radial basis function was applied to FDG and AV45 data separately and combined. The experiment is designed for both two-classification and multi-classification with 10-fold crossvalidation.

\subsection{Results}

The results of investigating the interactive effect of the two biomarkers are shown in Table 2, which shows that in the additive model, both biomarkers are significant. However, in the non-additive model, FDG, when taken out of the interactive effect with AV45, is no longer significant. As expected, the interaction of the two biomarkers in the non-additive model are significant.

Table 2. P-values of multiple linear regression models

\begin{tabular}{lll}
\hline PET radiotracers & Non- additive model & Additive model \\
\cline { 2 - 3 } & $\operatorname{lm}\left(D X \sim A V 45_{-} b l+F D G_{-} b l+A V 45_{-} b l: F D G_{-} b l\right)$ & $\operatorname{lm}\left(D X \sim A V 45_{-} b l+F D G_{-} b l\right)$ \\
\hline AV45 & $<0.001$ & $<0.001$ \\
FDG & 0.105 & $<0.001$ \\
Interaction & $<0.001$ & N/A \\
\hline
\end{tabular}

As can be seen from the results given in Table 3, FDG outperforms AV45 in most of the compared two-classification cases except for $\mathrm{CN}$ vs. LMCI and $\mathrm{CN}$ vs. EMCI. It may be deduced that FDG is more capable of capturing patterns of $\mathrm{AD}$, while biomarker AV45 detects patterns of prodromal stage of AD more efficiently. It also shows that combining both biomarkers doesn't have a significant increase in accuracy when compared with the 
results using either of them. In particular, while discriminating AD from CN, FDG biomarker alone achieved $90.11 \%$ accuracy, and this accuracy increased to $92.67 \%$ when using both biomarkers. Multi-classification proved more challenging, and the results suggest that combining the two biomarkers still achieves a higher performance.

It may be inferred from the results in Tables 2 and 3 that even as FDG displays much higher discriminative efficiency between $\mathrm{AD}$ and $\mathrm{CN}$, however, when taking out the interaction effect of the two biomarker, FDG may not be able to discriminate EMCI and/or LMCI from $\mathrm{CN}$ anymore, and is somewhat inconsequential in the non-additive model as shown in Table 2.

Table 3. Accuracy of SVM using 10 -fold cross validation (\%)

\begin{tabular}{lccc}
\hline & AV45 & FDG & AV45 and FDG \\
\hline Two-group Classification & & & \\
CN vs. AD & 83.59 & 90.11 & 92.67 \\
CN vs. LMCI & 74.42 & 71.47 & 75.44 \\
CN vs. EMCI & 65.09 & 61.17 & 62.99 \\
EMCI vs. AD & 81.16 & 88.23 & 87.08 \\
EMCI vs. LMCI & 68.06 & 70.32 & 70.65 \\
LMCI vs. AD & 62.39 & 73.93 & 74.45 \\
\hline Multi-group Classification & & & \\
CN vs. EMCI vs. LMCI vs. AD & 45.64 & 48.06 & 50.4 \\
\hline
\end{tabular}

\subsection{Conclusion}

This study, by establishing two multiple linear regression models (additive and nonadditive), confirmed previous findings that the two biomarkers (AV45 and FDG) are highly correlated with respect to the diagnosis. The results also indicate that AV45 
outperforms FDG in case of EMCI vs. $\mathrm{CN}$ and $\mathrm{LMCI}$ vs. CN, while FDG is more effective than AV45 in separating AD from $\mathrm{CN}$ as well as from its prodromal stages EMCI and LMCI. When using both the two-group and multi-group classifications, combining FDG and AV45 slightly improves the classification accuracy. 


\section{CHAPTER II}

\section{ENHANCED REGION-BASED NEUTOIMAGING FEATURES FOR THE EARLY DETECTION OF ALZHEIMER'S DISEASE}

\subsection{Goal}

In this study, we present a region-based neuroimaging biomarker extraction model based on shape and functional features from MRI and PET imaging to fuse the information associated with the disease, i.e. the probability of being EMCI. The extracted biomarkers along with patients' demographic information such as age, gender and APOE status are used as the predictors in the EMCI classification model. The proposed framework was also evaluated on the other binary classification tasks: $\mathrm{CN}$ vs. LMCI, CN vs. AD, EMCI vs. LMCI, EMCI vs. AD, and LMCI vs. AD.

\subsection{Materials and Method}

\subsubsection{Data}

For this second study, 893 subjects who underwent MRI and their first AV45 PET scans within 6 months and with available APOE gene information were involved in this study. They were categorized into groups of CN (247), EMCI (295), LMCI (193) and AD (158) according to ADNI diagnosis criteria, which assessed individual's Mini-Mental State Examination (MMSE) score, Clinical Dementia Rating (CDR) score and education adjusted score on delayed paragraph recall from Wechsler Memory Scale Logical Memory II. Table 4 provides the demographics of the study subjects. 
Table 4. Participants' demographic information

\begin{tabular}{lllllll}
\hline & CN & EMCI & LMCI & AD & F-value $^{\text {a }}$ & P-Value $^{\text {P-Val }}$ \\
\hline $\mathrm{N}$ & 247 & 295 & 193 & 158 & & \\
Female/Male & $125 / 122$ & $131 / 164$ & $83 / 110$ & $67 / 91$ & 3.84 & 0.280 \\
APOE4 0/1/2 & $180 / 61 / 6$ & $168 / 109 / 18$ & $92 / 75 / 26$ & $52 / 74 / 32$ & 85.2 & $<0.001$ \\
Age (year) & $75.3(6.5)^{\text {b }}$ & $71.3(7.4)$ & $73.6(8.1)$ & $74.7(7.8)$ & 14.72 & $<0.001$ \\
Education (year) & $16.4(2.6)$ & $16(2.7)$ & $16.3(2.7)$ & $15.8(2.7)$ & 2.4 & 0.067 \\
MMSE & $29(1.2)$ & $28.3(1.6)$ & $27.6(1.9)$ & $22.8(2.7)$ & 433.1 & $<0.001$ \\
\hline
\end{tabular}

${ }^{a}$ F-values are for ANOVA test (continuous attribute: Age, Education, and MMSE) or Chi-square test ( categorical attribute: gender and APOE genotype), significance level is 0.05 by default

${ }^{\mathrm{b}}$ Values are represented as mean(standard deviation) for all continuous attributes

\subsubsection{Neuroimaging Acquisition}

In terms of MRI scans, the MPRAGE files as used in our study had undergone the following correction steps: (i) Gradwarp: corrected image geometry distortion due to gradient non-linearity (for MRI obtained by GE and Siemens systems only); (ii) B1 correction: employed the B1 calibration scans to correct the image intensity nonuniformity (for GE and Siemens systems with receive-only head RF coils only); and (iii) N3: applied the N3, a histogram peak sharpening algorithm, to 3T MRI (ADNI 1/GO/2) to reduce intensity non-uniformity due to the wave or the dielectric effect and 1.5T MRI (ADNI 1) to reduce residual intensity non-uniformity (for Phillips Systems and GE and Siemens systems). The resulting pre-processed MRIs were labeled with "N3" in ADNI.

Each subject's first AV45 PET scan was selected along with the structural MRI that was closest in time to PET acquisition. Details of AV45 PET and MRI imaging acquisition and pre-processing steps can be found in the aforementioned ADNI website. In brief, the ADNI had already performed the attenuation correction for all PET scans. And the PET 
image was then preprocessed following these specific steps: (i) co-registered the extracted 5 five-minute frames to the first extracted frame from raw PET to lessen the effects of patient motion; (ii) averaged the co-registered dynamic 6 frames to create a single 30 min PET image; (iii) reoriented the 30 min PET to a standard 160x160x96 voxel image grid with $1.5 \mathrm{~mm}$ cube voxels and normalized the intensity of the PET scan; and (iv) smoothed the above image with a scanner-specific filter, and obtained our preprocessed PET scan of a uniform isotropic resolution of $8 \mathrm{~mm}$ Full Width at Half maximum (FWHM), which was identified with "AV45 Coreg, Avg, Std Img and Vox Siz, Uniform Resolution” in ADNI.

\subsubsection{MRI Processing}

FreeSurfer pipeline (version 5.3.0) [48] was applied to the MRI scans under centos4_x86_64 Linux system to produce cortical and subcortical volumetric variables. The original MRI scan was first mapped to the standard MNI 305 space, yielding the image referred to as T1.mgz, which was used as the reference image in the following registration procedure. Based on the $\mathrm{T} 1$ image, the corresponding image file termed as aparc+aseg.mgz provides the FreeSurfer parcellated and segmented cortical and subcortical regions. CTh, surface area and volume were then calculated as morphological variables on each of the 34 cortical regions for both hemispheres as well as the volume on each of the 45 subcortical regions of the whole brain.

Multiple morphological variables were then generated for the labeled regions. Specially, there are 9 measurements for each cortical regions: surface area, gray matter volume, 
average thickness, thickness standard deviation, integrated rectified mean curvature, integrated rectified Gaussian curvature, folding index, intrinsic curvature index, and white matter volume, and 3 measurements for the subcortical regions: volume, mean intensity and intensity standard deviation. The estimated total intracranial volume (ICV) is also provided.

\subsubsection{PET processing}

In order to quantify the A $\beta$ load from the PET scans, FMRIB Software Library (FSL) [49] was then used to co-register the PET image to the aforementioned T1 image. Considering the relatively low resolution of the PET image and to utilize as much information from PET as possible, the AV45 PET scan, with the skull, were co-registered linearly (i.e., trilinear interpolation) with 12 degrees of freedom (DOF) onto the T1 image. Such a registration process guaranteed that the AV45 PET image had the same accurate segmentation and parcellation as in the MRI. Thus, the mean $A \beta$ load of each of the FreeSurfer defined regions can be calculated, which was used later to calculate the global A $\beta$ load value.

\subsubsection{Global A $\beta$ Load Calculation}

The registered AV45 PET with the aparc+aseg image was first inspected to ensure appropriate calculations of the mean A $\beta$ uptake value (SUV) of all the FreeSurfer-defined regions (ROIs) as expressed in (2.1). 


$$
S U V_{R_{O I I_{k}}}=\frac{\sum_{i=1}^{N_{R O I_{k}}} V a l_{i}}{N_{R O I_{i}}}
$$

where $S U V_{R O I_{k}}$ represents the mean $\mathrm{A} \beta$ uptake value of the region $R O I_{k}$, with $N_{R O I_{k}}$ representing the number of voxels labeled as region $R O I_{k}$ in the aparc+aseg image, and $\mathrm{Val}_{i}$ represents the intensity of voxel $i$ in the PET scan.

The SUV of the whole cerebellum, consisting of 4 subcortical regions (left/right cerebellum white matter and left/right cerebellum cortex), was then calculated using (2), accounting for the varying sizes of the subregions. The SUV of the global cortical was computed in the same way, i.e., volume-weighted mean of all 68 cortical ROIs as expressed in (2.2).

$$
S U V_{C B}=\frac{S U V_{S R_{1}} \times V_{S R_{1}}+S U V_{S R_{2}} \times V_{S R_{2}}+\cdots+S U V_{S R_{N}} \times V_{S R_{N}}}{V_{S R_{1}}+V_{S R_{2}}+\cdots+V_{S R_{N}}}
$$

where $\mathrm{CB}$ represents the combined region of $\mathrm{N}$ ROIs (4 and 68 for the cerebellum and global cortical, respectively, with $S U V_{S R i}$ representing the SUV of $R O I_{i}$, and $V_{S R i}$ represents the volume of $R O I_{i}$.

Finally, each regional, as well as global SUV was normalized by the SUV of the whole cerebellum to get the regional and global AV45 standardized uptake value ratio (rSUVR and gSUVR). Such SUVR measure was then used to represent the A $\beta$ load in our analysis. 


\subsubsection{Feature Extraction Model}

To make the extracted image features match the information of the EMCI diagnosis as much as possible, and to control the number of features at a reasonable level, we proposed the ROI-based feature extraction model shown in Fig. 2. For each region, one feature was extracted based on its performance related to the EMCI phenotype, i.e., the image feature values were predicted by base learners trained on raw neuroimaging morphological variables and amyloid load.

Specifically, for each ROI, the corresponding image feature was defined as the predictive probability of being EMCI. The random forest algorithm was considered as the classifier, i.e. the base learner, using all available morphological variables (9 and 3 for cortical and subcortical regions, respectively), regional amyloid load (rSUVR) along with the global information, such as ICV and global amyloid load (gSUVR), as predictors, and the diagnosis group as output.

Random forest is an ensemble machine learning algorithm for classification and regression. It is a combination of multiple simple decision trees, and use the mode of the classes or mean prediction as the output for the classification and regression, respectively $[50]$. 


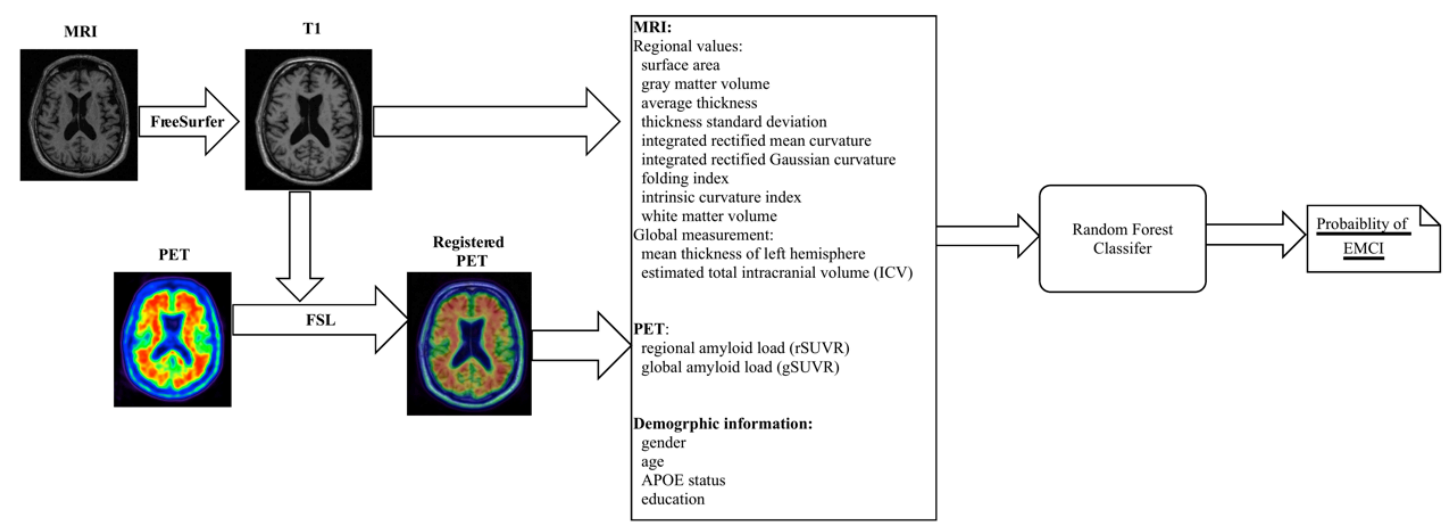

Fig. 2. Region-based neuroimang feature extraction model illustration for left entorhinal ROI in the CN vs. EMCI classification.

\subsubsection{Classification Model}

The classification model follows the stack ensemble classification model framework. Here, we used the 10-fold stacking pipeline. Specifically, the 2-level classification framework consists of the following major steps:

1. Split the training data in 10 sets: train_1, tain_2, $\ldots$, and train_10;

2. For each ROI, train the individual base leaner model, i.e. random forest, on $\left\{\right.$ train_1, taint $22, \ldots$ train $\left.\_9\right\}$, and create predictions, i.e., the probability of being EMCI for train_10;

3. Repeat step 2 nine times, i.e., train the same models on $\left\{\right.$ train $\_1, \ldots$, train ${ }_{-} \mathrm{i}-1$, train $\mathrm{i}+1$, train $\left.\_10\right\}$ and create predictions for train $\_\mathrm{i}, \mathrm{i}=2,3, \ldots 10$;

4. Rank the ROIs by the accuracy, filter out ones with accuracy less than the 1st quartile; 
5. Train the top layer classifier, i.e., SVM (polynomial kernel) model, on the remaining predictions of the base learner models that has been make on the training data in step2 and step 3 along with patients' demographic information;

6. For validation, first train the random forest models on the entire training data per ROI, and create predictions for the test data;

7. Finally, predict using the SVM (polynomial kernel) with the predictions of the base learner models that has been make for test data.

To avoid over-fitting of the ensemble model, the out-of-bag predictions for the training data were used as the predictors in step 5 to train the SVM classifier.

The proposed classification model is evaluated using 10-fold cross-validation. Specifically, we randomly divided the dataset into 10 parts and repeated the aforementioned classification framework 10 times. At each time, nine of the 10 subsets were used as training data in the aforementioned classification framework, while the remaining one were used as the testing dataset.

Once the predictions of all 10 subset are created, the true positive (TP), true negative (TN), false positive (FP), false negative (FN) can be counted. In the context of CN vs. EMCI classification, those terms means the number of subjects correctly diagnosed as EMCI, the number of subjects correctly diagnosed as $\mathrm{CN}$, the number of $\mathrm{CN}$ subjects misdiagnosed as EMCI, and the number of EMCI subjects who are diagnosed as $\mathrm{CN}$, respectively. The performance of the proposed classification model are measured by the 
accuracy (ACC), sensitivity (SEN), and specificity (SPE) which are defined as $(\mathrm{TP}+\mathrm{TN}) /(\mathrm{TP}+\mathrm{TN}+\mathrm{FN}+\mathrm{FP}), \mathrm{TP} /(\mathrm{TP}+\mathrm{FN})$, and $\mathrm{TN} /(\mathrm{FP}+\mathrm{TN})$, respectively. For each binary classification, the more serious stage are labeled as positive class, for example, $\mathrm{EMCI}$ is the positive class for the $\mathrm{CN}$ vs. EMCI classification, while $\mathrm{LMCI}$ is the positive class for the EMCI vs. LMCI classification.

\subsection{Results and Discussion}

\subsubsection{Performance of the proposed and raw imaging features}

Fig. 3 illustrates the ROC curves of the regional SUVR normalized by gSUVR, cortical thickness, regional amyloid load, gray matter volume normalized by ICV, white matter volume normlalized by ICV, global amyloid load (gSUVR), and the proposed imaging feature for the left entorhinal. The results show that the proposed imaging feature outperforms other single imaging biomarkers with the area under the curve (AUC) value of 73.2, indicating that it dose fuse the complementary information provided by different types of morphological and functional imaging measurements.

\subsubsection{Compare the baseline classification with proposed ensemble classification model}

To validate the proposed feature extraction model, we also run the SVM (Polynomial kernel) on the original imaging features as the baseline. Features do not show significant difference between $\mathrm{CN}$ and $\mathrm{EMCI}$ groups by $\mathrm{T}$ test, were filtered out, i.e., only features with uncorrected p-value less than 0.05 are considered as the predictors in the baseline 
model. In addition, we tested the independent and combined performance of the MMSE score by training the classifiers using single MMSE and combination MMSE with other imaging features, respectively. The accuracy, sensitivity, and specificity of those models are listed in Table 5. Compared with the baseline model, the proposed method effectively improved the diagnosis of EMCI from $\mathrm{CN}(\mathrm{ACC}=72.5 \%$ / 69.4 for proposed/baseline $)$, LMCI $(\mathrm{ACC}=72.3 \% / 71.9 \%)$, and $\mathrm{AD}(\mathrm{ACC}=87.6 \% / 87.4 \%)$. Such patterns were enhanced when including the MMSE score as additional predictor.

MMSE score is one of the key assess measurements of the diagnosis of $\mathrm{AD}$, patients with AD normally have MMSE score less than 24 , while for the $\mathrm{CN}$ subjects, the range is around 26 to 30. As expected, using MMSE score only, we can diagnosis AD from $\mathrm{CN}$, EMCI, or LMCI with the accuracy of $96.3 \%, 90.7 \%$, and $86.8 \%$, respectively. However, MMSE is insensitive for the early stage of $\mathrm{AD}$, especially for the EMCI stage. The $\mathrm{CN}$ vs. EMCI, CN vs. LMCI, and EMCI vs. LMCI models only have accuracy of $62.5 \%$, $68.6 \%$, and $63.9 \%$, respectively. When combing the MMSE with our proposed features, the accuracy of $\mathrm{CN}$ vs. EMCI and EMCI vs. AD reached $75.3 \%$ and $94 \%, 3 \%$ and $6.4 \%$ higher than the models using proposed features only, while accuracy of EMCI vs. LMCI is decreased to $71.3 \%$.

\subsubsection{Comparison with other feature extraction techniques}

The obtained results are comparable with or better than previously proposed approaches, especially for the more challenging classification problems: CN vs. EMCI $(72.3 \%$ / 75.3\% with/without MMSE), EMCI vs. LMCI (72.3\% / 71.3\%), and LMCI vs. AD 
(70.7\% / 84.3\%). For instance, Guerrero et al., Prasad et al., and Tripathi et al. reported CN vs. EMCI accuracy rates of $65 \%, 59.2 \%$, and $75.4 \%$, respectively. Prasad et al., Shakeri et al., and Tripathi et al. stated EMCI vs. LMCI accuracy values of $63.4 \%$, $63.0 \%$, and $71.0 \%$ repectively. Our model also outperforms on the EMCI vs. AD classification, with the accuary of $87.6 \% / 94.0 \%$, compared with other models proposed by Shakeri et al. (81.0\%) and Tripathi et al. (86.2\%) (Table 6).

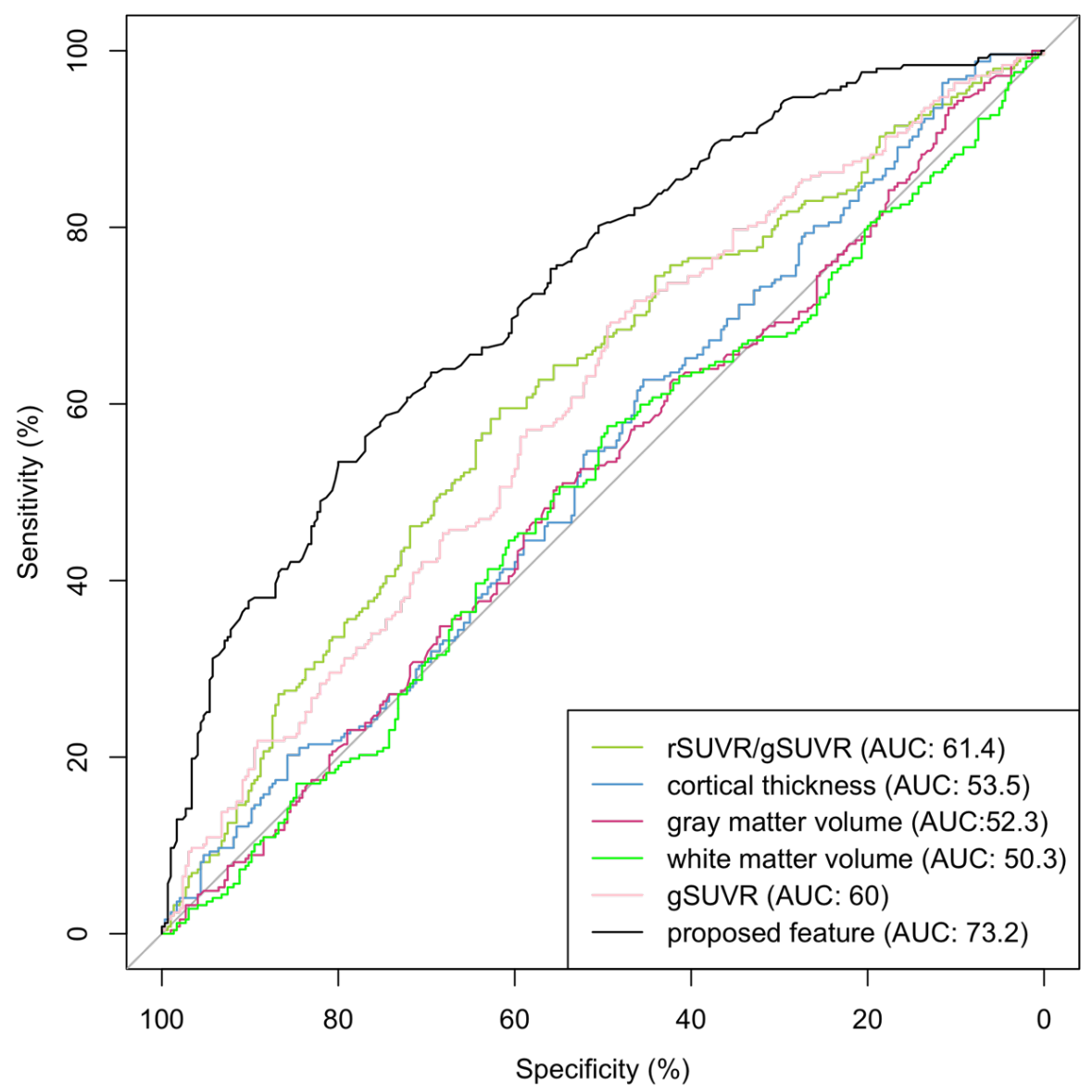

Fig. 3. ROC curves of different imaging biomarkers and the proposed imaging feature of the left entorhinal ROI for the CN vs. EMCI classification. 
Table 5. Comparison of the classification accuracy (ACC), sensitivity (SEN), and specificity (SPE) (\%) values of baseline with proposed feature extraction based model for 6 different pairs of binary

\begin{tabular}{|c|c|c|c|c|c|c|c|c|c|}
\hline & \multicolumn{3}{|c|}{ CN vs. EMCI } & \multicolumn{3}{|c|}{ CN vs. LMCI } & \multicolumn{3}{|c|}{ CN vs. AD } \\
\hline & ACC & SEN & SPE & ACC & SEN & SPE & ACC & SEN & SPE \\
\hline baseline & 69.4 & 69.4 & 69.4 & 76.6 & 77.1 & 76.3 & 92.6 & 91.0 & 93.6 \\
\hline proposed & 72.5 & 73.0 & 71.9 & 76.4 & 79.9 & 74.6 & 91.1 & 88.6 & 92.7 \\
\hline MMSE & 62.5 & 63.2 & 61.3 & 68.6 & 67.5 & 69.3 & 96.3 & 91.8 & 99.6 \\
\hline Baseline+MMSE & 70.5 & 70.0 & 71.2 & 77.3 & 77.2 & 77.3 & 94.3 & 95.3 & 93.8 \\
\hline \multirow[t]{3}{*}{ Propsed+MMSE } & 75.3 & 75.2 & 75.3 & 77.5 & 78.3 & 77.0 & 96.5 & 96.2 & 96.8 \\
\hline & \multicolumn{3}{|c|}{ EMCI vs. LMCI } & \multicolumn{3}{|c|}{ EMCI vs. AD } & \multicolumn{3}{|c|}{ LMCI vs. AD } \\
\hline & ACC & SEN & SPE & ACC & SEN & SPE & ACC & SEN & SPE \\
\hline baseline & 71.9 & 73.0 & 71.6 & 87.4 & 83.9 & 89.1 & 74.1 & 71.3 & 76.3 \\
\hline proposed & 72.3 & 73.8 & 71.9 & 87.6 & 87.0 & 87.9 & 70.7 & 71.7 & 70.1 \\
\hline MMSE & 63.9 & 65.5 & 63.7 & 90.7 & 79.3 & 99.6 & 85.8 & 76.7 & 98.0 \\
\hline Baseline+MMSE & 72.7 & 73.8 & 72.4 & 90.7 & 88.7 & 91.7 & 80.6 & 78.5 & 82.4 \\
\hline Propsed+MMSE & 71.3 & 71.2 & 71.3 & 94.0 & 92.8 & 94.7 & 84.3 & 84.1 & 84.5 \\
\hline
\end{tabular}

Table 6. Comparison of the classification accuracy (ACC), sensitivity (SEN), and specificity (SPE) (\%) values of the proposed model with others for 6 different pairs of binary classification

\begin{tabular}{|c|c|c|c|c|c|c|c|c|c|}
\hline & \multicolumn{3}{|c|}{ CN vs. EMCI } & \multicolumn{3}{|c|}{ CN vs. LMCI } & \multicolumn{3}{|c|}{$\mathrm{CN}$ vs. $\mathrm{AD}$} \\
\hline & ACC & SEN & SPE & $\mathrm{ACC}$ & SEN & SPE & $\mathrm{ACC}$ & SEN & SPE \\
\hline Guerrero et al. (2014) & 65 & 61 & 69 & - & - & - & 86 & 86 & 85 \\
\hline Prasad et al. (2014) & 59.2 & - & - & 62.8 & 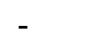 & - & 78.2 & . & - \\
\hline Shakeri et al. (2016) & 56 & 52 & 60 & 59 & 52 & 65 & 84 & 73 & 89 \\
\hline Tripathi et al. (2017) & 75.4 & 74.0 & 76.6 & 72.6 & 71.37 & 74.3 & 88.7 & 83.1 & 91.5 \\
\hline \multirow[t]{3}{*}{ Propsed+MMSE } & 75.3 & 75.2 & 75.3 & 77.5 & 78.3 & 77.0 & 96.5 & 96.2 & 96.8 \\
\hline & \multicolumn{3}{|c|}{ EMCI vs. LMCI } & \multicolumn{3}{|c|}{ EMCI vs. AD } & \multicolumn{3}{|c|}{ LMCI vs. AD } \\
\hline & ACC & SEN & SPE & ACC & SEN & SPE & ACC & SEN & SPE \\
\hline Guerrero et al. (2014) & - & - & - & - & - & - & - & - & - \\
\hline Prasad et al. (2014) & 63.4 & - & - & - & - & - & - & - & - \\
\hline Shakeri et al. (2016) & 63 & 62 & 66 & 81 & 70 & 82 & 67 & 58 & 73 \\
\hline Tripathi et al. (2017) & 71.0 & 75.6 & 65.5 & 86.2 & 83.2 & 88.2 & 76.8 & 79.8 & 74.2 \\
\hline Propsed+MMSE & 71.3 & 71.2 & 71.3 & 94.0 & 92.8 & 94.7 & 84.3 & 84.1 & 84.5 \\
\hline
\end{tabular}

\subsection{Conclusion}

We have proposed an ensemble framework based on region-based neuroimaging feature extraction model for the classification of Alzheimer's Disease. The features were extracted as the probability of being disease form the base learner trained on all regional morphological variables provided by FreeSurfer, regional amyloid load, and the global 
information such as ICV and global amyloid load. Experimental evaluation on the ADNI dataset demonstrates the effectiveness of our approach especially in classifying CN vs. EMCI, EMCI vs. LMCI, and EMCI vs. AD, indicating that the proposed features preserved the sensitivity to change in a single brain region especially at the very early stage of the disease and might be used as the potential effective imaging biomarker especially for the EMCI diagnosis and clinical study. 


\section{CHAPTER III}

\section{REGIONAL IMAGE FEATURES MODEL FOR THE GENOME-WIDE ASSOCIATION STUDY OF ALZHEIMER'S DISEASE}

\subsection{Goal}

Compared to the traditional disease phenotypes (with or without disease), we can obtain closer association or even better insight by introducing intermediate phenotypes, which are often continuous values, [51-54]. Recently, brain imaging is commonly considered as a source of intermediate phenotypes that augment our understanding of the subtle and complex relationship between genetics and disease phenotypes, which is often termed as imaging genetics. The imaging genetics takes into consideration the fact that certain image-based features can serve as promising brain phenotypes for discovering the disease related genes.

One of the main challenges in the area of imaging genetics is the extremely high dimensionality of image and genetic data (millions of voxels or SNPs), albeit with a relatively small number of subjects in $\mathrm{AD}$ research (few thousands). To overcome this challenge, some researchers proposed algorithms that consider only few image features such as the intensities of selected voxels $[55,56]$, regional cortical atrophy $[18,57]$, and brain activation [58]; or only few SNPs using univariate methods, i.e., performing a standard statistical test on each pair of a candidate SNP and related imaging features [5759]. To control the false positive rate, multiple comparisons adjustment is needed [60]. This may reduce the power of the test and hence overlook the weaker SNPs that jointly 
create an additive effect. To overcome such shortcomings, multivariate techniques, such as parse reduced rank regression (sRRR) [56], sparse Partial Least Squares (sPLS) [61] and Canonical Correlation Analysis (CCA) [62] have been introduced in the more recent literature. The sPLS performs better compared to others [62]. However, these methods do not consider the diagnosis information in their model, and thus the detected SNPs may not be immediately related to AD.

In this study, we propose a regional image feature extraction model for obtaining image features associated with the disease, and then use sPLS regression model to detect the SNPs associated with these extracted image features. Thus, instead of modeling a direct link between genetic variants and disease label, we captured disease information indirectly.

\subsection{Material}

\subsubsection{Study Participants}

For this particular study, given its set objectives, $103 \mathrm{AD}$ patients and 139 normal controls $(\mathrm{CN})$ form ADNI1 with available whole genome imputed genotypes information was considered. All individuals underwent a 1.5 Tesla T1-weighted MRI scan with a cognition assessment at baseline. The demographic characteristics of the participants were shown in Table 7. 
Table 7. Participants demographic and clinical information for the imaging genetic study

\begin{tabular}{llll}
\hline Characteristics & CN $(139)$ & AD $(103)$ & p-value $^{\mathrm{a}}$ \\
\hline Female/Male & $62 / 77$ & $49 / 54$ & 0.7431 \\
Age & $76.2(4.9)^{\mathrm{b}}$ & $75.1(7.6)$ & 0.1558 \\
Education & $16.2(2.6)$ & $14.8(3.2)$ & $\mathbf{0 . 0 0 0 3}$ \\
MMSE & $29.17(1.02)$ & $23.54(1.95)$ & $\mathbf{0 . 0 0 0 0}$ \\
\hline
\end{tabular}

${ }^{a}$ Significant group differences ( $\mathrm{T}$ test for continuous and Chi-square test for categorical values, significance level is 0.05 by default)

${ }^{\mathrm{b}}$ Values are represented as mean(standard deviation)

\subsubsection{Image Processing}

Each MRI image was processed sequentially by the FreeSurfer (Version 5.3.0) (http://freesurfer.net, [63]), Advanced Normalization Tools (ANTs) (http://picsl.upenn.edu/software/ants/, [64]) and the Mindboggle software (http://www.mindboggle.info/, [65]). Firstly, the FreeSurfer was used to transform the original MRI to the standard MNI 305 space, followed by skull-stripping, segmenting, and delineating cortical and subcortical regions, and to generate thereafter labeled cortical surfaces, and labeled cortical and neocortical volumes. The ANTs software was then used to perform the brain volume extraction, segmentation, and registration-based labeling. Finally, the Mindboggle software package was executed to automatically analyze the shape of anatomical labels and extract features from outputs of FreeSurfer and ANTs.

Ultimately, 43 measures of each of the 50 neocortical brain regions (ROI) are obtained as indicated in Table 8 . Those 50 ROIs include caudal anterior cingulate, caudal middle frontal, cuneus, entorhinal, fusiform, inferior parietal, inferior temporal, lateral occipital, lateral orbitofrontal, lingual, medial orbitofrontal, middle temporal, parahippocampal, 
paracentral, pars opercularis, pericalcarine, postcentral, precentral, precuneus, superior frontal, superiorparietal, superior temporal, supramarginal, transverse temporal, and insula on both left and right hemispheres.

Table 8 . The 43 Statistical summaries for each region

\begin{tabular}{ll}
\hline Measure Type & Number of statistical measures \\
\hline Surface Area & 1 \\
Thickness & 8 - median, MAD, mean, SD, skew, kurtosis, $.25^{\mathrm{a}}, .75^{\mathrm{b}}$ \\
Travel depth & 8 - median, MAD, mean, SD, skew, kurtosis, .25, .75 \\
Geodesic depth & 8 - median, MAD, mean, SD, skew, kurtosis, .25, .75 \\
Convexity & 8 - median, MAD, mean, SD, skew, kurtosis, .25, .75 \\
Mean curvature & 8 - median, MAD, mean, SD, skew, kurtosis, .25,.75 \\
Thickness.thickinthehead & 1 \\
Volume & 1 \\
\hline${ }^{a}$ first quartile of the corresponding measure type \\
\end{tabular}

\subsubsection{Genetic Data Processing}

The samples were genotyped on the Illumina Human610-Quad BeadChip or the Illumina HumanOmniExpress BeadChip. Considering the variety of SNP genotyping platforms, genetic data were imputed to a common reference set. The pre-impute quality control (QC) threshold and methods applied were as follows: All genome-wide association studies (GWAS) data are mapped to hg19/b37 positions and flipped to plus strand; the minor allele frequency $(\mathrm{MAF})<0.05$; SNP Call Rate $<0.98$; SAMPLE Call Rate $<0.98$; Hardy-Weinberg equilibrium $(\mathrm{HWE})<0.001$. Then, QC'd Batch 1 and 2 genotypes were pre-phased prior to SNP imputation using SHAPEITv2. Furthermore, the SNP imputation was performed using IMPUTE2. Overall, 12,568,804 SNPs were provided by ADNI. 


\subsection{Method}

\subsubsection{ROI-based feature extraction}

To make the extracted image features match the information of the $\mathrm{AD}$ diagnosis as much as possible, and to control the number of features at a reasonable level, we proposed the ROI-based feature extraction model shown in Fig. 4. For each region, one feature was extracted based on its performance related to the AD phenotype, i.e., the image feature values were predicted by base learners trained on raw neuroimaging morphological variables (Table 8).

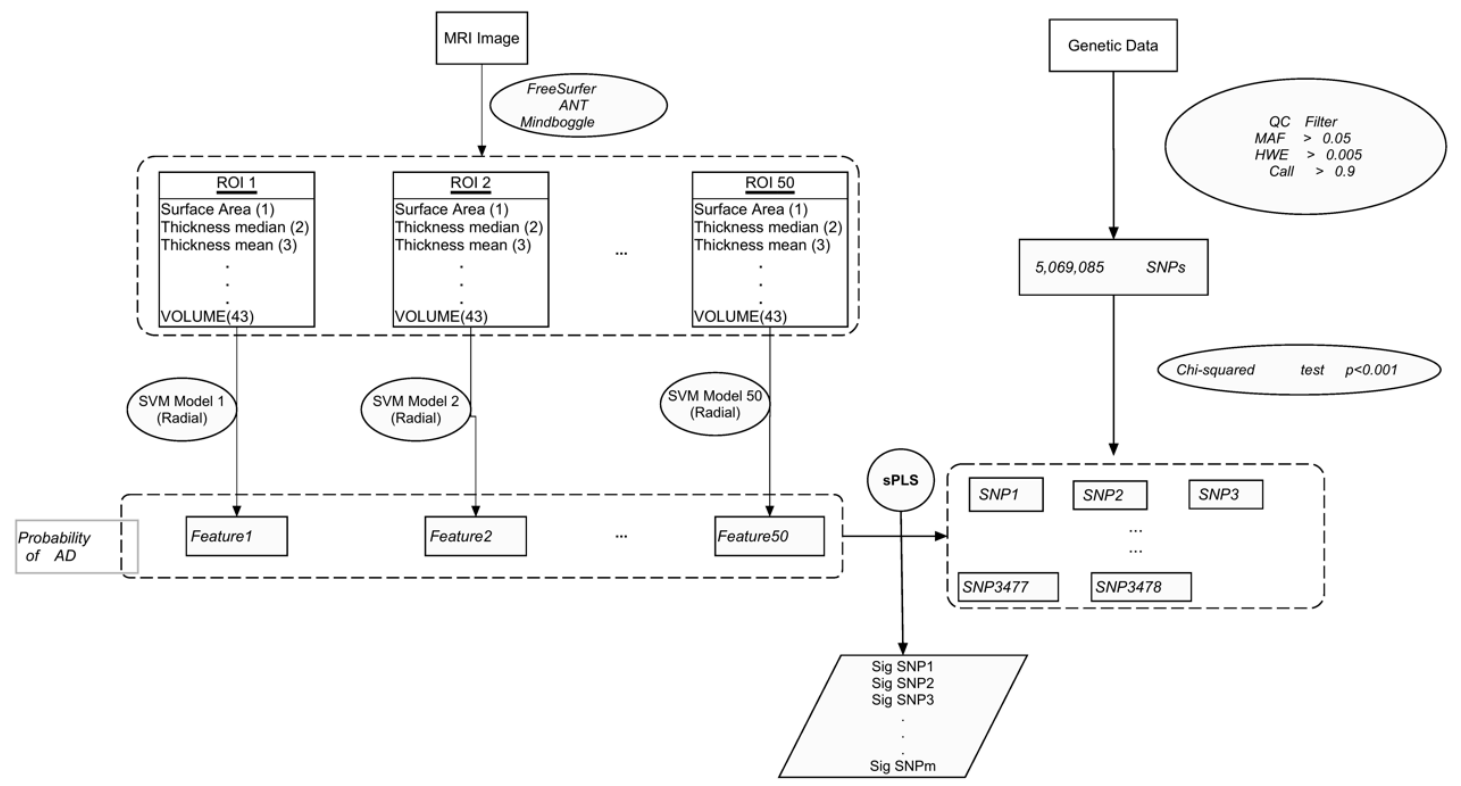

Fig. 4. The flowchart illustrating the genome-wide association study using proposed ROI-based feature extraction model

Specifically, for each ROI, the corresponding image feature was defined as the predictive probability of being AD. The Support Vector Machine (SVM) with a Radial Kernel model [66] was considered as the classifier, i.e. the base learner, using all 43 
measurements as predictors, and the diagnosis group as output. After tuning the parameters based on the 10-fold cross-validation accuracy performance, we predicted each subject using the final model, and set the prediction probability of AD as the image feature of that specific ROI. Overall, 50 image features were extracted and were used as the brain phenotypes in the following regression model to detect the SNPs of interest.

\subsubsection{SNPs Selection}

To reduce the dimension of the genetic data, we first eliminated SNPs that do not satisfy the following $\mathrm{QC}$ criteria: $\mathrm{MAF} \geq 0.05$; an $\mathrm{HWE}$ dose not showing significant difference at level 0.005; and with the SNP call rate $\geq 0.9$. Chi-squared test was then conducted for each remaining SNP. Only SNPs that show significant difference between CN and AD group were considered in the following study, i.e. uncorrected p-value $<0.001$. After filtering, 3478 out of the total $12,568,804$ SNPs were finally selected. The count of minor alleles $(0,1$, or 2$)$ was used to represent the SNP.

\subsubsection{Sparse partial least squares regression}

The partial least squares regression (PLS) is based on the latent decomposition and could handle multicollinearity. It is considered to model the associations between two sets of variables via a small number of unobserved latent variables (LV), which are linear combinations of the original variables. Assume there are $\mathrm{K}$ hidden components, the PLS regression model builds successive and orthogonal LV for each set such that at each step the covariance between the pair of LVs is maximal (3.1). 


$$
\max _{\left|u_{k}\right| \|\left|v_{k}\right|=1} \operatorname{cov}\left(X_{k-1} u_{k}, Y_{k-1} v_{k}\right)
$$

where k represents the k-th step to get the k-th hidden components. Elements $U_{k}$ and $V_{k}$ define the weight vectors for the linear combinations of the variables of sets $X_{k-1}$ and $Y_{k-1}$, respectively. $X_{k-1}$ and $Y_{k-1}$ are the residuals of sets $\mathrm{X}$ and $\mathrm{Y}$ after their regression on the k-1 pervious LVs (3.2).

$$
\begin{gathered}
X_{k-2}=T P^{t}+X_{k-1} \\
Y_{k-2}=T Q^{t}+Y_{k-1}
\end{gathered}
$$

the $\mathrm{T}$ matrix produces the $\mathrm{K}$ linear combinations, $\mathrm{P}$ and $\mathrm{Q}$ are matrices of coefficients, and $X_{k-1}$ and $Y_{k-1}$ are matrices of residuals. Considering that the LVs of the PLS have contributions from all the predictors, it is hard to make adequate interpretations in the presence of a large number of noise variables. Chun and Keles [67] proposed the sparse partial least squares regression model expressed in (3.3) by imposing additional L1 penalization to (3.1),

$$
\min _{\| u_{k}||\left|v_{k}\right|=1}-\operatorname{cov}\left(X_{k-1} u_{k}, Y_{k-1} v_{k}\right)+\lambda_{X}\|u\|_{1}+\lambda_{Y}\|v\|
$$

where $\lambda_{X}$ and $\lambda_{Y}$ are the L1-penalization parameters for the weight vectors of $\mathrm{X}$ and $\mathrm{Y}$, respectively. In the context of this study, X consists of the 3478 selected SNPs after filtering, and $\mathrm{Y}$ includes the 50 extracted image features. Based on the 10 -fold crossvalidated mean-squared prediction error, the optimal model with parameters $\mathrm{K}=2$ and $\lambda_{X}=\lambda_{Y}=0.9$ was employed. 


\subsection{Results}

\subsubsection{The Feature Extraction Model Evaluation}

The principal components analysis (PCA) was performed on the 50 extracted features, and the first two principal components (PC1 and PC2) separated the $\mathrm{CN}$ and $\mathrm{AD}$ subjects

clearly, which means that those extracted features do capture the expected disease information (Fig. 5a). The feature that contributes most to the PC1 is extracted from the left inferior temporal, and for the PC2, it is from the right precentral. Fig. 5b shows the plot of those two features. Fig. 6 shows the accuracy obtained from 50 final SVM models. The top 5 ROIs entorhinal, fusiform, middle temporal, inferior temporal, and parahippocampal are all $\mathrm{AD}$ vulnerable regions [68], which provides credence to the method for extracting base learner derived features.

\subsubsection{Genetic association results}

The sPLS models finally detected 15 significant SNPs (Table 9). 14 SNPs are located on chromosome 19 and have been reported as correlated with AD [69-71]. Among them, rs769449 (APOE4), rs429358 (APOE), rs73052335 (APOC1) rs12972970 (PVRL2), rs6857 (PVRL2), $\quad$ rs2075650 (TOMM40), rs34404554 (TOMM40), $\quad$ rs11556505 (TOMM40) are frequently reported as strongly correlated with $\mathrm{AD}$ and all are on chromosome $19[18,19,21,55,72]$. The rs917100 located on chromosome 8 was also detected. The performance of those 15 SNPs in terms of the coefficient estimate of all 50 image-based features was shown in the heatmap given in Fig 7. 

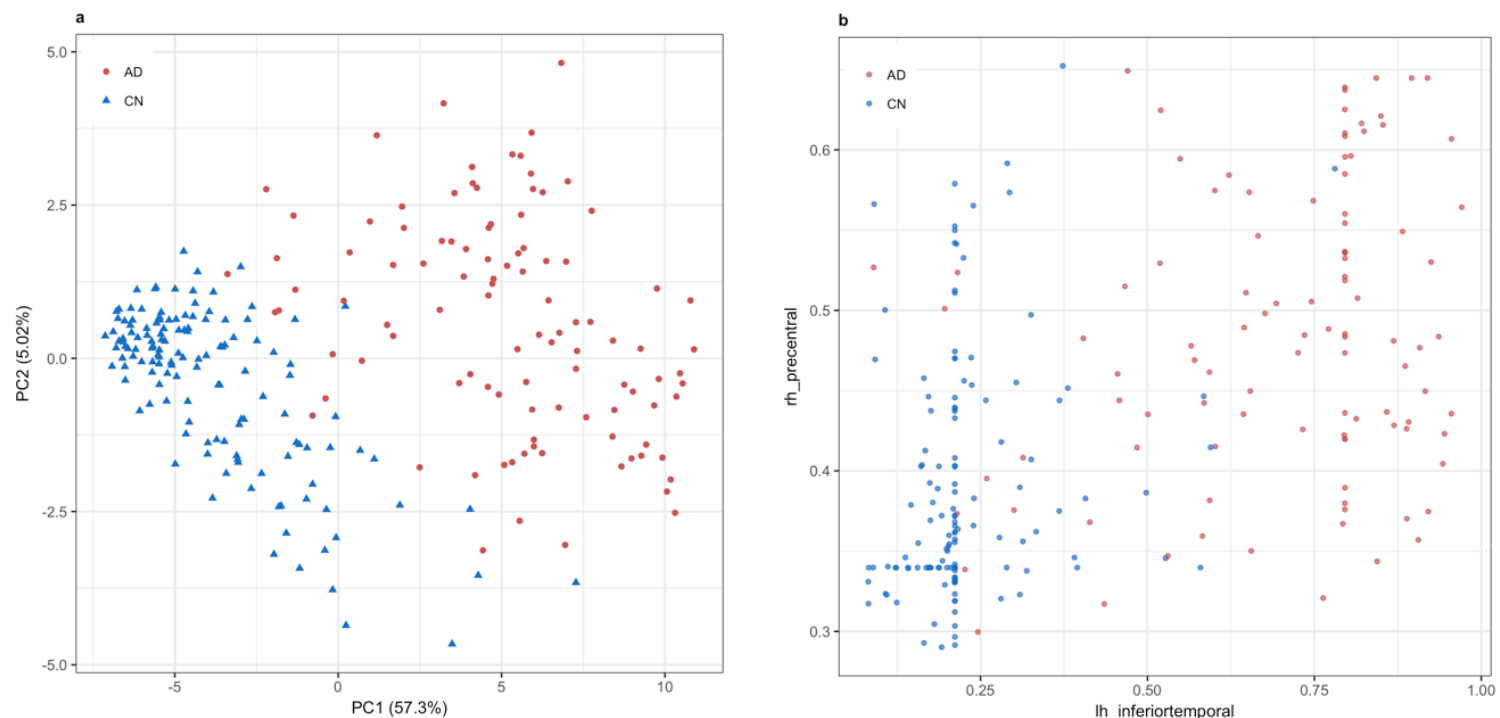

Fig. 5. Principal Component Analysis. a The PCA map of extracted image features for $\mathrm{CN}$ and $\mathrm{AD}$ groups. $\mathrm{x}$-axis is the fist principal components (PC1) and y-axis is the second principal components (PC2); b Image features plot. Features extracted from the left inferior temporal and the right precentral contributes most for the $\mathrm{PC} 1$ and $\mathrm{PC} 2$ respectively.

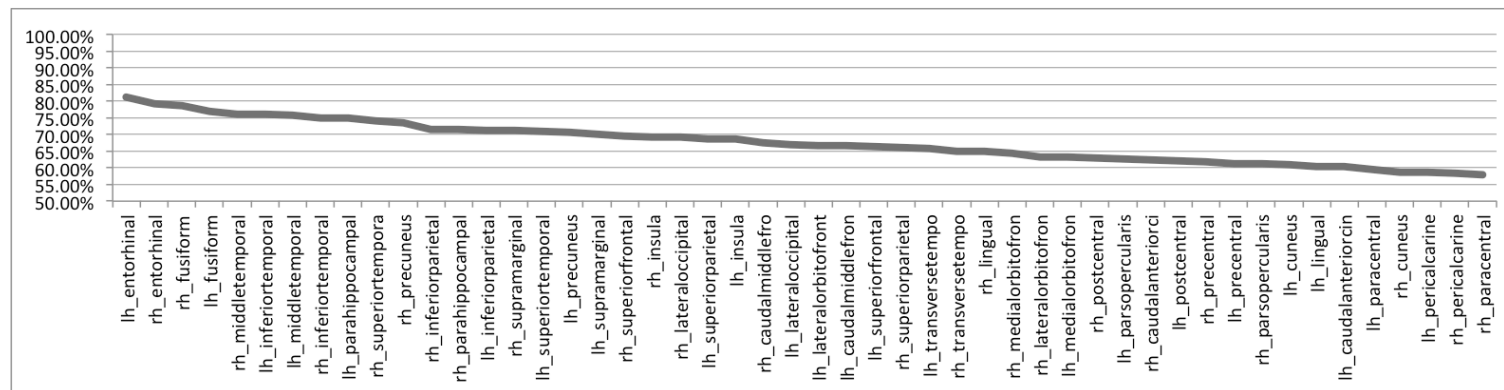

Fig. 6. The accuracy of 50 final SVM(Radial) Models (10-fold cross validation). One model for each brain region.

Concerning the relationship to ROI-based features, rs12972970 (PVRL2), rs12972156, rs34342646, rs71352238, rs2075650 (TOMM40), rs34404554 (TOMM40), rs11556505 (TOMM40), and rs142042446 show very similar patterns, those SNPs also contribute negatively for both the first and second LV in the sPLS regression model (Table 9). While rs769449 (APOE4), rs429358 (APOE), rs73052335 (APOC1), rs10414043 (APOE 
(distance=3063), APOC1 (distance=2208)), and rs7256200 (APOE (distance=3285), APOC1(distance=1986)) have stronger correlation with some AD vulnerable regions, such as entorhinal, middle temporal and precuneus than the aforementioned SNPs, and they contribute negatively for the first LV but positively for the second LV. The rs917100 show similar pattern with APOE and APOC1 genes in the contribution to the LVs, but has stronger associations with the image features.

Table 9. Fifteen SNPs Selected by sPLS model

\begin{tabular}{llllll}
\hline SNP & Chromosome & Position & Gene & Comp1 & Comp2 \\
\hline rs769449 & 19 & 45410002 & APOE & -0.00499 & 0.00255 \\
rs73052335 & 19 & 45420082 & APOC1 & -0.00495 & 0.00537 \\
rs10414043 & 19 & 45415713 & APOE(dist=3063),APOC1(dist=2208) & -0.00489 & 0.00384 \\
rs7256200 & 19 & 45415935 & APOE(dist=3285),APOC1(dist=1986) & -0.00489 & 0.00384 \\
rs12972156 & 19 & 45387459 & & -0.00481 & -0.00286 \\
rs12972970 & 19 & 45387596 & PVRL2 & -0.00481 & -0.00286 \\
rs34342646 & 19 & 45388130 & & -0.00481 & -0.00286 \\
rs71352238 & 19 & 45394336 & & -0.00481 & -0.00286 \\
rs2075650 & 19 & 45395619 & TOMM40 & -0.00481 & -0.00286 \\
rs34404554 & 19 & 45395909 & TOMM40 & -0.00481 & -0.00286 \\
rs11556505 & 19 & 45396144 & TOMM40 & -0.00481 & -0.00286 \\
rs429358 & 19 & 45411941 & APOE & -0.00480 & 0.00438 \\
rs142042446 & 19 & 45386467 & & -0.00470 & -0.00446 \\
rs6857 & 19 & 45392254 & PVRL2 & -0.00468 & 0.00065 \\
rs917100 & 8 & 5591150 & & -0.00418 & 0.06374 \\
\hline
\end{tabular}

${ }^{a}$ The weights for each latent variable in the sPLS model. 


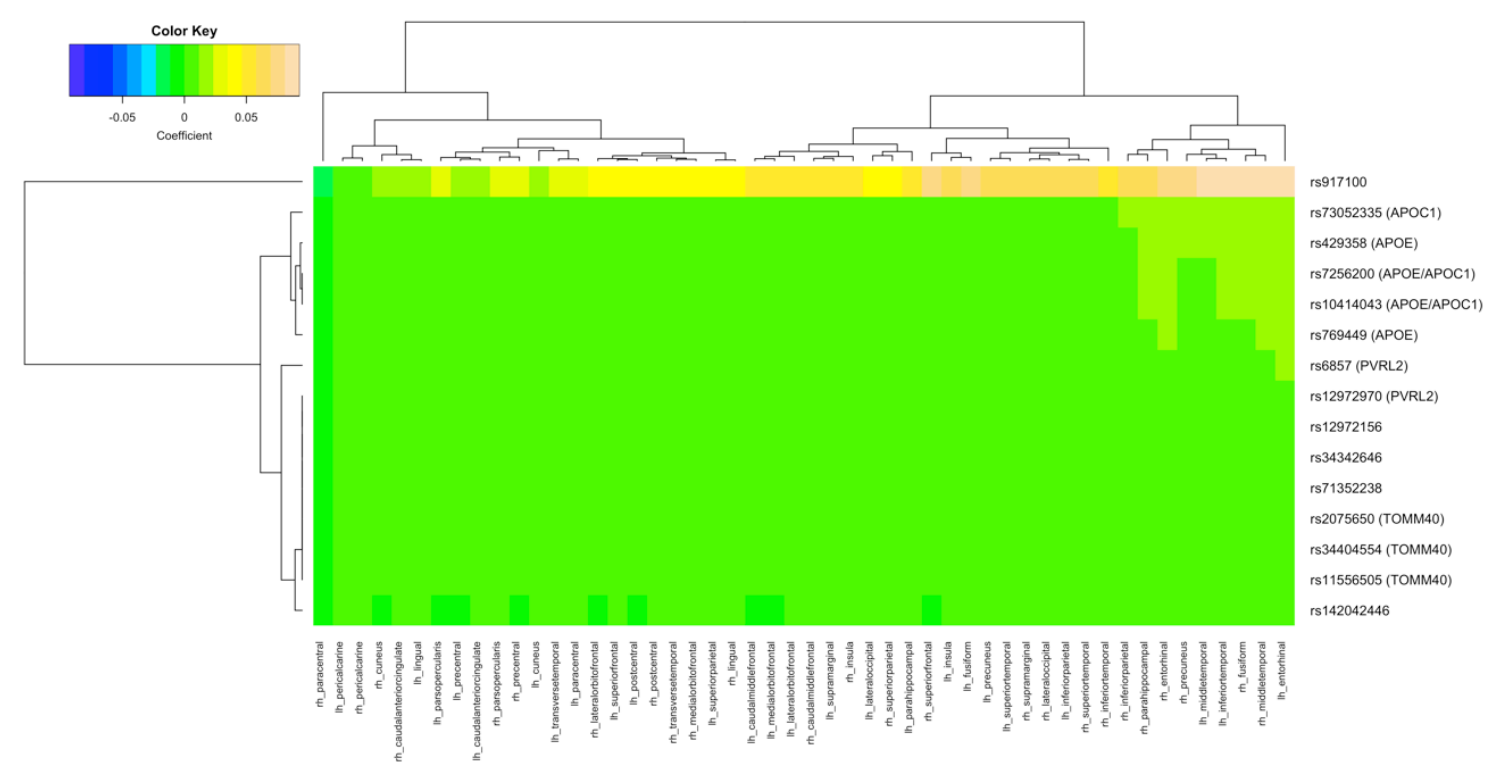

Fig. 7. Heat map of the selected 15 SNPs by sPLS using extracted image features as responses.

\subsection{Conclusion}

We proposed and demonstrated an imaging feature extraction model and applied it on the whole ADNI genetic dataset to identify genetic variants associated with AD. The associations between imaging and AD were captured using SVM base learner for each brain region. The AD related genetic variants were then selected via the sPLS regression model. The proposed model can be widely employed to other GWAS study of neurological diseases. On the other hand, besides the volume and cortical thickness, the proposed imaging feature extraction model considers more detailed information of the brain regions and could be used as additional predictors to enhance the diagnosis model of $\mathrm{AD}$, and to further explore the performance of brain regions on the progression of $\mathrm{AD}$ as well. 


\section{CHAPTER IV}

\section{THE RELATIONSHIP OF BRAIN AMYLOID LOAD AND APOE STATUS TO REGIONAL CORTICAL THINING AND COGNITION}

\subsection{Goal}

The relationship between APOE4 positive status $(\mathrm{E} 4+)$ and higher $\mathrm{A} \beta$ load is well known, as is the relationship of both E4+ status and higher A $\beta$ load to a greater risk for developing Alzheimer's disease and a greater degree of neurodegeneration [23, 73-75]. However, the relationship of APOE4 status to neurodegeneration and cognitive decline, independent of $\mathrm{A} \beta$ load, and the relationship of $\mathrm{A} \beta$ load to neurodegeneration and cognitive decline, independent of APOE4 status, are not known and to our knowledge have not been studied thus far.

In the present study, we examined both the combined and independent associations between global A $\beta$ load, APOE4 status and regional coritcal thickness (CTh) among four different diagnostic groups in the ADNI sample, including cognitively normal $(\mathrm{CN})$, early mild cognitive impairment (EMCI), late mild cognitive impairment (LMCI) or AD dementia participants. A unique aspect of this investigation was to determine the independent and combined effects of APOE4 status and A $\beta$ load on regional $\mathrm{CTh}$ and cognition among individuals presenting with minimal (EMCI) or no overt memory impairment $(\mathrm{CN})$. 


\subsection{Materials and Methods}

\subsubsection{Data}

Data used in the preparation of this article were obtained from the ADNI database (adni.loni.usc.edu). Evaluations included: (1) Medical and neurological evaluation and routine labs; (2) Clinical Dementia Rating (CDR) scale, which was used as the index of functional ability [76]; (3) neuropsychological assessment, including the ADAS Cog 13 (ADAS13) and Mini-Mental State Exam (MMSE) subtests, used as the global index of cognitive ability [77], and the Rey Auditory Verbal Learning Test (RAVLT) and subtests, used as the index of memory performance [78]; (4) volumetric MRI; and (5) ${ }^{18} \mathrm{~F}-\mathrm{AV} 45$ amyloid PET scans. The time gap between MRI and PET scans was less than 3 months. A blood sample for assessment of APOE genotype was also obtained.

Baseline demographic, clinical and APOE data were compared for 906 subjects, diagnosed as CN (251), EMCI (297), LMCI (196), or AD (162), as shown in Table 10. Subjects with one or more APOE4 alleles, i.e. APOE4 carriers, were classified as E4+, while those with no APOE4 alleles, i.e. APOE4 non-carrier, were classified as E4-.

\subsubsection{Image Processing}

Image processing pipeline is same as descripted in Chapter II. In this study, regional CTh in $\mathrm{AD}$ signature regions, previously identified by several groups $[79,80]$ was evaluated. These include the entorhinal cortex (ERC), parahippocampal gyrus (PHG), inferior temporal gyrus (ITG), temporal pole (TP), medial orbital frontal gyrus, superior temporal 
gyrus, rostral middle frontal gyrus, inferior parietal lobule (including angular gyrus), superior parietal lobule, supramarginal gyrus, precuneus, posterior cingulate gyrus and the mean CTh based on the aforementioned regions.

In terms of AV45 PET, only global amyloid load was considered. A widely used threshold value of 1.10 is used here to delineate $A \beta$ positive $(A m y+$, SUVR $>1.10)$ and A $\beta$ negative (Amy-, SUVR $<=1.10$ ) status $[81,82]$.

Table 10. Participant clinical information

\begin{tabular}{|c|c|c|c|c|c|}
\hline & $\begin{array}{c}\text { CN } \\
(n=251)\end{array}$ & $\begin{array}{c}\text { EMCI } \\
(n=297)\end{array}$ & $\begin{array}{c}\text { LMCI } \\
(\mathrm{n}=196)\end{array}$ & $\begin{array}{c}\text { AD } \\
(\mathrm{n}=162)\end{array}$ & F value \\
\hline Female/Male & $128 / 123$ & $132 / 165$ & $85 / 111$ & $68 / 94$ & $4.36^{\mathrm{b}}$ \\
\hline E4-/E4+ & $184 / 67$ & $169 / 128$ & $92 / 104$ & $54 / 108$ & $70.32 * * * c$ \\
\hline Age & $75.5(6.5)^{\mathrm{a}}$ & $71.5(7.4)$ & $73.8(8.1)$ & $74.9(7.8)$ & $14.85^{* * *}$ \\
\hline EDUCATION & $16.4(2.6)$ & $16.0(2.7)$ & $16.3(2.7)$ & $15.8(2.7)$ & $2.71 *$ \\
\hline \multicolumn{6}{|c|}{ Type of Cognitive Test } \\
\hline CDRSB & $0.05(0.2)$ & $1.31(0.78)$ & $1.76(1.06)$ & $4.84(2.07)$ & $633.12 * * *$ \\
\hline ADAS13 & $9.09(4.54)$ & $12.72(5.51)$ & $17.9(7.5)$ & $31.55(8.81)$ & $434.53 * * *$ \\
\hline MMSE & $29.04(1.23)$ & $28.32(1.57)$ & $27.61(1.85)$ & $22.77(2.71)$ & $448.73 * * *$ \\
\hline RAVLT_immediate & $45.35(10.58)$ & $39.47(10.8)$ & $33.21(10.82)$ & $22.31(7.03)$ & $183.76^{* * *}$ \\
\hline RAVLT_learning & $5.74(2.44)$ & $5.29(2.45)$ & $3.92(2.58)$ & $1.91(1.77)$ & $102.04 * * *$ \\
\hline RAVLT_\% forgetting & $36.22(27.79)$ & $46.98(29.72)$ & $67.37(31.34)$ & 90.1(19.91) & $142.34 * * *$ \\
\hline \multicolumn{6}{|c|}{$\begin{array}{l}{ }^{a} \text { Values are represented as mean(sd), except gender and APOE gene status, which are frequencies } \\
\text { instead }\end{array}$} \\
\hline \multicolumn{6}{|c|}{$\begin{array}{l}{ }^{\mathrm{b}} \text { Significant group differences test (ANOVA for continuous and Chi-square test for categorical values, } \\
\text { significance level is } 0.05 \text { by default) }\end{array}$} \\
\hline
\end{tabular}

\subsubsection{Statistical Methods}

The statistical analysis was performed using R software (R 3.3.0) [83] and the default significant level was determined as 0.05 . To examine the independent effect of $A \beta$ load on regional $\mathrm{CTh}$, by controlling for the effects of APOE4 status, two-way analysis of 
covariance (ANCOVA), i.e., 4 (diagnosis: $\mathrm{CN}, \mathrm{EMCI}, \mathrm{LMCI}, \mathrm{AD})$ by 2 (A $\beta$ load status: Amy+, Amy-) ANCOVA, was conducted per region, with APOE4 status (E4+, E4-) entered as an categorical covariates.

Similarly, to investigate the individual effects of APOE4 status after controlling for global A $\beta$ load, a series of 4 (diagnosis: CN, EMCI, LMCI, AD) by 2 (APOE4 status: E4+, E4-) ANCOVAs were conducted, with global A $\beta$ load (SUVR) as an interval level covariate. As a complementary analysis, to assess the earliest individual effects of APOE4 status, a series of 2 (diagnosis: CN, EMCI) by 2 (APOE4 status: E4+, E4-) ANCOVAs were performed among 548 individuals diagnosed as $\mathrm{CN}$ or EMCI.

To examine the earliest combined effects of both APOE4 status and A $\beta$ load status on regional $\mathrm{CTh}$ and on several cognitive variables (MMSE, RAVLT sub scores, and ADAS13 score), we focused on above-mentioned CN and EMCI participants $(\mathrm{n}=548)$, and divided them into four groups: E4-/Amy- $(\mathrm{n}=241), \mathrm{E} 4-/ \mathrm{Amy}+(\mathrm{n}=112), \mathrm{E} 4+/ \mathrm{Amy}-$ $(\mathrm{n}=73)$, and $\mathrm{E} 4+/ \mathrm{Amy}+(\mathrm{n}=122)$. A series of one-way ANCOVAs were conducted, using age as a covariate, and with CTh or cognitive scores as the dependent variables.

Multiple comparison correction was performed in all aforementioned ANCOVA analyses to control the false discovery rate (FDR). Statistically significant results (FDR-adjusted $p$ value $<0.05$ ) were further examined using post hoc Tukey HSD test. 


\subsection{Results}

E4+ status was associated with higher $A \beta$ load across all diagnostic groups, as shown in Table 11 and Fig. 8.

\subsubsection{Effect of A $\beta$ Load Status on CTh after Controlling for APOE4 Status}

Results for the left hemisphere only are reported, since findings in both the left and right hemispheres were similar. As can be seen in Table 12 and Fig. 9, there was a statistically significant main effect for A $\beta$ load, accounting for the effect of APOE4 status, on CTh, for all brain regions examined, with the exception of the temporal pole (TP), superior frontal gyrus and rostral middle frontal gyrus. The most significant effects were noted in the inferior temporal gyrus (ITG) $(\mathrm{F}=31.18$, diff (Amy+ - Amy-) $=-0.099)$, inferior parietal lobule $(F=20.82$, diff $=-0.068)$, precuneus $(F=16.55$, diff $=-0.058)$, the mean CTh for all regions $(F=15.6$, diff $=-0.046)$, entorhinal cortex $(E R C)(F=14.59$, diff $=-$ 0.159), and supramarginal gyrus $(\mathrm{F}=10.98$, diff $=-0.051)$. In all these regions Amy+ status was associated with reduced CTh, adjusting for the effects of APOE4 status.

Table 11. Effect of APOE4 status on global amyloid load (SUVR) in different diagnostic groups

\begin{tabular}{lcccc}
\hline & E4- & E4+ & t test $^{\mathrm{a}}$ & \multicolumn{1}{c}{ P value $^{-3.65649}$} \\
\hline CN & $1.079(0.16)$ & $1.178(0.2)$ & -0.0021 \\
EMCI & $1.115(0.18)$ & $1.246(0.21)$ & -5.76863 & 0 \\
LMCI & $1.147(0.23)$ & $1.368(0.21)$ & -7.12632 & 0 \\
AD & $1.271(0.26)$ & $1.439(0.16)$ & -4.32684 & 0.00002 \\
\hline
\end{tabular}

${ }^{\mathrm{a}} \mathrm{H}_{0}: \operatorname{SUVR}(\mathrm{E} 4-)=\operatorname{SUVR}(\mathrm{E} 4+)$ vs $\mathrm{H}_{1}: \operatorname{SUVR}(E 4-)<\operatorname{SUVR}(E 4+)$ 


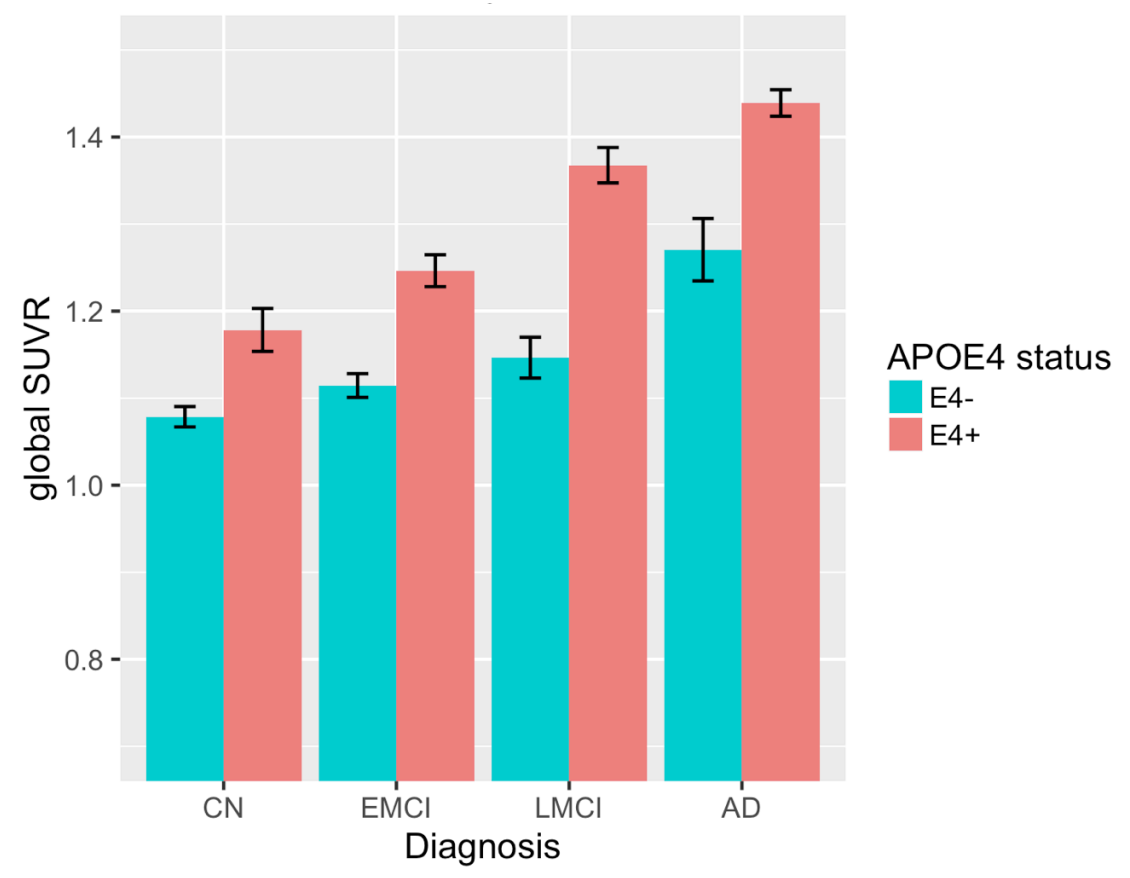

Fig. 8. Differences in mean amyloid load $\left({ }^{18} \mathrm{~F}-\mathrm{AV} 45\right)$ SUVR for each diagnostic group between E4- and E4+ participants. The error bar on the barplot represents the standard error value.

Table 12 also shows a strong main effect for diagnosis. Using post hoc tests (Tukey HSD), it was found that CTh was reduced among AD participants compared to the three other diagnostic groups, and also among LMCI participants, as compared to EMCI and CN participants. This pattern appeared to hold for every brain region included in these analyses, with the exception of the superior parietal lobule, where EMCI participants had greater CTh than the other diagnostic groups, which did not statistically differ from each other. With rare exceptions, CTh was equivalent between $\mathrm{CN}$ and EMCI participants. In the supramarginal gyrus and precuneus, CTh was greater in EMCI than in $\mathrm{CN}$ participants. Statistically significant interaction terms (diagnosis with A $\beta$ load status) were observed for the ITG and inferior parietal lobule, in which Amy+ status was associated with reduced $\mathrm{CTh}$ only in the $\mathrm{LMCI}$ and the $\mathrm{AD}$ stages. 
Table 12. Effect of amyloid status on regional CTh, independent of APOE4 status (left hemisphere)

\begin{tabular}{|c|c|c|c|c|c|c|c|c|}
\hline & $\begin{array}{c}\mathrm{CN} \\
\text { Amy- } 165 \\
\text { Amy+ } 86\end{array}$ & $\begin{array}{c}\text { EMCI } \\
\text { Amy- } 149 \\
\text { Amy+ } 148\end{array}$ & $\begin{array}{c}\text { LMCI } \\
\text { Amy- } 66 \\
\text { Amy+ } 130\end{array}$ & $\begin{array}{c}\text { AD } \\
\text { Amy- } 19 \\
\text { Amy+ } 143\end{array}$ & $\begin{array}{c}\mathrm{F}^{\mathrm{a}} \\
\text { Amyloid }\end{array}$ & $\begin{array}{c}\mathrm{F}^{\mathrm{a}} \\
\text { Diagnosis }\end{array}$ & $\begin{array}{c}\text { post_hoc } \\
\text { Tukey } \\
\text { (Diagnosis) }\end{array}$ & $\begin{array}{c}\mathrm{F}^{\mathrm{a}} \\
\text { Diagnosis } \\
\text { by } \\
\text { Amyloid }\end{array}$ \\
\hline Age & $\begin{array}{l}74.51(6.8)^{b} \\
76.75(5.7)\end{array}$ & $\begin{array}{l}69.19(7.4) \\
73.46(6.8)\end{array}$ & $\begin{array}{l}73.68(9.4) \\
73.61(7.3)\end{array}$ & $\begin{array}{l}77.49(8.2) \\
74.38(7.7)\end{array}$ & $10.64 * *^{\mathrm{c}}$ & $15.79 * * *$ & $\begin{array}{c}\mathrm{EMCI}< \\
\text { All }\end{array}$ & $4.78^{* *}$ \\
\hline Entorhinal & $\begin{array}{l}3.34(0.38) \\
3.27(0.37)\end{array}$ & $\begin{array}{l}3.32(0.46) \\
3.26(0.43)\end{array}$ & $\begin{array}{l}3.17(0.52) \\
2.96(0.52)\end{array}$ & $\begin{array}{l}2.77(0.74) \\
2.58(0.49)\end{array}$ & $14.59 * * *$ & $42.18 * * *$ & $\begin{array}{c}\mathrm{AD}<\mathrm{All} \\
\mathrm{LMCI}< \\
\mathrm{EMCI}, \mathrm{CN}\end{array}$ & 1.56 \\
\hline $\begin{array}{c}\text { Parahippoc } \\
\text { ampal }\end{array}$ & $\begin{array}{l}2.67(0.34) \\
2.58(0.41)\end{array}$ & $\begin{array}{l}2.66(0.36) \\
2.67(0.31)\end{array}$ & $\begin{array}{c}2.52(0.39) \\
2.5(0.4)\end{array}$ & $\begin{array}{l}2.44(0.39) \\
2.31(0.35)\end{array}$ & $6.74 * *$ & $15.6^{* * *}$ & $\begin{array}{c}\mathrm{AD}< \\
\text { EMCI, CN } \\
\text { LMCI }< \\
\text { EMCI, CN }\end{array}$ & 1.23 \\
\hline $\begin{array}{l}\text { Inferior } \\
\text { temporal }\end{array}$ & $\begin{array}{l}2.66(0.16) \\
2.61(0.16)\end{array}$ & $\begin{array}{l}2.66(0.18) \\
2.61(0.19)\end{array}$ & $\begin{array}{l}2.61(0.18) \\
2.57(0.23)\end{array}$ & $\begin{array}{l}2.57(0.26) \\
2.39(0.24)\end{array}$ & $31.18^{* * *}$ & $13.86^{* * *}$ & $\begin{array}{c}\mathrm{AD}<\mathrm{All} \\
\mathrm{LMCI}< \\
\mathrm{EMCI}, \mathrm{CN}\end{array}$ & $3.13^{*}$ \\
\hline $\begin{array}{l}\text { Temporal } \\
\text { pole }\end{array}$ & $\begin{array}{l}3.56(0.33) \\
3.53(0.36)\end{array}$ & $\begin{array}{c}3.48(0.37) \\
3.5(0.34)\end{array}$ & $\begin{array}{c}3.5(0.35) \\
3.35(0.45)\end{array}$ & $\begin{array}{l}3.08(0.63) \\
3.11(0.51)\end{array}$ & 2.46 & $23.19 * * *$ & $\begin{array}{c}\mathrm{AD}<\mathrm{All} \\
\mathrm{LMCI}< \\
\mathrm{CN}\end{array}$ & 1.99 \\
\hline $\begin{array}{l}\text { Medial } \\
\text { orbitofront } \\
\text { al }\end{array}$ & $\begin{array}{l}2.28(0.15) \\
2.27(0.18)\end{array}$ & $\begin{array}{l}2.27(0.14) \\
2.23(0.16)\end{array}$ & $\begin{array}{l}2.23(0.17) \\
2.22(0.17)\end{array}$ & $\begin{array}{c}2.25(0.2) \\
2.19(0.16)\end{array}$ & $8.14 * *$ & $4.57 * *$ & $\begin{array}{c}\mathrm{AD}<\mathrm{CN} \\
\mathrm{LMCI}< \\
\mathrm{CN}\end{array}$ & 1.3 \\
\hline $\begin{array}{l}\text { Superior } \\
\text { frontal }\end{array}$ & $\begin{array}{l}2.48(0.17) \\
2.46(0.17)\end{array}$ & $\begin{array}{l}2.52(0.15) \\
2.49(0.15)\end{array}$ & $\begin{array}{l}2.45(0.17) \\
2.42(0.16)\end{array}$ & $\begin{array}{l}2.37(0.17) \\
2.36(0.18)\end{array}$ & 3.79 . & $15.17 * * *$ & $\begin{array}{c}\mathrm{AD}<\mathrm{All} \\
\mathrm{LMCI}< \\
\mathrm{EMCI}\end{array}$ & 0.14 \\
\hline $\begin{array}{l}\text { Rostral } \\
\text { Middle } \\
\text { Frontal }\end{array}$ & $\begin{array}{l}2.15(0.14) \\
2.15(0.15)\end{array}$ & $\begin{array}{l}2.18(0.13) \\
2.15(0.12)\end{array}$ & $\begin{array}{l}2.14(0.14) \\
2.11(0.13)\end{array}$ & $\begin{array}{l}2.09(0.16) \\
2.07(0.15)\end{array}$ & 1.87 & $8.2^{* * *}$ & $\begin{array}{c}\mathrm{AD}< \\
\text { EMCI, CN } \\
\text { LMCI }< \\
\text { EMCI }\end{array}$ & 0.64 \\
\hline $\begin{array}{c}\text { Superior } \\
\text { Parietal }\end{array}$ & $\begin{array}{l}1.96(0.17) \\
1.96(0.17)\end{array}$ & $\begin{array}{l}2.01(0.14) \\
2.01(0.14)\end{array}$ & $\begin{array}{l}1.97(0.16) \\
1.94(0.16)\end{array}$ & $\begin{array}{l}1.95(0.12) \\
1.86(0.18)\end{array}$ & $6.08 *$ & $10.02 * * *$ & $\begin{array}{c}\text { EMCI }> \\
\text { All }\end{array}$ & 1.69 \\
\hline $\begin{array}{l}\text { Inferior } \\
\text { Parietal }\end{array}$ & $\begin{array}{l}2.19(0.16) \\
2.18(0.17)\end{array}$ & $\begin{array}{l}2.23(0.14) \\
2.21(0.15)\end{array}$ & $\begin{array}{c}2.2(0.14) \\
2.13(0.16)\end{array}$ & $\begin{array}{c}2.18(0.18) \\
2.02(0.2)\end{array}$ & $20.82 * * *$ & $10.67 * * *$ & $\begin{array}{c}\mathrm{AD}<\mathrm{All} ; \\
\mathrm{LMCI}< \\
\mathrm{EMCI}\end{array}$ & $4.78^{* *}$ \\
\hline $\begin{array}{l}\text { Supramarg } \\
\text { inal }\end{array}$ & $\begin{array}{l}2.31(0.18) \\
2.31(0.18)\end{array}$ & $\begin{array}{l}2.37(0.15) \\
2.34(0.16)\end{array}$ & $\begin{array}{c}2.3(0.17) \\
2.26(0.17)\end{array}$ & $\begin{array}{l}2.26(0.19) \\
2.16(0.18)\end{array}$ & $10.98 * * *$ & $16.51 * * *$ & $\begin{array}{c}\mathrm{AD}<\mathrm{All} \\
\text { EMCI }> \\
\text { LMCI, CN }\end{array}$ & 1.71 \\
\hline Precuneus & $\begin{array}{l}2.14(0.17) \\
2.12(0.17)\end{array}$ & $\begin{array}{l}2.19(0.14) \\
2.15(0.15)\end{array}$ & $\begin{array}{c}2.14(0.16) \\
2.1(0.15)\end{array}$ & $\begin{array}{l}2.11(0.14) \\
1.99(0.18)\end{array}$ & $16.55^{* * *}$ & $10.33 * * *$ & $\begin{array}{c}\mathrm{AD}<\mathrm{All} \\
\text { EMCI }> \\
\text { LMCI, CN }\end{array}$ & 1.92 \\
\hline $\begin{array}{l}\text { Posterior } \\
\text { Cingulate }\end{array}$ & $\begin{array}{c}2.38(0.18) \\
2.4(0.18)\end{array}$ & $\begin{array}{l}2.41(0.17) \\
2.39(0.17)\end{array}$ & $\begin{array}{c}2.4(0.17) \\
2.37(0.17)\end{array}$ & $\begin{array}{l}2.39(0.22) \\
2.31(0.18)\end{array}$ & $4.25^{*}$ & 1.7 & & 1.66 \\
\hline $\begin{array}{c}\text { Mean } \\
\text { CTh_left }\end{array}$ & $\begin{array}{l}2.30(0.13) \\
2.29(0.14)\end{array}$ & $\begin{array}{l}2.33(0.11) \\
2.31(0.12)\end{array}$ & $\begin{array}{l}2.28(0.13) \\
2.24(0.13)\end{array}$ & $\begin{array}{l}2.23(0.15) \\
2.15(0.14)\end{array}$ & $15.6^{* * *}$ & $20.62 * * *$ & $\begin{array}{c}\mathrm{AD}<\mathrm{All} \\
\mathrm{LMCI}< \\
\mathrm{EMCI}, \mathrm{CN}\end{array}$ & 1.54 \\
\hline
\end{tabular}

${ }^{\mathrm{a}} \mathrm{F}$ value is adjusted for APOE4 Status (4 X 2 ANCOVA test)

${ }^{\mathrm{b}}$ Values are represented as mean(SD), upper is for Amy- group and lower is for Amy + group

${ }^{\mathrm{c}} \mathrm{p}<.1 ;{ }^{*} \mathrm{p}<.05 ; * * \mathrm{p}<.01 ; * * * \mathrm{p}<.001$ 

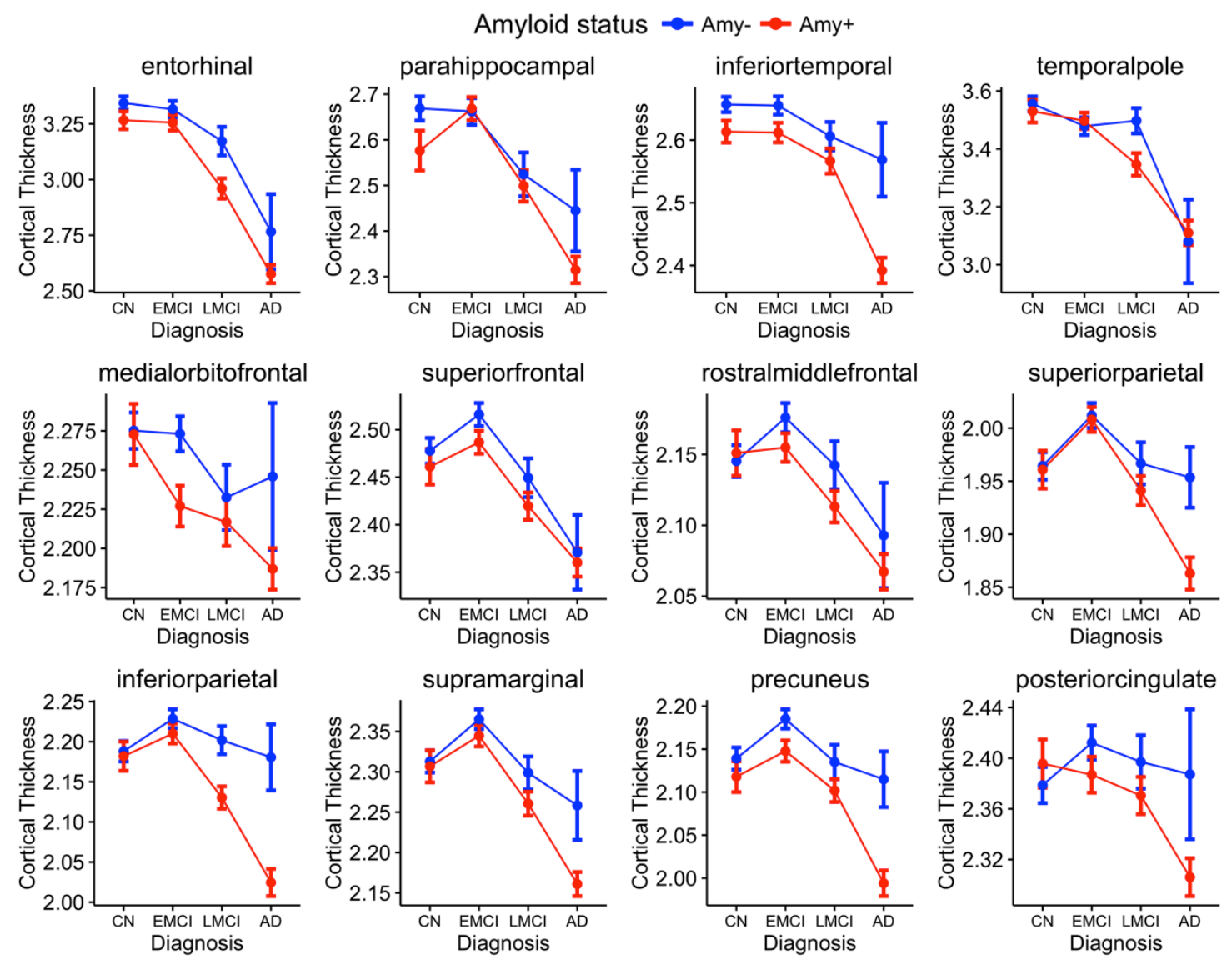

Fig. 9. Barplot of CTh among Amy- and Amy+ participants in 4 diagnostic groups for 12 brain regions.

\subsubsection{Effect of APOE4 Status on CTh after Controlling for Aß Load}

From Table 13, it can be seen that the diagnostic group effect was similar to the pattern observed in Table 11, i.e., AD patients had less CTh than the other study groups and that LMCI participants had less CTh than EMCI and CN participants, while CTh was equivalent between $\mathrm{CN}$ and EMCI participants. An inspection of the regional $\mathrm{CTh}$ by APOE4 status in Fig. 10 shows that for most brain regions analyzed, CTh among $\mathrm{CN}$ and EMCI participants is numerically greater among those who are E4+ than E4-. However, the reverse is generally true among LMCI and particularly AD participants, for whom 
CTh is generally lower among E4+ than E4- participants. After adjusting for global A $\beta$ load (SUVR), there was a main effect for APOE4 status only in the ITG (diff(E4+ - E4-) $=0.048 \mathrm{~mm}, \mathrm{~F}=9.99, \mathrm{p}=0.0016)$ and medial orbitofrontal gyrus $(\operatorname{diff}=0.027 \mathrm{~mm}, \mathrm{~F}=$ 4.83, $\mathrm{p}=0.028)$, in which it can be observed that $\mathrm{CTh}$ was overall greater among E4+ than among E4- participants (Fig. 10). Furthermore, the interaction term in Table 13 shows significant difference only in the ERC, where the CTh is greater only among $\mathrm{CN}$ and EMCI participants who are E4+ as compared to those who are E4-, whereas among LMCI and $\mathrm{AD}$ participants, $\mathrm{CTh}$ is greater among E4- as compared to $\mathrm{E} 4+$ participants (Fig. 10).

More importantly, when CN and EMCI subjects were analyzed independently (Table 14), E4+ status (controlling for A $\beta$ load) was associated with increased CTh in the ERC (diff $=0.123 \mathrm{~mm}, \mathrm{~F}=9.68, \mathrm{p}=0.002)$, PHG $($ diff $=0.082 \mathrm{~mm}, \mathrm{~F}=6.02, \mathrm{p}=0.014)$, ITG (diff $=0.059 \mathrm{~mm}, \mathrm{~F}=12.56, \mathrm{p}=0.0004)$, and TP $(\operatorname{diff}=0.091 \mathrm{~mm}, \mathrm{~F}=7.47, \mathrm{p}=0.006)$. 
Table 13. Effect of APOE4 status on regional CTh, independent of A $\beta$ load (left hemisphere)

\begin{tabular}{|c|c|c|c|c|c|c|c|c|}
\hline & $\begin{array}{c}\mathbf{C N} \\
\text { E4- } 184 \\
\text { E4+ } 67\end{array}$ & $\begin{array}{c}\text { EMCI } \\
\text { E4- } 169 \\
\text { E4+ } 128\end{array}$ & $\begin{array}{c}\text { LMCI } \\
\text { E4- } 92 \\
\text { E4+ } 104\end{array}$ & $\begin{array}{c}\text { AD } \\
\text { E4- } 54 \\
\text { E4+ } 108\end{array}$ & $\begin{array}{c}\mathbf{F}^{\mathrm{a}} \\
\text { APOE4 } \\
\text { Status }\end{array}$ & $\begin{array}{c}\mathbf{F}^{\mathrm{a}} \\
\text { Diagnosis }\end{array}$ & $\begin{array}{c}\text { post_hoc_Tuke } \\
\text { y (Diagnosis) }\end{array}$ & $\begin{array}{c}\mathbf{F}^{\mathbf{a}} \\
\text { Diagnosi } \\
\text { s by } \\
\text { APOE4 } \\
\end{array}$ \\
\hline Age & $\begin{array}{l}75.77(6.3)^{b} \\
73.93(7.0)\end{array}$ & $\begin{array}{l}72.07(7.3) \\
70.33(7.5)\end{array}$ & $\begin{array}{l}74.96(8.6) \\
72.46(7.4)\end{array}$ & $\begin{array}{l}76.43(8.6) \\
73.90(7.3)\end{array}$ & $29.12 * * *$ & $12.89 * * *$ & $\mathrm{EMCI}<$ All & 0.44 \\
\hline Entorhinal & $\begin{array}{l}3.30(0.38) \\
3.35(0.37)\end{array}$ & $\begin{array}{l}3.22(0.46) \\
3.37(0.41)\end{array}$ & $\begin{array}{l}3.11(0.54) \\
2.96(0.51)\end{array}$ & $\begin{array}{l}2.69(0.60) \\
2.55(0.48)\end{array}$ & 0.02 & $68.18 * * *$ & $\begin{array}{c}\mathrm{AD}<\mathrm{All} \\
\mathrm{LMCI}<\mathrm{EMCI}, \\
\mathrm{CN}\end{array}$ & $4.84 * *$ \\
\hline $\begin{array}{l}\text { Parahippo } \\
\text { campal }\end{array}$ & $\begin{array}{l}2.62(0.38) \\
2.68(0.34)\end{array}$ & $\begin{array}{l}2.64(0.32) \\
2.70(0.34)\end{array}$ & $\begin{array}{l}2.50(0.39) \\
2.52(0.39)\end{array}$ & $\begin{array}{l}2.37(0.38) \\
2.31(0.34)\end{array}$ & 1.71 & $25.56 * * *$ & $\begin{array}{c}\mathrm{AD}<\mathrm{All} \\
\mathrm{LMCI}<\mathrm{EMCI} \\
\mathrm{CN}\end{array}$ & 1.08 \\
\hline $\begin{array}{l}\text { Inferior } \\
\text { temporal }\end{array}$ & $\begin{array}{l}2.63(0.15) \\
2.68(0.17)\end{array}$ & $\begin{array}{l}2.62(0.19) \\
2.65(0.19)\end{array}$ & $\begin{array}{l}2.57(0.21) \\
2.59(0.22)\end{array}$ & $\begin{array}{l}2.42(0.27) \\
2.41(0.24)\end{array}$ & $9.99 * *$ & $32.41 * * *$ & $\begin{array}{c}\mathrm{AD}<\mathrm{All} \\
\mathrm{LMCI}<\mathrm{CN}\end{array}$ & 0.5 \\
\hline $\begin{array}{l}\text { Temporal } \\
\text { pole }\end{array}$ & $\begin{array}{l}3.53(0.35) \\
3.60(0.32)\end{array}$ & $\begin{array}{l}3.45(0.37) \\
3.53(0.34)\end{array}$ & $\begin{array}{l}3.42(0.40) \\
3.38(0.44)\end{array}$ & $\begin{array}{l}3.11(0.53) \\
3.10(0.52)\end{array}$ & 1.55 & $32.87 * * *$ & $\begin{array}{c}\mathrm{AD}<\mathrm{All} \\
\mathrm{LMCI}<\mathrm{CN}\end{array}$ & 1.1 \\
\hline $\begin{array}{l}\text { Medial } \\
\text { orbitofron } \\
\text { tal }\end{array}$ & $\begin{array}{l}2.27(0.15) \\
2.28(0.18)\end{array}$ & $\begin{array}{l}2.25(0.15) \\
2.25(0.15)\end{array}$ & $\begin{array}{l}2.22(0.16) \\
2.22(0.18)\end{array}$ & $\begin{array}{l}2.17(0.18) \\
2.21(0.15)\end{array}$ & $4.83 *$ & $4.78 * *$ & $\begin{array}{c}\mathrm{AD}<\mathrm{EMCI}, \\
\mathrm{CN}\end{array}$ & 0.53 \\
\hline $\begin{array}{l}\text { Superior } \\
\text { frontal }\end{array}$ & $\begin{array}{l}2.46(0.18) \\
2.50(0.16)\end{array}$ & $\begin{array}{l}2.51(0.14) \\
2.50(0.16)\end{array}$ & $\begin{array}{l}2.44(0.17) \\
2.42(0.16)\end{array}$ & $\begin{array}{l}2.33(0.15) \\
2.37(0.19)\end{array}$ & 2.01 & $22.2 * * *$ & $\begin{aligned} \mathrm{AD} & <\mathrm{All} ; \\
\mathrm{LMCI} & <\mathrm{EMCI}\end{aligned}$ & 1.49 \\
\hline $\begin{array}{l}\text { Rostral } \\
\text { Middle } \\
\text { Frontal }\end{array}$ & $\begin{array}{l}2.14(0.15) \\
2.15(0.14)\end{array}$ & $\begin{array}{l}2.18(0.12) \\
2.15(0.13)\end{array}$ & $\begin{array}{l}2.13(0.14) \\
2.11(0.12)\end{array}$ & $\begin{array}{l}2.05(0.16) \\
2.08(0.15)\end{array}$ & 0 & $14.73 * * *$ & $\begin{aligned} \mathrm{AD} & <\mathrm{All} ; \\
\mathrm{LMCI} & <\mathrm{EMCI}\end{aligned}$ & 1.8 \\
\hline $\begin{array}{l}\text { Superior } \\
\text { Parietal }\end{array}$ & $\begin{array}{l}1.95(0.16) \\
2.00(0.17)\end{array}$ & $\begin{array}{l}2.02(0.14) \\
2.00(0.14)\end{array}$ & $\begin{array}{l}1.96(0.16) \\
1.94(0.16)\end{array}$ & $\begin{array}{l}1.88(0.18) \\
1.87(0.18)\end{array}$ & 0.57 & $18.66 * * *$ & $\begin{array}{c}\mathrm{AD}<\text { All; } \\
\mathrm{LMCI}<\mathrm{EMCI}\end{array}$ & 1.7 \\
\hline $\begin{array}{l}\text { Inferior } \\
\text { Parietal }\end{array}$ & $\begin{array}{l}2.18(0.16) \\
2.21(0.18)\end{array}$ & $\begin{array}{l}2.22(0.15) \\
2.22(0.15)\end{array}$ & $\begin{array}{l}2.18(0.16) \\
2.13(0.15)\end{array}$ & $\begin{array}{l}2.06(0.20) \\
2.03(0.21)\end{array}$ & 0.1 & $25.75 * * *$ & $\begin{aligned} \mathrm{AD} & <\mathrm{All} ; \\
\mathrm{LMCI} & <\mathrm{EMCI}\end{aligned}$ & 2.11 . \\
\hline $\begin{array}{l}\text { Supramar } \\
\text { ginal }\end{array}$ & $\begin{array}{l}2.30(0.18) \\
2.34(0.17)\end{array}$ & $\begin{array}{l}2.35(0.15) \\
2.36(0.16)\end{array}$ & $\begin{array}{l}2.29(0.17) \\
2.26(0.17)\end{array}$ & $\begin{array}{l}2.17(0.19) \\
2.17(0.18)\end{array}$ & 2.08 & $30.71 * * *$ & $\begin{array}{c}\mathrm{AD}<\mathrm{All} \\
\mathrm{LMCI}<\mathrm{EMCI}\end{array}$ & 0.99 \\
\hline Precuneus & $\begin{array}{l}2.12(0.16) \\
2.16(0.17)\end{array}$ & $\begin{array}{l}2.17(0.14) \\
2.16(0.15)\end{array}$ & $\begin{array}{l}2.12(0.16) \\
2.10(0.16)\end{array}$ & $\begin{array}{l}2.03(0.17) \\
2.00(0.18)\end{array}$ & 0.36 & $22.49 * * *$ & $\begin{aligned} \mathrm{AD} & <\mathrm{All} \\
\mathrm{LMCI} & <\mathrm{EMCI}\end{aligned}$ & 1.39 \\
\hline $\begin{array}{l}\text { Posterior } \\
\text { Cingulate }\end{array}$ & $\begin{array}{l}2.37(0.18) \\
2.42(0.18)\end{array}$ & $\begin{array}{l}2.41(0.17) \\
2.38(0.17)\end{array}$ & $\begin{array}{l}2.38(0.16) \\
2.38(0.18)\end{array}$ & $\begin{array}{l}2.33(0.20) \\
2.31(0.18)\end{array}$ & 0.16 & $5.47 * * *$ & $\mathrm{AD}<\mathrm{All}$ & 2.03 \\
\hline $\begin{array}{l}\text { MeanCTh } \\
\text { left }\end{array}$ & $\begin{array}{l}2.29(0.14) \\
2.32(0.13)\end{array}$ & $\begin{array}{l}2.32(0.11) \\
2.32(0.12)\end{array}$ & $\begin{array}{l}2.27(0.14) \\
2.25(0.13)\end{array}$ & $\begin{array}{l}2.16(0.15) \\
2.16(0.14)\end{array}$ & 2.31 & $38.84 * * *$ & $\begin{array}{c}\mathrm{AD}<\mathrm{All} \\
\mathrm{LMCI}<\mathrm{EMCI} \\
\mathrm{CN}\end{array}$ & 1.25 \\
\hline
\end{tabular}

${ }^{\mathrm{a}} \mathrm{F}$ value is adjusted for global $\mathrm{A} \beta$ load (4 X 2 ANCOVA test)

${ }^{\mathrm{b}}$ Values are represented as mean(SD), upper is for E4- group and lower is for E4+ group

${ }^{\mathrm{c}} \mathrm{p}<.1 ;{ }^{*} \mathrm{p}<.05 ; * * \mathrm{p}<.01 ; * * * \mathrm{p}<.001$ 

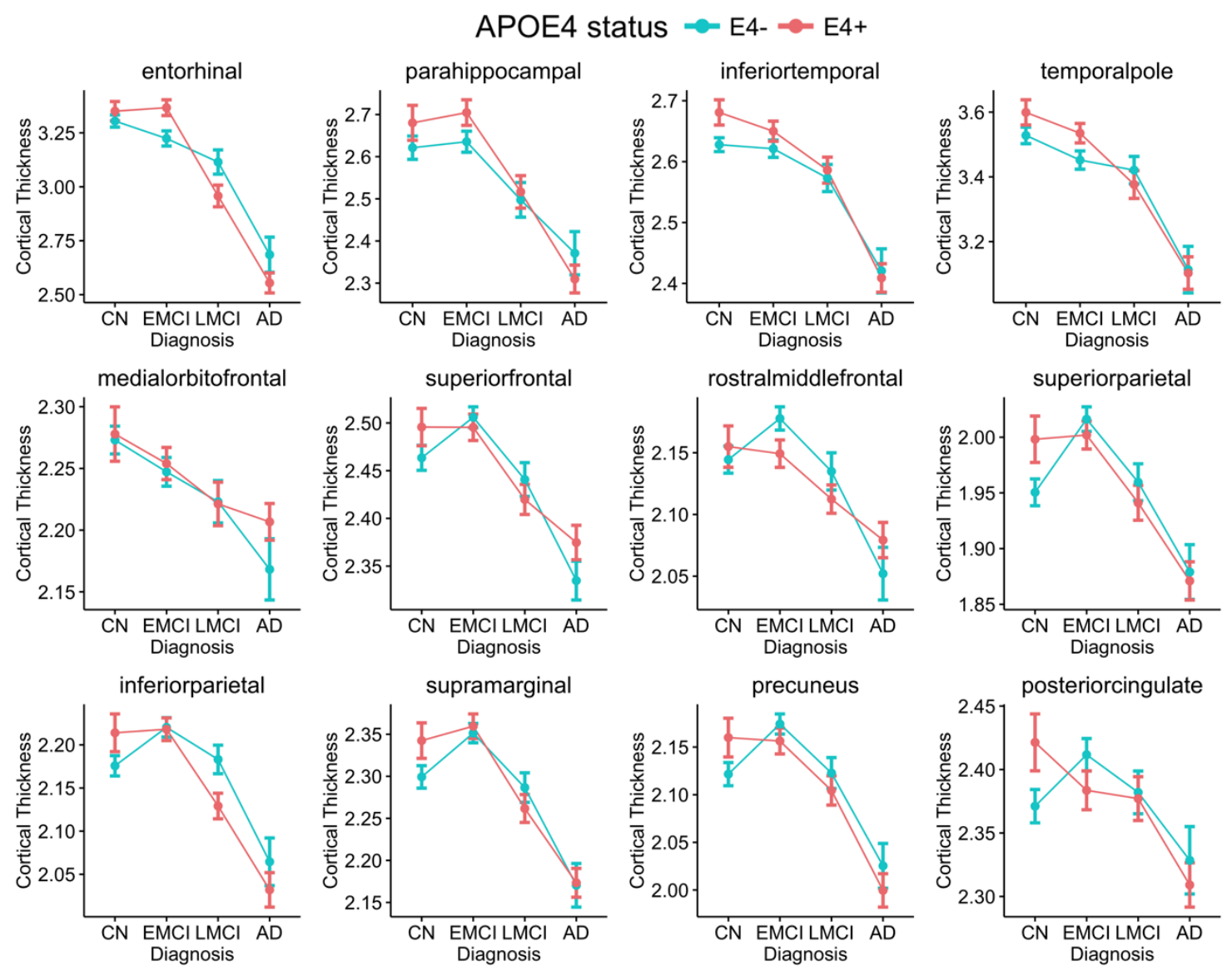

Fig. 10. Barplot of CTh among E4- and E4+ participants in 4 diagnostic groups for 12 brain regions.

\subsubsection{Combined Effect of APOE4 Status and Aß Load Status on CTh among CN and EMCI Groups}

Among $\mathrm{CN}$ and EMCI groups, there were prominent age effects on $\mathrm{CTh}$ in almost every brain region, each with a different combination of $A \beta$ and APOE4 status. However, once such age effects were accounted for, there was no difference in CTh measure in these two groups in any brain region. 
Table 14. Effect of APOE4 status on regional CTh in CN and EMCI, independent of A $\beta$ load (left hemisphere)

\begin{tabular}{|c|c|c|c|c|c|}
\hline & $\begin{array}{c}\mathbf{C N} \\
\text { E4- } 184 \\
\text { E4+67 }\end{array}$ & $\begin{array}{c}\text { EMCI } \\
\text { E4- } 169 \\
\text { E4+ } 128\end{array}$ & $\begin{array}{c}\mathbf{F}^{\mathrm{a}} \\
\text { APOE4 } \\
\text { Status }\end{array}$ & $\begin{array}{c}\mathbf{F}^{\mathrm{a}} \\
\text { Diagnosis }\end{array}$ & $\begin{array}{c}\mathbf{F}^{\mathrm{a}} \\
\text { Diagnosis by } \\
\text { APOE4 }\end{array}$ \\
\hline Entorhinal & $\begin{array}{c}3.30(0.38)^{b} \\
3.35(0.37)\end{array}$ & $\begin{array}{l}3.22(0.46) \\
3.37(0.41)\end{array}$ & $9.68 * * \mathrm{c}$ & 0.26 & 1.92 \\
\hline Parahippocampal & $\begin{array}{l}2.62(0.38) \\
2.68(0.34)\end{array}$ & $\begin{array}{l}2.64(0.32) \\
2.70(0.34)\end{array}$ & $6.02 *$ & 0.71 & 0.06 \\
\hline Inferior temporal & $\begin{array}{l}2.63(0.15) \\
2.68(0.17)\end{array}$ & $\begin{array}{l}2.62(0.19) \\
2.65(0.19)\end{array}$ & $12.56 * * *$ & 0.45 & 0.37 \\
\hline Temporal pole & $\begin{array}{l}3.53(0.35) \\
3.60(0.32)\end{array}$ & $\begin{array}{l}3.45(0.37) \\
3.53(0.34)\end{array}$ & $7.47 * *$ & $3.88 *$ & 0.06 \\
\hline Medial orbitofrontal & $\begin{array}{l}2.27(0.15) \\
2.28(0.18)\end{array}$ & $\begin{array}{l}2.25(0.15) \\
2.25(0.15)\end{array}$ & 1.71 & 1.72 & 0.04 \\
\hline Superior frontal & $\begin{array}{l}2.46(0.18) \\
2.50(0.16)\end{array}$ & $\begin{array}{l}2.51(0.14) \\
2.5(0.16)\end{array}$ & 1.21 & 2.56 & 1.93 \\
\hline Rostral Middle Frontal & $\begin{array}{l}2.14(0.15) \\
2.15(0.14)\end{array}$ & $\begin{array}{l}2.18(0.12) \\
2.15(0.13)\end{array}$ & 0.45 & 1.23 & 2.46 \\
\hline Superior Parietal & $\begin{array}{l}1.95(0.16) \\
2.00(0.17)\end{array}$ & $\begin{array}{l}2.02(0.14) \\
2.00(0.14)\end{array}$ & 1.68 & $6.29 *$ & $4.65^{*}$ \\
\hline Inferior Parietal & $\begin{array}{l}2.18(0.16) \\
2.21(0.18)\end{array}$ & $\begin{array}{l}2.22(0.15) \\
2.22(0.15)\end{array}$ & 2.7 & 3.56 . & 1.81 \\
\hline Supramarginal & $\begin{array}{l}2.30(0.18) \\
2.34(0.17)\end{array}$ & $\begin{array}{l}2.35(0.15) \\
2.36(0.16)\end{array}$ & 3.81 & $5.71 *$ & 1.17 \\
\hline Precuneus & $\begin{array}{l}2.12(0.16) \\
2.16(0.17)\end{array}$ & $\begin{array}{l}2.17(0.14) \\
2.16(0.15)\end{array}$ & 1.6 & $3.89 *$ & 3.58 \\
\hline Posterior Cingulate & $\begin{array}{l}2.37(0.18) \\
2.42(0.18)\end{array}$ & $\begin{array}{l}2.41(0.17) \\
2.38(0.17)\end{array}$ & 0.56 & 0.02 & $5.87 *$ \\
\hline Mean CTh_left & $\begin{array}{l}2.29(0.14) \\
2.32(0.13)\end{array}$ & $\begin{array}{l}2.32(0.11) \\
2.32(0.12)\end{array}$ & 3.55 . & 2.52 & 2.72 . \\
\hline
\end{tabular}

${ }^{\mathrm{a}} \mathrm{F}$ value is adjusted for global A $\beta$ load (2 X 2 ANCOVA test)

${ }^{\mathrm{b}}$ Values are represented as mean(SD), upper is for E4- group and lower is for E4+ group

${ }^{\mathrm{c}} \mathrm{p}<.1 ; * \mathrm{p}<.05 ; * * \mathrm{p}<.01 ; * * * \mathrm{p}<.001$

\subsubsection{Combined Effect of APOE4 Status and A $\beta$ Load Status on Cognitive Variables among CN and EMCI Groups}

From Table 15 and Fig. 11, it can be seen that after accounting for age, there was a significant effect on all assessed cognitive scores: (a) the E4+/Amy+ group showed more impairment than the E4-/Amy- group on the MMSE score $(\mathrm{p}<0.001)$; (b) the E4+/Amy+ 
group was more impaired than the E4-/Amy- $(\mathrm{p}<0.001)$, as well as the E4-/Amy+ groups $(\mathrm{p}=0.005)$ on the RAVLT (immediate) memory test; (c) the E4+/Amy+ group was more impaired that the E4-/Amy- group on the RAVLT percent forgetting $(\mathrm{p}=0.018)$; and $(\mathrm{d})$ the E4+/Amy+ group had more impaired ADAS13 scores as compared to all other combinations of E4+/- and Amy +/- (all p $<0.001)$.

Table 15. Combined effect of APOE4 status and A $\beta$ load status on cognitive scores among $\mathrm{CN}$ and EMCI groups

\begin{tabular}{|c|c|c|c|c|c|c|}
\hline & $\begin{array}{l}\text { E4-Amy- } \\
\text { (241) }\end{array}$ & $\begin{array}{l}\text { E4-Amy+ } \\
\text { (112) }\end{array}$ & $\begin{array}{l}\text { E4+Amy- } \\
\text { (73) }\end{array}$ & $\begin{array}{l}\text { E4+Amy+ } \\
(122)\end{array}$ & F_age & F_E4Amy ${ }^{a}$ \\
\hline MMSE & $28.93(1.35)^{b}$ & $28.54(1.39)$ & $28.73(1.28)$ & $28.16(1.73)$ & $8.48^{* * \mathrm{c}}$ & $\begin{array}{l}7.86^{* * * *} \\
\text { E4+Amy }+<\text { E4- } \\
\text { Amy- }^{d}\end{array}$ \\
\hline $\begin{array}{l}\text { RAVLT } \\
\text { immediate }\end{array}$ & $44.00(10.98)$ & 41.61(11.2) & $43.73(10.8)$ & $38.13(10.3)$ & $36.85^{* * *}$ & $\begin{array}{l}8.23 * * * \\
\text { E4+Amy+<E4- } \\
\text { Amy-; } \\
\text { E4+Amy+<E4- } \\
\text { Amy+ }\end{array}$ \\
\hline $\begin{array}{l}\text { RAVLT } \\
\text { Percent } \\
\text { forgetting }\end{array}$ & $38.79(29.8)$ & $43.67(28.5)$ & $39.8(26.94)$ & $48.37(29.74)$ & $8.5^{* *}$ & $\begin{array}{l}2.86^{*} \\
\text { E4+Amy+ > E4- } \\
\text { Amy- }\end{array}$ \\
\hline ADAS13 & $10.00(4.82)$ & $11.45(5.61)$ & $9.70(5.17)$ & $13.58(5.56)$ & $21.9^{* * *}$ & $\begin{array}{l}13.51 * * * \\
\text { E4+Amy+> All }\end{array}$ \\
\hline
\end{tabular}

\footnotetext{
${ }^{a} \mathrm{~F}$ value is adjusted for age (One-way ANCOVA test)

${ }^{\mathrm{b}}$ Values are represented as mean(SD)

${ }^{c} \mathrm{p}<.1 ;{ }^{*} \mathrm{p}<.05 ; * * \mathrm{p}<.01 ; * * * \mathrm{p}<.001$

${ }^{\mathrm{d}}$ Post Hoc Tukey results with significant difference

${ }^{\mathrm{e}}$ Higher scores indicate worse performance
} 

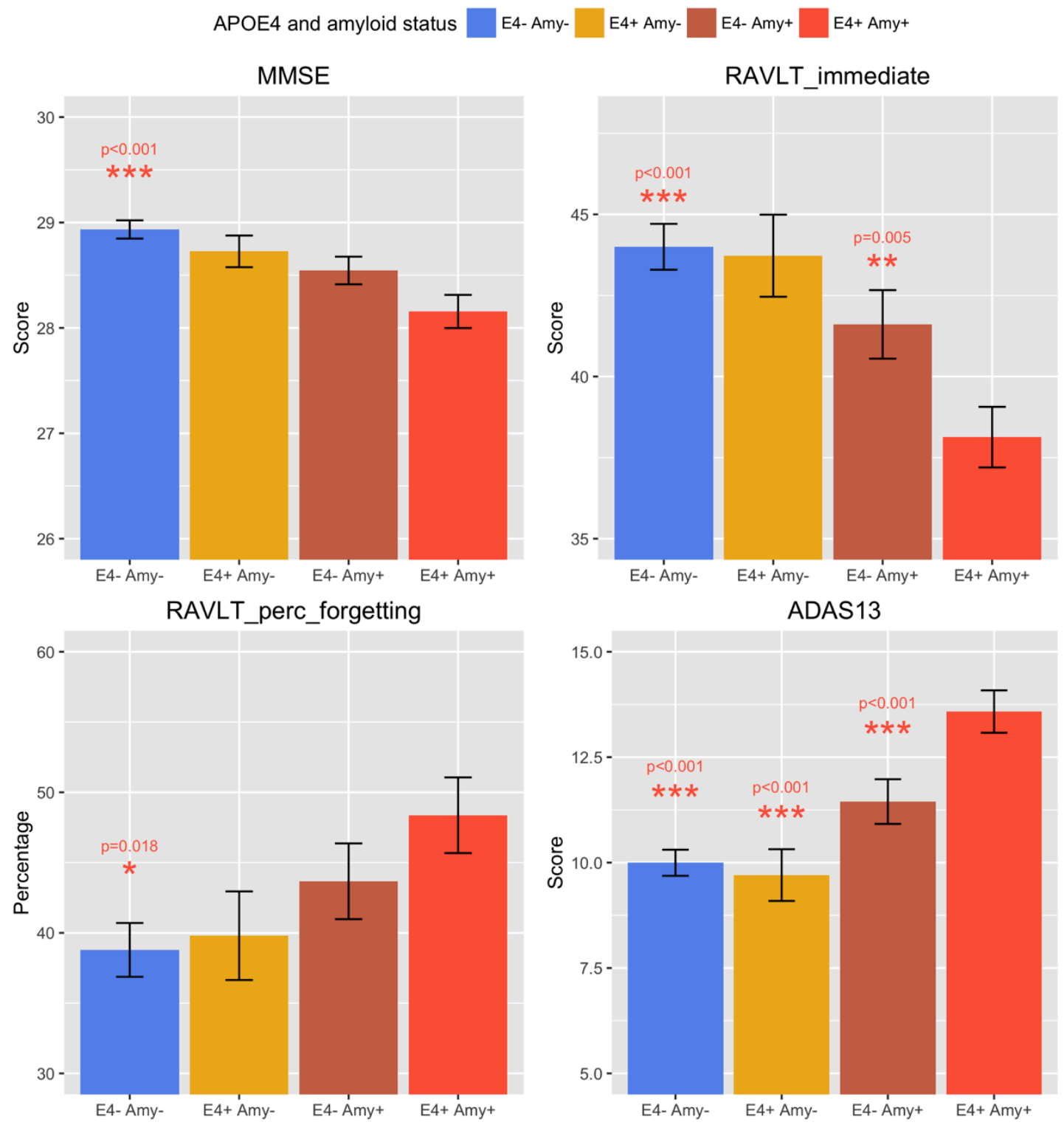

Fig. 11. Bar graph of scores on following cognitive tests: MMSE, RAVLT (immediate), RAVLT (\% forgetting) and ADAS13. Individual bars represent the following groups of participants: E4-/Amy-; E4+/Amy-; E4-/Amy+ and E4+/Amy+. The colored asterisk $\left({ }^{*} \mathrm{p}<.05 ; * * \mathrm{p}<.01 ; * * * \mathrm{p}<.001\right)$ on the E4/Amy- indicates a significant difference in score from the score for the corresponding color bar, i.e., E4+/Amy+ group, same as E4-/Amy+ bars in RAVLT (immediate) and ADAS13, as well as E4+/Amy- in ADAS13. There was no significant difference among E4-/Amy-, E4+/Amy-, E4-/Amy+ group. 


\subsection{Discussion}

This study represents a first attempt to disentangle the complex inter-relationships between $\mathrm{A} \beta$ load, APOE4 genotype, regional $\mathrm{CTh}$ and cognition among well-defined diagnostic groups in ADNI. Previous studies have shown that: (1) higher global A $\beta$ load and $\mathrm{E} 4+$ status are associated with a greater risk of progression from $\mathrm{CN}$ to $\mathrm{MCI}$, and MCI to AD $[84,85]$; (2) higher $\mathrm{A} \beta$ load is associated with reduced $\mathrm{CTh}$, but with subtle impairment of cognition in the $\mathrm{CN}$ and MCI stage [15, 86-94]; (3) E4+ status is associated with an earlier age of onset of $A \beta$ positivity and of $A D$, greater $A \beta$ levels in the brain, reduced hippocampal volumes and $\mathrm{CTh}$ in limbic and neocortical regions, and subtle cognitive deficits in $\mathrm{CN}$ individuals [23, 24, 57, 95-103]; (4) further, irrespective of APOE4 status, reduced hippocampal volumes and CTh in various brain regions, especially those regions characterized as $\mathrm{AD}$ signature regions, are associated with impaired memory and general cognition, as well as a greater risk for progression from $\mathrm{CN}$ to $\mathrm{MCI}$ and MCI to $\mathrm{AD}[104]$. However, there is currently no consensus regarding the extent to which A $\beta$ load status and APOE4 status, independently and in combination, are associated with neurodegenerative changes in AD prone regions and with cognitive impairment.

It is important to note that both trophic and toxic effects of $A \beta$ peptide are known and they may not necessarily be mutually exclusive. The toxic effects may be mediated by different mechanisms, such as oxidative stress, inflammation, mitochondrial diffusion, alterations in membrane permeability, synaptic dysfunction, and excitotoxicity through its interaction with neurotransmitter receptors. These effects contribute significantly to 
the neuronal damage seen in $\mathrm{AD}$, which may be associated with $\mathrm{A} \beta$ itself, including high concentrations in fibrillar or aggregated states, interaction with free metals, interactions with previously injured or aged brain tissue and with decreased antioxidative mechanisms [105-108].

We devised our analytic strategy to investigate, among 906 participants in the ADNI1/ADNI-Go and ADNI-2 cohorts, the unique and independent contributions of those elements, which are considered as upstream factors (i.e., A $\beta$ load status and APOE4 status), on downstream factors (regional $\mathrm{CTh}$ and cognitive measures tapping memory and general cognition). We also investigated the individual and additive effects of E4+ and Amy+ status on CTh and cognitive performance, in the earliest stages of neurodegeneration, using different combinations of APOE4 status and A $\beta$ load status (E4-/Amy-, E4+/Amy-, E4-/Amy+ and E4+/Amy+) among CN and EMCI participants.

Our results confirmed previous reports showing that E4+ status is associated with increased $\mathrm{A} \beta$ load among all stages of $\mathrm{AD}$ (Table 11). Amy+ status, among all participants, was found to be associated with reduced CTh in many AD vulnerable regions, independent of the effects of E4+ status (Table 12) [89, 90, 109, 110], but E4+ status was associated with reduced CTh in restricted brain regions, and only among LMCI and AD participants (Table 13). Unexpectedly, E4+ status was associated with increased $\mathrm{CTh}$ in some of the most vulnerable brain regions to AD pathology (i.e., the ERC, PHG and ITG) in the preclinical and very early stages of AD (i.e., among CN and EMCI participants) (Table 14). 
Even though we found there was no observed cortical thinning in association with Amy+ status, and there was cortical thickening in association with E4+ status, among $\mathrm{CN}$ and EMCI participants, there was impairment on all cognitive tests among these participants, in association with either Amy+ and E4+ status, independently and to the greatest extent with combined Amy+ and E4+ status (Table 15). Global cognitive scores (ADAS13), among $\mathrm{CN}$ and EMCI participants, were significantly impaired among E4+/Amy + groups as compared to all other groups, including those who were E4+/Amy-, suggesting that the presence of Amy+ status is independently associated with greater impairment of global cognitive scores. Further, memory impairment, on the RAVLT immediate test, was greater among those who were E4-/Amy+, as compared to those who were E4+/Amy+, suggesting that the presence of E4+ status is independently associated with greater immediate memory impairment. These results demonstrate that in the earliest stages of $\mathrm{AD}$, in the absence of cortical thinning, there is impairment in cognitive performance attributable to combined E4+ and Amy+ status, as well as independently to E4+ status and to Amy+ status. Given that there is a known relationship between CTh and cognitive scores $[104,111,112]$, the finding among $\mathrm{CN}$ and EMCI participants, who are E4+, of an association with increased $\mathrm{CTh}$ in the most vulnerable regions to AD pathology, suggests that the mechanism underlying neurodegeneration associated with E4+ status is distinct from that of Amy+ status.

Biological processes, such as metabolic activation, increased blood flow and inflammation $[113,114]$, may result in increased volume of the cortical ribbon in brain regions, in which the neurodegenerative process begins, with subsequent cortical thinning 
as neurodegeneration becomes more advanced [115-117]. It is apparent that APOE4 is a contributing factor to neurodegeneration, and is strongly linked to AD pathology, alone and particularly in combination with the $\mathrm{A} \beta$ peptide. APOE4 may increase A $\beta$ deposition in plaques and impair its clearance, and also may act independently through pathways that may not involve A $\beta$ [118]. The pathophysiological effects of APOE4 may be mediated at a molecular level in the process of redistribution of lipids in normal lipid homeostasis, repairing injured neurons, maintaining synapto-dendritic connections, and scavenging toxins. These pathophysiological effects result in adverse outcomes in various neurological conditions and in "normal" aging. E4+ status is associated with adverse outcomes, acceleration of progression, worsening overall prognosis in response to head injury, oxidative stress, ischemia, and inflammation, as well as lowering of the age of onset of neurodegenerative disease [115].

A weakness of this study is that it is cross-sectional and so any inferences about progression from the $\mathrm{CN}$ to $\mathrm{AD}$ stage must be considered tentative. A major strength of the current investigation is that it utilizes a well-characterized ADNI cohort, including large numbers of subjects who are cognitively normal or in the earliest stages of disease, to power the analyses. These large numbers of subjects that are available in the ADNI cohort provide the statistical power required to tease apart independent effects of upstream biological processes, such as $\mathrm{A} \beta$ load and APOE4 status as they relate to downstream effects on CTh and cognition. Future analyses should attempt to confirm these findings using longitudinal analyses of the data, and to further evaluate the effects of regional, rather than global deposition of $\mathrm{A} \beta$, on $\mathrm{CTh}$ and cognition. 
In conclusion, our findings suggest that both $\mathrm{A} \beta$ load and APOE4 status are highly associated with progressive neurodegeneration, as measured by cortical thinning, especially in the LMCI and $\mathrm{AD}$ stages, and especially in brain regions which are vulnerable to AD pathology. Even though there is no significant cortical thinning noted in $\mathrm{CN}$ and EMCI stages, associated with E4+ or Amy+ status, there is measurable cognitive impairment present. The association of E4+ status with cortical thickening, rather than thinning, suggests the possibility of a very different pathophysiological role for E4+ from that of $\mathrm{A} \beta$ deposition in the progression of $\mathrm{AD}$. 


\section{CHAPTER V}

\section{DIVERGING ASSOCIATION PATTERNS OF REGIONAL CORTICAL ATROPHY WITH GLOBAL AND WITH REGIONAL AMYLOID DEPOSITION}

\subsection{Goal}

Previous studies that have examined the effects among $\mathrm{A} \beta$ deposition and gray matter atrophy were either based on the mean global neocortical $\mathrm{A} \beta$ ratio or on the voxel-tovoxel level [119-127]. The mean global neocortical A $\beta$ ratio method classified individuals into $A \beta$ normal level ( $A \beta$ negative) and $A \beta$ abnormal level (A $\beta$ positive), and compared the regional brain atrophy of those two groups within or across different disease stages. Nevertheless, this global way might overlook the direct assessment of brain amyloidosis and neurofibrillary pathology in specific regions. Although the voxelbased approach is simple and a direct way to assess the relationship of the brain atrophy and $A \beta$ load in each voxel, regional information is often ignored in many studies.

In the present investigation, we aimed to assess and compare the association patterns of regional and global $\mathrm{A} \beta$ load with brain atrophy in $\mathrm{AD}$-vulnerable and AD-unrelated regions, across difference stages of the disease (CN, EMCI, LMCI and AD), considering the mediated performance of APOE4. We further explored the relationship of global A $\beta$ load and regional $A \beta$ deposition in regions that showed different association patterns. Specifically, we tested the following hypotheses: (i) the association patterns with brain atrophy is more robust for regional $A \beta$ load than for global $A \beta$ load; (ii) the relationship 
between $A \beta$ load and brain atrophy is region-specific and disease-stage-specific; and (iii) regional $\mathrm{A} \beta$ accumulation is not always linearly related to the global $\mathrm{A} \beta$ load.

\subsection{Materials and Methods}

\subsubsection{Study Participants}

Data and image processing method used in the preparation of this article is same as in the chapter II.

\subsubsection{Regions of Interest}

In this study, we tested 12 relatively vulnerable AD-related neurodegeneration regions in both hemispheres: entorhinal, temporal pole, parahippocampal, fusiform, inferior temporal, precuneus, posterior cingulate, isthmus cingulate, inferior parietal, supramarginal, rostral middle frontal, and insula. For contrasting purposes, we also

considered 4 relatively resistant regions: precentral, postcentral, pericalcarine, and cuneus.

\subsubsection{Statistical analysis}

To compare subjects' characteristics among the diagnostic stages: CN, EMCI, LMCI and $\mathrm{AD}$, we performed analysis of variance (ANOVA) test on age, year of education, MMSE score, and CDRSB score, as well as the Chi-square test on gender and APOE4 gene. 
Considering the mediated performance of APOE4 on the aggregated forms of A $\beta$ [24] and on the cortical atrophy [128], we performed Pearson partial correlation (PPC) method to assess the relationship between regional/global $A \beta$ load and regional cortical atrophy within each diagnostic group, controlling for the number of APOE4 alleles, age, and the total intracranial volume (ICV). Multiple-comparison corrections were then considered by controlling false discovery rate (FDR) per diagnostic group. As a secondary analysis, we used ANOVA method to examine whether the regional/global $A \beta$ load or the regional volume was different across the spectrum of disease. Following a statistically significant F-test, the post hoc Tukey HSD procedure was then employed for subgroup comparisons.

To investigate whether the relationship between regional and global $A \beta$ accumulation is linear or assumed a more non-linear function, we introduced the LOESS regression model to fit the curve of the $A \beta$ load in a specific region and in the whole cortical among all participants. In this study, the quadratic model and the smooth parameter were considered.

All statistical analyses were performed using R software (R 3.3.0), a well known software environment for statistical computing and graphics [83]. The default significant level was determined as 0.05 . 


\subsubsection{Pearson Partial Correlation (PPC)}

Pearson Partial Correlation is a measure of the linear relationship between two continuous variables controlling for the effect of on ore more other variables. The partial correlation coefficient represents the strength and direction of such relation.

Take the right precuneus cortical region in EMCI group for example. Let $x_{i}, y_{i}, z_{i}^{1}, z_{i}^{2}, z_{i}^{3}$, be the volume, the SUVR of such region, the number of APOE4 alleles, age and the ICV of patient $i,(i=1,2,3, \ldots, 296)$, respectively. First of all, the linear regression model for $\mathrm{x}$ or $\mathrm{y}$ and three covariance $\mathrm{z}^{1}, \mathrm{z}^{2}, \mathrm{z}^{3}$ is built (5.1), from which the fitted values $\hat{x}_{i}$ and $\hat{y}_{i}$, as well as the residuals $\tilde{x}_{i}, \tilde{y}_{i}$ are calculated.

$$
\begin{aligned}
& \hat{x}=\alpha+\beta_{1} z^{1}+\beta_{2} z^{2}+\beta_{3} z^{3} \\
& \hat{y}=\omega+\gamma_{1} z^{1}+\gamma_{2} z^{2}+\gamma_{3} z^{3}
\end{aligned}
$$

The PPC between $\mathrm{x}$ and $\mathrm{y}$ controlling for $\mathrm{z}^{1}, \mathrm{z}^{2}$, and $\mathrm{z}^{3}\left(\mathrm{r}_{\mathrm{xy} \mid \mathrm{z}}\right)$ is the Pearson correlation (5.2) between the residuals of the $\mathrm{x}$ and $\mathrm{y}$ after regression on $\mathrm{z}^{1}, \mathrm{z}^{2}, \mathrm{z}^{3}$.

$$
r_{x y \mathrm{z} z}=\frac{\sum_{i=1}^{296}\left(\tilde{x}_{i}-\mu_{\tilde{x}}\right)\left(\tilde{y}_{i}-\mu_{\tilde{y}}\right)}{\sqrt{\sum_{i=1}^{296}\left(\tilde{x}_{i}-\mu_{\tilde{x}}\right)^{2} \sum_{i=1}^{296}\left(\tilde{y}_{i}-\mu_{\tilde{y}}\right)^{2}}}
$$

where, $\mu_{\tilde{x}}=\frac{1}{296} \sum_{i=1}^{296} \tilde{x}_{i}$ and similarly to $\mu_{\tilde{y}}$. 


\subsubsection{Locally Weighted Scatterplot Smoothing (LOESS) Regression}

The LOESS is the local polynomial regression and nonparametric method. It fits simple polynomial models to localized subsets of the data point by point. The number of points considered in each subset is defined by the smoothing parameter $\alpha$, i.e., the proportion of data that is considered to be neighboring that point. Suppose the data size is n, at each point, at say $x$, the polynomial $Y=X \beta$ is fitted using weighted lease squares (5.3), i.e., giving more weight to points near $x$, and less weight to points far away.

$$
\hat{\beta}=\left(X^{T} W X\right)^{-1} X^{T} W Y
$$

where, tricubic weighting (5.5) is used.

$$
\begin{aligned}
& W=\left[\begin{array}{cccc}
w_{1} & 0 & 0 & 0 \\
0 & w_{2} & 0 & 0 \\
0 & 0 & \ddots & 0 \\
0 & 0 & 0 & w_{k}
\end{array}\right] \\
& w_{i}=\left[1-\left[\frac{\left\|x_{i}-x\right\|^{2}}{\operatorname{Max}_{j=1}^{k}\left\|x_{j}-x\right\|^{2}}\right]^{3}\right]^{3}
\end{aligned}
$$

where $k=n \alpha$.

The LOESS Curve is then created based on regression function values that have been computed for each of the $\mathrm{n}$ points. In short, the LOESS combines $\mathrm{n}$ linear least square regression models in a k-nearest-neighbor-based-meta mode. 


\subsection{Results}

As indicated in Table 4 in Chapter II, significant demographic differences was found only for age. The presence of APOE4 was expectedly highest in AD patients, and intermediated in EMCI and LMCI groups. What follows are the main findings of this study detailed in three sections which express (1) the associations of cortical atrophy with global $A \beta$ load, (2) the associations of cortical atrophy with regional $A \beta$ load, and (3) the relationship between regional and global $\mathrm{A} \beta$ load.

\subsubsection{Associations of cortical atrophy with global A $\beta$ load}

As expected, in $\mathrm{AD}$-vulnerable regions, there was considerable negative correlation between the whole brain $A \beta$ load and the cortical volume, i.e., higher $A \beta$ deposition is associated with greater cortical atrophy, which was detected in AD and its prodromal stages of EMCI and LMCI, whereas no significant relationship was found in the CN stage (Figure 12A and Table 16). Specifically, inferior parietal $(\mathrm{p}=0.004)$, isthmus cingulate $(\mathrm{p}=0.021)$ in the left hemisphere and supramarginal $(\mathrm{p}=0.008)$, parahippocampal $(\mathrm{p}=0.025)$, isthmus cingulate $(\mathrm{p}=0.025)$, insula $(0.026)$, precuneus (0.035) in right hemisphere started to show minor but statistically significant negative patterns in the EMCI stage, with partial correlation coefficients $r$ in range -0.123 to -0.17 . The inferior parietal (left: $\mathrm{p}=0.038$, right: $\mathrm{p}=0.009$ ), precuneus (left: $\mathrm{p}=0.028$, right: $\mathrm{p}$ $=0.010$ ), fusiform (left: $\mathrm{p}=0.033$, right: $\mathrm{p}=0.003$ ), and insula (left: $\mathrm{p}=0.019$, right: $\mathrm{p}=$ 0.020) persisted with or were even strengthened in the LMCI stage. 
In addition, similar patterns extended to the following regions in LMCI stage: entorhinal (left: $\mathrm{p}=0.024$, right: $\mathrm{p}=0.019$ ), inferior temporal and posterior cingulate (significant in left: $\mathrm{p}=0.001$ and 0.002 , mild in right: $\mathrm{p}=0.099$ and 0.0504 , respectively), left supramarginal $(\mathrm{p}=0.003)$ and left temporal pole $(\mathrm{p}=0.044)$. The range of the partial correlation coefficient in the LMCI stage was from -0.146 to -0.24 . In AD stage, such pattern remained in bilateral inferior temporal (left: $p=0.037$, right: $p=0.01$ ), inferior parietal (left: $\mathrm{p}<0.001$, right: $\mathrm{p}=0.003$ ), right isthmus cingulate $(\mathrm{p}=0.022)$, and left precuneus (0.042), extending to rostral middle frontal (left: $p=0.01$, right: $p=0.012$ ).

No significant pattern was discovered in any AD-resistant regions except for left cuneus, in which negative relationship was shown in AD stage $(p=0.031)$. All associations were mild and generally not significant after correction for multiple comparisons (corrected for PPC 32 tests), and the associations that remained significant was in left inferior temporal, left posterior cingulate, left supramarginal and right fusiform in LMCI stage and in bilateral inferior parietal cortex in AD stage (Table 17). 
A.

Global SUVR $\sim$ Regional Volume Regional SUVR $\sim$ Regional Volume Controlling for Age, APOE4, ICV Controlling for Age, APOE4, ICV

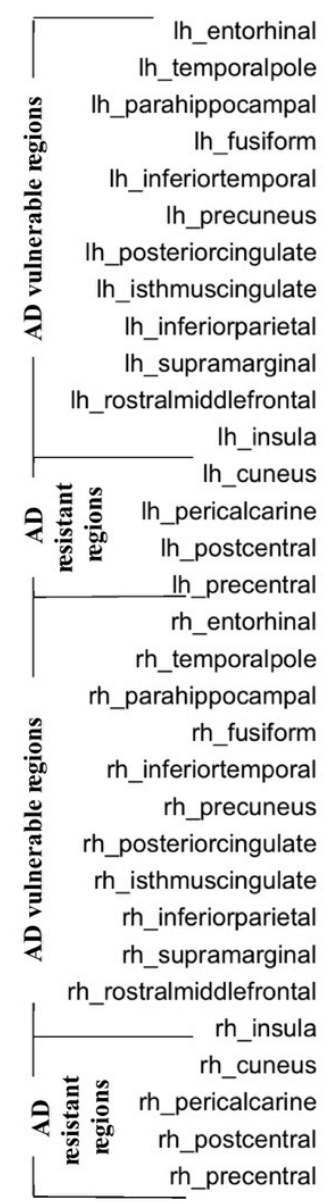

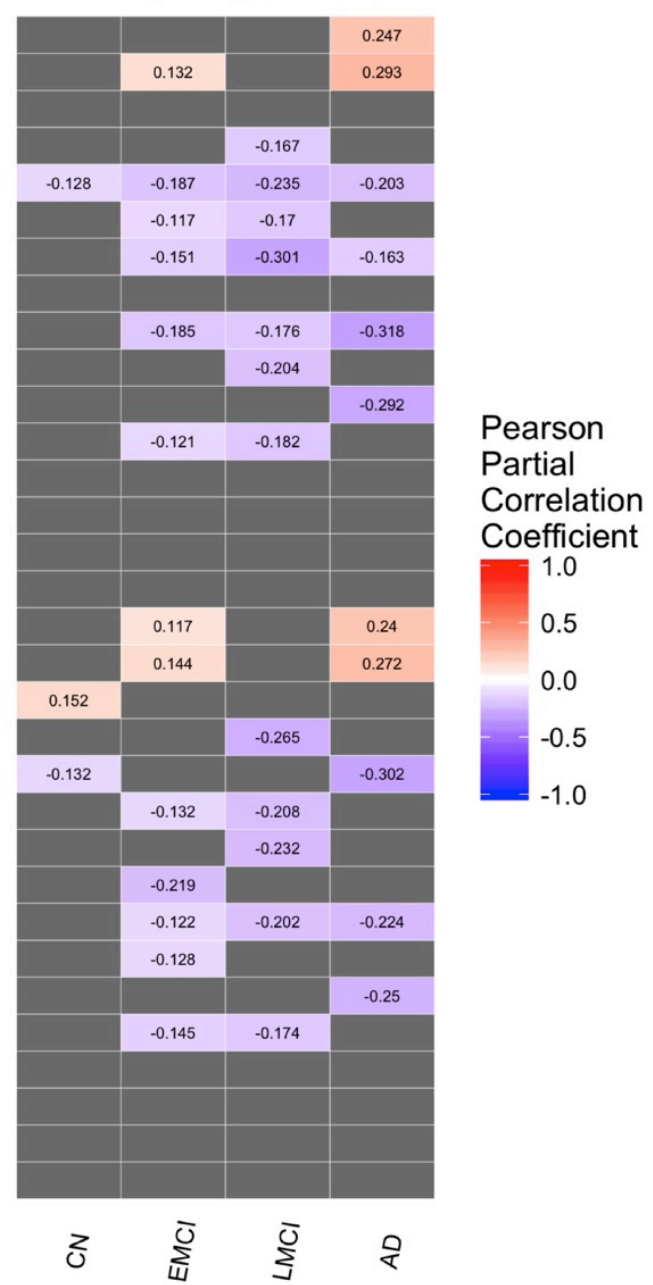

Fig. 12. Association patterns of cortical atrophy with global $A \beta$ load (A), and regional $A \beta$ load (B), displayed as heatmap with partial correlation coefficients displayed at $\mathrm{p}$ (uncorrected) $<0.05$. 
Table 16. Associations of cortical atrophy and global $A \beta$ load

\begin{tabular}{|c|c|c|c|c|}
\hline Region & $\mathrm{CN}$ & EMCI & LMCI & AD \\
\hline lh_entorhinal ${ }^{\mathrm{a}}$ & $-0.073(0.251)^{b}$ & $-0.057(0.329)$ & $-0.164(0.024)$ & $0.071(0.38)$ \\
\hline lh_temporalpole & $-0.035(0.588)$ & $0.015(0.802)$ & $-0.146(0.044)$ & $0.087(0.28)$ \\
\hline lh parahippocampal & $-0.081(0.205)$ & $-0.105(0.073)$ & $-0.087(0.232)$ & $-0.141(0.08)$ \\
\hline lh fusiform & $0.004(0.945)$ & $-0.056(0.339)$ & $-0.155(0.033)$ & $-0.155(0.053)$ \\
\hline lh_inferiortemporal & $-0.109(0.088)$ & $-0.075(0.201)$ & $-0.24(0.001)$ & $-0.167(0.037)$ \\
\hline lh_precuneus & $0.038(0.554)$ & $-0.092(0.114)$ & $-0.16(0.028)$ & $-0.163(0.042)$ \\
\hline lh posteriorcingulate & $-0.031(0.626)$ & $-0.083(0.156)$ & $-0.229(0.002)$ & $-0.113(0.16)$ \\
\hline lh_isthmuscingulate & $0.028(0.659)$ & $-0.135(0.02)$ & $-0.064(0.381)$ & $-0.102(0.205)$ \\
\hline lh_inferiorparietal & $0.054(0.401)$ & $-0.17(0.004)$ & $-0.151(0.038)$ & $-0.319(0)$ \\
\hline lh_supramarginal & $0.106(0.096)$ & $-0.065(0.27)$ & $-0.211(0.003)$ & $-0.028(0.727)$ \\
\hline lh rostralmiddlefrontal & $-0.005(0.94)$ & $-0.086(0.142)$ & $-0.043(0.557)$ & $-0.205(0.01)$ \\
\hline lh_insula & $0.013(0.835)$ & $-0.072(0.218)$ & $-0.17(0.019)$ & $-0.035(0.662)$ \\
\hline lh_cuneus & $0.077(0.23)$ & $0.052(0.372)$ & $0.049(0.5)$ & $-0.173(0.03)$ \\
\hline 1h_pericalcarine & $0.033(0.603)$ & $0.08(0.172)$ & $0.03(0.678)$ & $-0.129(0.108)$ \\
\hline lh postcentral & $0.03(0.636)$ & $0.021(0.716)$ & $-0.043(0.553)$ & $-0.114(0.158)$ \\
\hline lh_precentral & $0.074(0.249)$ & $0.063(0.284)$ & $0.017(0.815)$ & $-0.009(0.915)$ \\
\hline rh_entorhinal & $-0.091(0.156)$ & $-0.085(0.146)$ & $-0.171(0.019)$ & $0.047(0.563)$ \\
\hline rh_temporalpole & $0.005(0.94)$ & $-0.083(0.156)$ & $-0.108(0.138)$ & $0.135(0.092)$ \\
\hline rh parahippocampal & $0.105(0.101)$ & $-0.131(0.025)$ & $-0.132(0.069)$ & $-0.126(0.118)$ \\
\hline rh_fusiform & $-0.008(0.902)$ & $-0.102(0.082)$ & $-0.212(0.003)$ & $-0.103(0.202)$ \\
\hline rh inferiortemporal & $-0.069(0.278)$ & $-0.08(0.17)$ & $-0.12(0.099)$ & $-0.206(0.01)$ \\
\hline rh_precuneus & $-0.037(0.565)$ & $-0.123(0.035)$ & $-0.188(0.01)$ & $-0.127(0.113)$ \\
\hline rh_posteriorcingulate & $0.011(0.859)$ & $0.012(0.835)$ & $-0.142(0.05)$ & $0.071(0.376)$ \\
\hline rh_isthmuscingulate & $0.023(0.719)$ & $-0.13(0.026)$ & $0.01(0.892)$ & $-0.184(0.022)$ \\
\hline rh_inferiorparietal & $-0.017(0.787)$ & $-0.098(0.092)$ & $-0.19(0.009)$ & $-0.237(0.003)$ \\
\hline rh_supramarginal & $0.112(0.079)$ & $-0.154(0.008)$ & $-0.06(0.414)$ & $-0.023(0.78)$ \\
\hline rh_rostralmiddlefrontal & $-0.057(0.369)$ & $-0.067(0.254)$ & $0.036(0.62)$ & $-0.201(0.012)$ \\
\hline rh_insula & $0.036(0.572)$ & $-0.13(0.026)$ & $-0.168(0.02)$ & $0.061(0.453)$ \\
\hline rh_cuneus & $0.108(0.089)$ & $-0.03(0.607)$ & $0.065(0.371)$ & $-0.077(0.34)$ \\
\hline rh pericalcarine & $0.113(0.077)$ & $0.065(0.266)$ & $-0.064(0.38)$ & $-0.136(0.09)$ \\
\hline rh_postcentral & $0.096(0.133)$ & $-0.001(0.985)$ & $-0.005(0.948)$ & $-0.048(0.551)$ \\
\hline rh_precentral & $0.091(0.154)$ & $0.032(0.59)$ & $0(0.996)$ & $0.062(0.444)$ \\
\hline
\end{tabular}

${ }^{\mathrm{a}} \mathrm{lh}=$ left hemisphere; $\mathrm{rh}=$ right hemisphere

${ }^{b}$ Values are represented as Pearson Partial Correlation coefficient (uncorrected $p$ value), with age, number of APOE4 and ICV as covariance. Tests significant at $p<0.05$ are printed in bold. 
Table 17. Associations of cortical atrophy and global $A \beta$ load (corrected $p$ value)

\begin{tabular}{|c|c|c|c|c|}
\hline Region & $\mathbf{C N}$ & EMCI & LMCI & AD \\
\hline lh_entorhinal ${ }^{\mathrm{a}}$ & $-0.073(0.617)^{b}$ & $-0.057(0.434)$ & $-0.164(0.077)$ & $0.071(0.506)$ \\
\hline lh_temporalpole & $-0.035(0.878)$ & $0.015(0.855)$ & $-0.146(0.1)$ & $0.087(0.426)$ \\
\hline lh_parahippocampal & $-0.081(0.617)$ & $-0.105(0.291)$ & $-0.087(0.392)$ & $-0.141(0.227)$ \\
\hline lh_fusiform & $0.004(0.945)$ & $-0.056(0.434)$ & $-0.155(0.088)$ & $-0.155(0.171)$ \\
\hline lh_inferiortemporal & $-0.109(0.537)$ & $-0.075(0.357)$ & $-0.24(0.024)$ & $-0.167(0.148)$ \\
\hline lh_precuneus & $0.038(0.878)$ & $-0.092(0.324)$ & $-0.16(0.081)$ & $-0.163(0.148)$ \\
\hline 1h_posteriorcingulate & $-0.031(0.878)$ & $-0.083(0.324)$ & $-0.229(0.024)$ & $-0.113(0.284)$ \\
\hline lh_isthmuscingulate & $0.028(0.878)$ & $-0.135(0.14)$ & $-0.064(0.554)$ & $-0.102(0.328)$ \\
\hline lh_inferiorparietal & $0.054(0.802)$ & $-0.17(0.112)$ & $-0.151(0.094)$ & $-0.319(0)$ \\
\hline lh_supramarginal & $0.106(0.537)$ & $-0.065(0.393)$ & $-0.211(0.027)$ & $-0.028(0.775)$ \\
\hline lh_rostralmiddlefrontal & $-0.005(0.945)$ & $-0.086(0.324)$ & $-0.043(0.686)$ & $-0.205(0.077)$ \\
\hline lh_insula & $0.013(0.945)$ & $-0.072(0.367)$ & $-0.17(0.072)$ & $-0.035(0.73)$ \\
\hline lh_cuneus & $0.077(0.617)$ & $0.052(0.458)$ & $0.049(0.667)$ & $-0.173(0.139)$ \\
\hline lh_pericalcarine & $0.033(0.878)$ & $0.08(0.324)$ & $0.03(0.775)$ & $-0.129(0.236)$ \\
\hline lh_postcentral & $0.03(0.878)$ & $0.021(0.79)$ & $-0.043(0.686)$ & $-0.114(0.284)$ \\
\hline lh_precentral & $0.074(0.617)$ & $0.063(0.396)$ & $0.017(0.899)$ & $-0.009(0.915)$ \\
\hline rh_entorhinal & $-0.091(0.554)$ & $-0.085(0.324)$ & $-0.171(0.072)$ & $0.047(0.643)$ \\
\hline rh_temporalpole & $0.005(0.945)$ & $-0.083(0.324)$ & $-0.108(0.244)$ & $0.135(0.227)$ \\
\hline rh_parahippocampal & $0.105(0.537)$ & $-0.131(0.14)$ & $-0.132(0.138)$ & $-0.126(0.236)$ \\
\hline rh_fusiform & $-0.008(0.945)$ & $-0.102(0.292)$ & $-0.212(0.027)$ & $-0.103(0.328)$ \\
\hline rh_inferiortemporal & $-0.069(0.636)$ & $-0.08(0.324)$ & $-0.12(0.186)$ & $-0.206(0.077)$ \\
\hline rh_precuneus & $-0.037(0.878)$ & $-0.123(0.161)$ & $-0.188(0.051)$ & $-0.127(0.236)$ \\
\hline rh_posteriorcingulate & $0.011(0.945)$ & $0.012(0.862)$ & $-0.142(0.108)$ & $0.071(0.506)$ \\
\hline rh_isthmuscingulate & $0.023(0.921)$ & $-0.13(0.14)$ & $0.01(0.952)$ & $-0.184(0.116)$ \\
\hline rh_inferiorparietal & $-0.017(0.945)$ & $-0.098(0.296)$ & $-0.19(0.051)$ & $-0.237(0.046)$ \\
\hline rh_supramarginal & $0.112(0.537)$ & $-0.154(0.131)$ & $-0.06(0.576)$ & $-0.023(0.805)$ \\
\hline rh_rostralmiddlefrontal & $-0.057(0.788)$ & $-0.067(0.393)$ & $0.036(0.735)$ & $-0.201(0.077)$ \\
\hline rh_insula & $0.036(0.878)$ & $-0.13(0.14)$ & $-0.168(0.072)$ & $0.061(0.557)$ \\
\hline rh_cuneus & $0.108(0.537)$ & $-0.03(0.693)$ & $0.065(0.554)$ & $-0.077(0.495)$ \\
\hline rh_pericalcarine & $0.113(0.537)$ & $0.065(0.393)$ & $-0.064(0.554)$ & $-0.136(0.227)$ \\
\hline rh_postcentral & $0.096(0.554)$ & $-0.001(0.985)$ & $-0.005(0.979)$ & $-0.048(0.643)$ \\
\hline rh_precentral & $0.091(0.554)$ & $0.032(0.693)$ & $0(0.996)$ & $0.062(0.557)$ \\
\hline
\end{tabular}

${ }^{\mathrm{a}} \mathrm{lh}=$ left hemisphere; $\mathrm{rh}=$ right hemisphere

${ }^{\mathrm{b}}$ Values are represented as Pearson Partial Correlation coefficient (corrected $\mathrm{p}$ value per diagnostic group using FDR), with age, number of APOE4 and ICV as covariance. Tests significant at $\mathrm{p}<0.05$ are printed in bold.

\subsubsection{Associations of cortical atrophy with regional $A \beta$ load}

In general, compared with the global $A \beta, 17$ out of 32 regions of interest (ROI) showed exactly the same patterns in the regional $A \beta$ level (4.3.2.1); 6 out of 32 ROIs showed similar patterns as in global $\mathrm{A} \beta$, while extended to other diagnostic stages (4.3.2.2); 4 of 
the 32 ROIs did not show the significant negative associations either in the regional level or in global level (4.3.2.3); and 5 ROIs showed unexpectedly reversed patterns, which was generally negative in global level, but positive in regional level (4.3.2.4).

\subsubsection{Regions showing same patterns with global $A \beta$}

Compared with the global $A \beta, 17$ out of 32 regions of interest (ROI) showed exactly the same but generally stronger patterns in the regional $A \beta$ level. Among these 17 ROIs, negative correlation was detected in 9 ROIs: bilateral fusiform (left: $\mathrm{p}=0.021$, right: $\mathrm{p}<$ 0.001 in LMCI), left inferior parietal $(p=0.002,0.015,<0.001$ in EMCI, LMCI and AD respectively), right precuneus ( $\mathrm{p}=0.023$ and 0.004 in EMCI and LMCI, respectively), bilateral rostral middle frontal (left: $\mathrm{p}<0.001$, right: $\mathrm{p}=0.002$ in AD) and right insula ( $\mathrm{p}$ $=0.013,0.016$ in EMCI and LMCI, respectively), bilateral supramarginal (left: $p=0.005$ in LMCI, right: $\mathrm{p}=0.028$ in EMCI). On the other hand, there was no significant relationship detected in any of the prodromal stages in the following 8 ROIs: left parahippocampal, bilateral precentral, bilateral postcentral, bilateral pericalcarine, as well as right cuneus (Fig. 12B and Table 18).

Taking insight into the right precuneus ROI, for example, similar association patterns can also be found from the linear regression models of volume and regional (Fig. 13A), as well as global (Fig. 13B), A $\beta$ load. Both regional and global $\mathrm{A} \beta$ deposition showed significant negative associations with volume in EMCI $(p=0.012$ and 0.007, respectively) and LMCI stages ( $\mathrm{p}=0.033$ and 0.048 , respectively), and also in AD stage for global $A \beta$ load $(p=0.027)$. 
Table 18. Associations of cortical atrophy and regional A $\beta$ load

\begin{tabular}{|c|c|c|c|c|}
\hline Region & $\mathbf{C N}$ & EMCI & LMCI & AD \\
\hline lh_entorhinal ${ }^{\mathrm{a}}$ & $0.078(0.22)^{b}$ & $0.087(0.138)$ & $0.033(0.651)$ & $0.247(0.002)$ \\
\hline lh_temporalpole & $0.092(0.152)$ & $0.132(0.024)$ & $0.01(0.894)$ & $0.293(0)$ \\
\hline lh_parahippocampal & $-0.004(0.954)$ & $-0.093(0.113)$ & $-0.027(0.71)$ & $-0.136(0.091)$ \\
\hline lh_fusiform & $-0.01(0.874)$ & $-0.102(0.081)$ & $-0.167(0.021)$ & $-0.136(0.089)$ \\
\hline lh_inferiortemporal & $-0.128(0.044)$ & $-0.187(0.001)$ & $-0.235(0.001)$ & $-0.203(0.011)$ \\
\hline lh_precuneus & $0.03(0.639)$ & $-0.117(0.046)$ & $-0.17(0.019)$ & $-0.143(0.076)$ \\
\hline lh_posteriorcingulate & $-0.092(0.148)$ & $-0.151(0.01)$ & $-0.301(0)$ & $-0.163(0.042)$ \\
\hline lh_isthmuscingulate & $-0.006(0.931)$ & $-0.111(0.057)$ & $-0.102(0.163)$ & $0.013(0.872)$ \\
\hline lh_inferiorparietal & $0.04(0.526)$ & $-0.185(0.002)$ & $-0.176(0.015)$ & $-0.318(0)$ \\
\hline lh_supramarginal & $0.068(0.284)$ & $-0.062(0.292)$ & $-0.204(0.005)$ & $0.02(0.808)$ \\
\hline lh_rostralmiddlefrontal & $-0.032(0.62)$ & $-0.1(0.087)$ & $-0.07(0.336)$ & $-0.292(0)$ \\
\hline lh_insula & $0.02(0.761)$ & $-0.121(0.038)$ & $-0.182(0.012)$ & $-0.032(0.696)$ \\
\hline lh_cuneus & $-0.006(0.929)$ & $0.012(0.845)$ & $0.018(0.804)$ & $-0.106(0.189)$ \\
\hline lh_pericalcarine & $0.001(0.99)$ & $0.024(0.687)$ & $-0.056(0.442)$ & $-0.116(0.148)$ \\
\hline lh_postcentral & $0.01(0.869)$ & $0.072(0.219)$ & $-0.045(0.534)$ & $-0.116(0.148)$ \\
\hline lh_precentral & $0.084(0.187)$ & $0.043(0.461)$ & $0.027(0.71)$ & $-0.032(0.692)$ \\
\hline rh_entorhinal & $-0.102(0.111)$ & $0.117(0.045)$ & $0.056(0.442)$ & $0.24(0.003)$ \\
\hline rh_temporalpole & $0.036(0.572)$ & $0.144(0.013)$ & $0.11(0.13)$ & $0.272(0.001)$ \\
\hline rh_parahippocampal & $0.152(0.017)$ & $-0.043(0.462)$ & $-0.105(0.15)$ & $0.044(0.589)$ \\
\hline rh_fusiform & $-0.06(0.347)$ & $-0.108(0.064)$ & $-0.265(0)$ & $-0.127(0.114)$ \\
\hline rh_inferiortemporal & $-0.132(0.039)$ & $-0.09(0.124)$ & $-0.129(0.076)$ & $-0.302(0)$ \\
\hline rh_precuneus & $-0.06(0.351)$ & $-0.132(0.023)$ & $-0.208(0.004)$ & $-0.118(0.143)$ \\
\hline rh_posteriorcingulate & $-0.062(0.333)$ & $-0.065(0.264)$ & $-0.232(0.001)$ & $0.035(0.668)$ \\
\hline rh_isthmuscingulate & $-0.038(0.554)$ & $-0.219(0)$ & $-0.024(0.745)$ & $-0.063(0.435)$ \\
\hline rh_inferiorparietal & $-0.045(0.479)$ & $-0.122(0.037)$ & $-0.202(0.005)$ & $-0.224(0.005)$ \\
\hline rh_supramarginal & $0.092(0.148)$ & $-0.128(0.028)$ & $-0.069(0.344)$ & $-0.026(0.747)$ \\
\hline rh_rostralmiddlefrontal & $-0.083(0.195)$ & $-0.08(0.17)$ & $-0.005(0.942)$ & $-0.25(0.002)$ \\
\hline rh_insula & $0.017(0.785)$ & $-0.145(0.013)$ & $-0.174(0.016)$ & $0.063(0.436)$ \\
\hline rh_cuneus & $0.054(0.396)$ & $-0.024(0.68)$ & $0.02(0.788)$ & $-0.011(0.889)$ \\
\hline rh_pericalcarine & $0.06(0.35)$ & $0.062(0.293)$ & $-0.114(0.117)$ & $-0.055(0.492)$ \\
\hline rh_postcentral & $0.057(0.373)$ & $-0.007(0.9)$ & $0.003(0.965)$ & $-0.026(0.745)$ \\
\hline rh_precentral & $0.112(0.079)$ & $0.001(0.992)$ & $-0.006(0.937)$ & $0.063(0.434)$ \\
\hline
\end{tabular}

The performance of the $\mathrm{A} \beta$ load in right precuneus (Fig. 13D) was consistent with that of the global $A \beta$ burden (Fig. 13E): significant increased $A \beta$ accumulation compared with previous diagnostic stages, i.e. EMCI vs. CN, LMCI vs. CN and EMCI, as well as AD vs. $\mathrm{CN}$, EMCI and LMCI, which further confirmed the aforementioned same association 
patterns. In terms of the cortical atrophy (Fig. 13C), LMCI and AD patients showed significant decreased cortical volume compared to EMCI and to CN, EMCI and LMCI, respectively (all $\mathrm{p}<0.001$ ). Such cortical thinning was not detected when comparing EMCI to CN. However, EMCI patients showed larger cortical volume $(\mathrm{p}<0.001)$, which was still significant after correcting for age $(p=0.004)$.

\subsubsection{Regions with stronger relations}

Six out of 32 ROIs showed similar patterns as in global $A \beta$, which extended to other diagnostic stages. Compared with the global $\mathrm{A} \beta(\mathrm{r}=-0.24,-0.167$ in LMCI and AD, respectively), left inferior temporal showed similar negative patterns in LMCI $(\mathrm{r}=$ $0.239, \mathrm{p}=0.001)$ and stronger in $\mathrm{AD}(\mathrm{r}=-0.203, \mathrm{p}=0.011)$, and extended such trend to $\mathrm{CN}(\mathrm{p}=0.045)$ and EMCI $(\mathrm{p}=0.001)$ as well. Similar to left posterior cingulate (stronger pattern in LMCI ( $r=-0.301$ vs. -0.229 in global), extended to EMCI and AD with $p$ $<0.001,=0.010,0.042$ in LMCI, EMCI and AD, respectively), left insular (mild stronger pattern in LMCI ( $\mathrm{r}=-0.182$ vs. -0.17$)$, and extended to EMCI with $\mathrm{p}=0.012$ and 0.028 , respectively), left precuneus (similar pattern in $\mathrm{LMCI}$, mild trend in $\mathrm{AD}$, and extended to EMCI with $\mathrm{p}=0.019,0.076,0.046$, respectively), right inferior temporal (stronger pattern in $\mathrm{AD}(\mathrm{r}=-0.302$ vs. -0.206$)$, mild trend in LMCI, and extended to $\mathrm{CN}$ with $\mathrm{p}$ $<0.001,=0.076,0.039$, respectively), and right inferior parietal (similar patterns in LMCI and $\mathrm{AD}$, extended to EMCI with $\mathrm{p}=0.005,0.005,0.037$, respectively). 


\section{rh_precuneus}

A

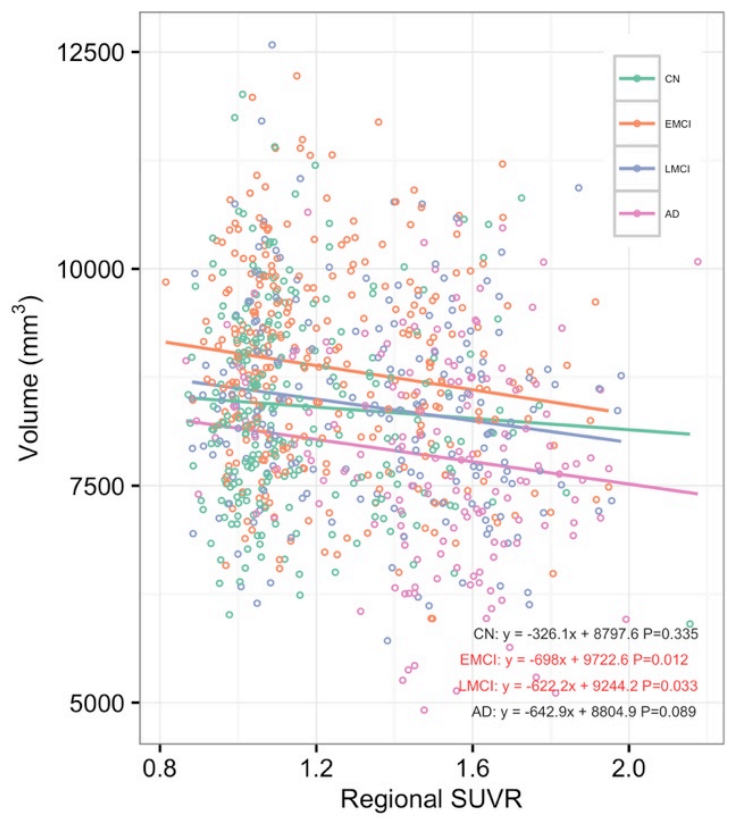

C

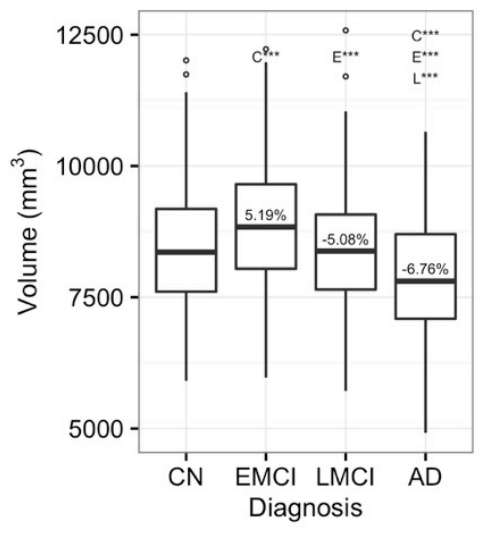

B

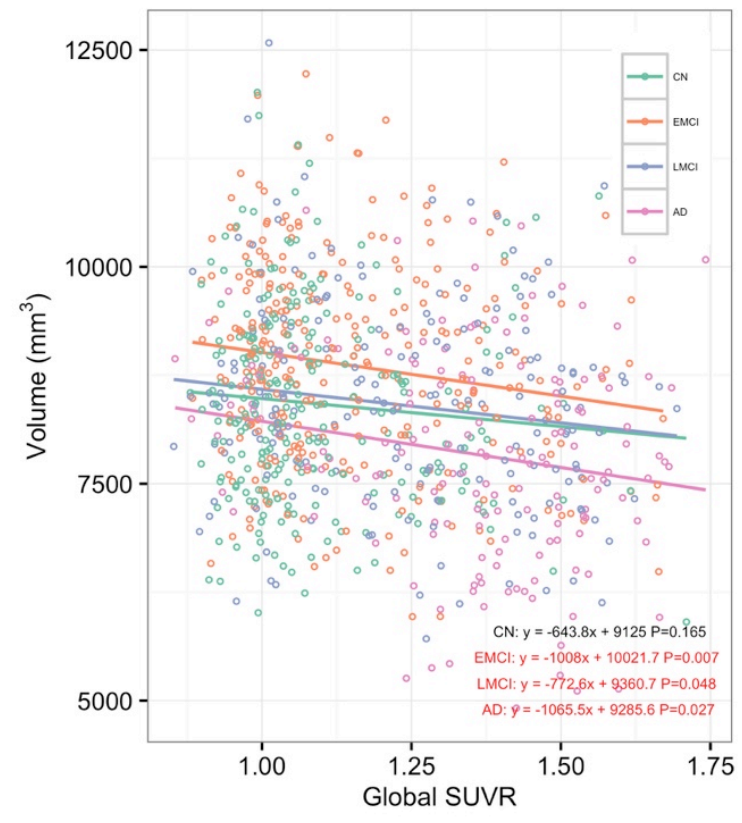

D

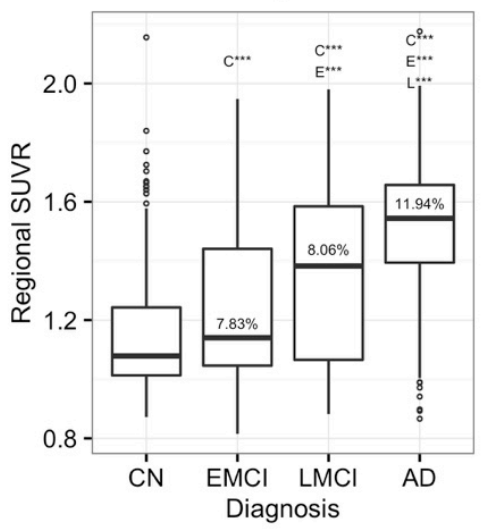

E

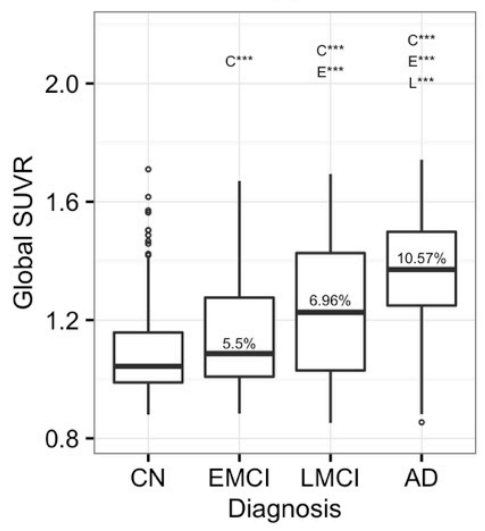

Fig. 13. Linear regression models and performance of volume, regional $A \beta$ load and global $A \beta$ load in right precuneus. (A) Scatterplot of the regional A $\beta$ load and volume. The fitted lines are from linear regression models for each diagnosis stage, with formulas listed at the right bottom as well as the $\mathrm{p}$ values for regional $A \beta$ standardized uptake value ratio (SUVR) in the linear regression models. (B) Scatterplot and the linear regression models of global $A \beta$ load and regional volume. (C) Boxplot of volumes across all diagnostic stages: CN, EMCI, LMCI and AD. C, E, and L above the boxes represented a significant difference in the volume from CN, EMCI, LMCI group, respectively along with the significant level: ${ }^{*} \mathrm{p}<.05 ;{ }^{*} \mathrm{p}<.01$; $* * * p<.001$. Values in the box indicated the mean volume change rate from the previous stage $(5.19 \%$ in the EMCI box denotes that on average, the volume in the EMCI group is $5.19 \%$ larger than that in CN group.) (D) Boxplot of regional $A \beta$ load across 4 diagnostic stages, represented by the regional A $\beta$ SUVR. (E) Boxplot of global A $\beta$ load across 4 diagnostic stages, represented by the whole cortical A $\beta$ SUVR. 


\subsubsection{Regions with weaker relationships}

Three of the 32 ROIs did not show the significant negative associations as detected in global level: left isthmus cingulate in EMCI $(p=0.057)$, left cuneus in AD $(p=0.189)$, and right isthmus cingulate in $\mathrm{AD}(\mathrm{p}=0.435)$. On the other hand, in right posterior cingulate ROI, the negative relationship reached significant level in LMCI $(p=0.001)$ compared to the mild trend in global level $(\mathrm{p}=0.0504)$.

A.

Global SUVR Regional Volume Controlling for Age, APOE4, ICV Controlling for Age, APOE4, ICV

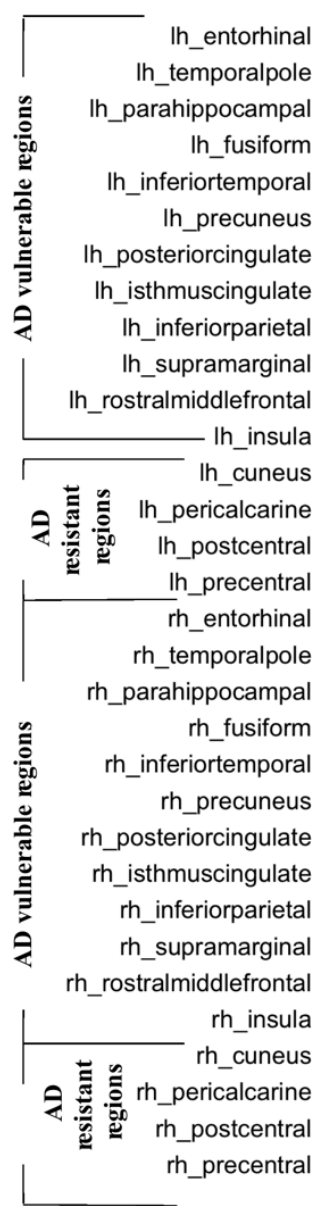

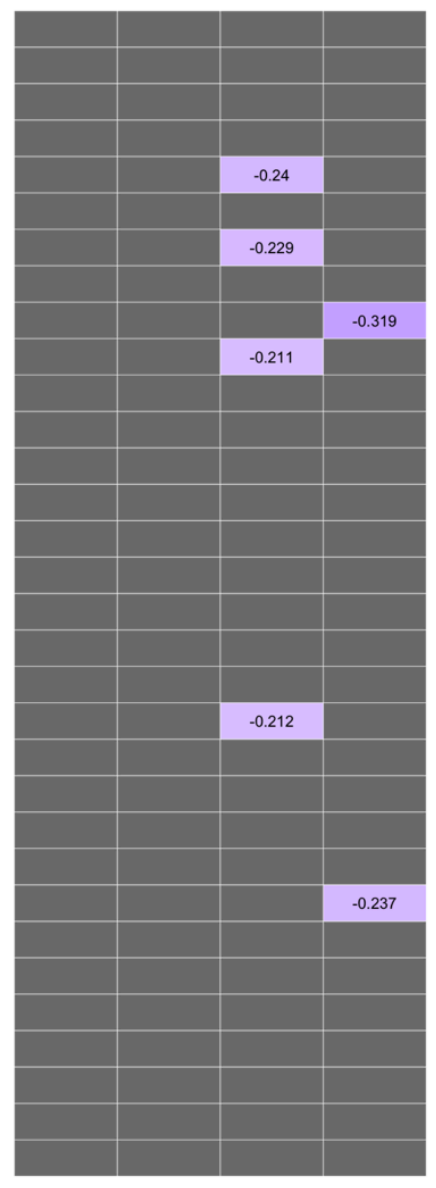

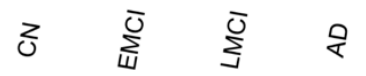

B.

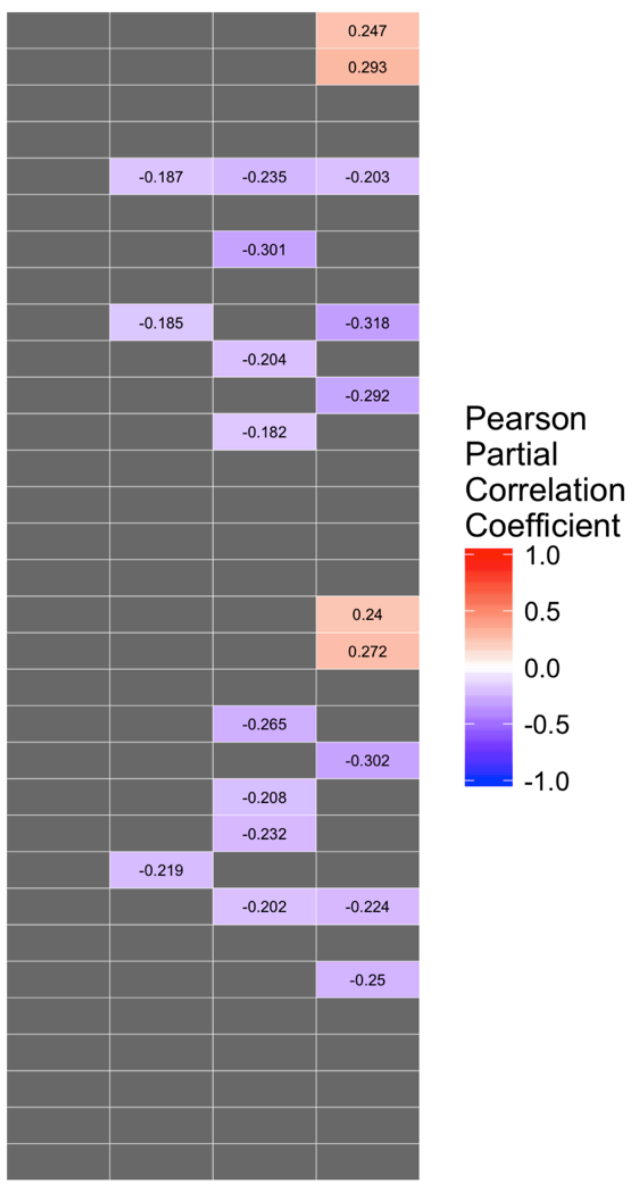

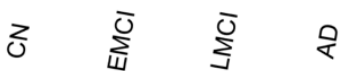

Fig. 14. Association patterns of cortical atrophy with global amyloid load (A), and regional A $\beta$ load (B), displayed as heatmap with partial correlation coefficients displayed at $\mathrm{p}$ (corrected) $<0.05$. 


\subsubsection{Regions with reversed patterns}

There were 5 specific ROIs showing unexpectedly reversed patterns, which was generally negative in global level, but positive in regional level. Specifically, right parahippocampal showed positive correlation in $\mathrm{CN}$ group $(\mathrm{p}=0.017)$, i.e., greater $\mathrm{A} \beta$ load was related to larger cortical volume; bilateral temporal pole (left: $p=0.024$, $<0.001$, right: $\mathrm{p}=0.013,0.001)$ and right entorhinal $(\mathrm{p}=0.045,0.003)$ in EMCI and AD stages, and left entorhinal in $\mathrm{AD}(\mathrm{p}=0.002)$. Half of the significant associations remained significant after multiple-comparison correction (corrected for 32 PPC tests; Fig. 14B).

For right parahippocampal ROI, similar positive correlation in $\mathrm{CN}$ group between volume and regional $A \beta$ deposition was found through the linear regression model (Fig. 15A), along with the negative relationships in EMCI and LMCI stages between volume and global $A \beta$ load (Fig. 15B). The performance of the volume was similar to the right precuneus ROI: LMCI and AD patients showed significant decreased cortical volume compared to EMCI and to CN, EMCI and LMCI, respectively ( $p<0.001$ for all), while EMCI patients had larger cortical volume than $\mathrm{CN}(\mathrm{p}=0.03)$ instead of cortical thinning (Fig. 15C), but such pattern was not significant anymore when adjusting for age $(\mathrm{p}=$ 0.68). On the other hand, although the regional $A \beta$ was significantly accumulated in $A D$ stage compared with the other 3 stages, the LMC only showed significant higher A $\beta$ load than $\mathrm{CN}$, while no significant difference was found between $\mathrm{CN}$ and EMCI (Fig. 15D). 
Table 19. Associations of cortical atrophy and global $A \beta$ load (corrected $p$ value)

\begin{tabular}{|c|c|c|c|c|}
\hline Region & $\mathbf{C N}$ & EMCI & LMCI & AD \\
\hline lh_entorhinal ${ }^{\mathrm{a}}$ & $0.078(0.641)^{b}$ & $0.087(0.22)$ & $0.033(0.905)$ & $0.247(0.009)$ \\
\hline lh_temporalpole & $0.092(0.607)$ & $0.132(0.097)$ & $0.01(0.965)$ & $0.293(0.002)$ \\
\hline lh_parahippocampal & $-0.004(0.985)$ & $-0.093(0.201)$ & $-0.027(0.909)$ & $-0.136(0.208)$ \\
\hline lh_fusiform & $-0.01(0.985)$ & $-0.102(0.162)$ & $-0.167(0.056)$ & $-0.136(0.208)$ \\
\hline lh_inferiortemporal & $-0.128(0.475)$ & $-0.187(0.016)$ & $-0.235(0.01)$ & $-0.203(0.035)$ \\
\hline lh_precuneus & $0.03(0.852)$ & $-0.117(0.113)$ & $-0.17(0.056)$ & $-0.143(0.201)$ \\
\hline lh_posteriorcingulate & $-0.092(0.607)$ & $-0.151(0.071)$ & $-0.301(0)$ & $-0.163(0.122)$ \\
\hline lh_isthmuscingulate & $-0.006(0.985)$ & $-0.111(0.131)$ & $-0.102(0.307)$ & $0.013(0.889)$ \\
\hline lh_inferiorparietal & $0.04(0.832)$ & $-0.185(0.016)$ & $-0.176(0.052)$ & $-0.318(0.002)$ \\
\hline lh_supramarginal & $0.068(0.702)$ & $-0.062(0.375)$ & $-0.204(0.024)$ & $0.02(0.862)$ \\
\hline lh_rostralmiddlefrontal & $-0.032(0.852)$ & $-0.1(0.164)$ & $-0.07(0.579)$ & $-0.292(0.002)$ \\
\hline lh_insula & $0.02(0.966)$ & $-0.121(0.112)$ & $-0.182(0.047)$ & $-0.032(0.824)$ \\
\hline lh_cuneus & $-0.006(0.985)$ & $0.012(0.901)$ & $0.018(0.918)$ & $-0.106(0.319)$ \\
\hline lh_pericalcarine & $0.001(0.99)$ & $0.024(0.758)$ & $-0.056(0.674)$ & $-0.116(0.263)$ \\
\hline lh_postcentral & $0.01(0.985)$ & $0.072(0.319)$ & $-0.045(0.776)$ & $-0.116(0.263)$ \\
\hline lh_precentral & $0.084(0.624)$ & $0.043(0.548)$ & $0.027(0.909)$ & $-0.032(0.824)$ \\
\hline rh_entorhinal & $-0.102(0.607)$ & $0.117(0.113)$ & $0.056(0.674)$ & $0.24(0.01)$ \\
\hline rh_temporalpole & $0.036(0.832)$ & $0.144(0.071)$ & $0.11(0.277)$ & $0.272(0.004)$ \\
\hline rh_parahippocampal & $0.152(0.475)$ & $-0.043(0.548)$ & $-0.105(0.3)$ & $0.044(0.785)$ \\
\hline rh_fusiform & $-0.06(0.702)$ & $-0.108(0.137)$ & $-0.265(0.003)$ & $-0.127(0.243)$ \\
\hline rh_inferiortemporal & $-0.132(0.475)$ & $-0.09(0.209)$ & $-0.129(0.188)$ & $-0.302(0.002)$ \\
\hline rh_precuneus & $-0.06(0.702)$ & $-0.132(0.097)$ & $-0.208(0.024)$ & $-0.118(0.263)$ \\
\hline rh_posteriorcingulate & $-0.062(0.702)$ & $-0.065(0.368)$ & $-0.232(0.01)$ & $0.035(0.824)$ \\
\hline rh_isthmuscingulate & $-0.038(0.832)$ & $-0.219(0.006)$ & $-0.024(0.916)$ & $-0.063(0.635)$ \\
\hline rh_inferiorparietal & $-0.045(0.806)$ & $-0.122(0.112)$ & $-0.202(0.024)$ & $-0.224(0.018)$ \\
\hline rh_supramarginal & $0.092(0.607)$ & $-0.128(0.1)$ & $-0.069(0.579)$ & $-0.026(0.824)$ \\
\hline rh_rostralmiddlefrontal & $-0.083(0.624)$ & $-0.08(0.26)$ & $-0.005(0.965)$ & $-0.25(0.009)$ \\
\hline rh_insula & $0.017(0.966)$ & $-0.145(0.071)$ & $-0.174(0.052)$ & $0.063(0.635)$ \\
\hline rh_cuneus & $0.054(0.705)$ & $-0.024(0.758)$ & $0.02(0.918)$ & $-0.011(0.889)$ \\
\hline rh_pericalcarine & $0.06(0.702)$ & $0.062(0.375)$ & $-0.114(0.268)$ & $-0.055(0.685)$ \\
\hline rh_postcentral & $0.057(0.702)$ & $-0.007(0.929)$ & $0.003(0.965)$ & $-0.026(0.824)$ \\
\hline rh_precentral & $0.112(0.607)$ & $0.001(0.992)$ & $-0.006(0.965)$ & $0.063(0.635)$ \\
\hline
\end{tabular}

${ }^{\mathrm{a}} \mathrm{lh}=$ left hemisphere; $\mathrm{rh}=$ right hemisphere

${ }^{\mathrm{b}}$ Values are represented as Pearson Partial Correlation coefficient (uncorrected $\mathrm{p}$ value), with age, number of APOE4 and ICV as covariance. Tests significant at $p<0.05$ are printed in bold. Tests significant at $\mathrm{p}<0.05$ and with positive partial correlation coefficient are printed in bold and italic.

As for the right entorhinal ROI, according to the linear regression model without adjusting for age, APOE4 and ICV, significant negative association between regional A $\beta$ load and volume was detected in $\mathrm{CN}$ group $(\mathrm{p}=0.006)$, while positive association was found in AD group $(\mathrm{p}=0.012)$. Unlike the PPC test, the positive correlation in EMCI group was mild but not significant $(\mathrm{p}=0.196)$ (Fig. 16A). In terms of the global A $\beta$ load, 
significant negative association was found in CN, EMCI and LMCI stages $(p=0.026$, 0.044, 0.041, respectively) (Fig. 16B). In addition, the LMCI and AD showed dynamically smaller volume than the previous stages (CN, EMCI for LMCI; CN, EMCI, LMCI for AD, respectively, all $\mathrm{p}<0.001$ ), however, such atrophy trend was very slight and not significant in EMCI compared with CN (Fig. 16C). Moreover, only AD group showed significant higher $A \beta$ deposition than its previous stages: $\mathrm{CN}$, EMCI, and LMCI $(\mathrm{p}=0.002,0.024,0.049$, respectively)

\subsubsection{Relationship between regional and global A $\beta$ load}

Based on aforementioned different association patterns between the regional and global $\mathrm{A} \beta$ level, we further investigated the LOESS curve of right entorhinal, right temporal pole, right parahippocampal ROIs, which showed opposite patterns. We also evaluated the fitted LOESS curve of left inferior temporal, right precuneus and left inferior parietal ROIs that presented consistent or even more robust negative patterns. The regional $\mathrm{A} \beta$ load in all the three ROIs with reverse patterns showed non-linearly interaction with global $A \beta$ retention: the regional $A \beta$ SUVR of right entorhinal experienced an increase when the global A $\beta$ SUVR was less than 1.12, while such increasing rate was slowed down after the global A $\beta$ SUVR reached 1.12. A similar but mild pattern was found in the left temporal pole and right parahippocampal. For those ROIs with consistent association patterns, the regional A $\beta$ SUVR seemed linearly related to the global A $\beta$ SUVR, since the LOESS curve was almost straight (Fig. 17). 


\section{rh_parahippocampal}

A

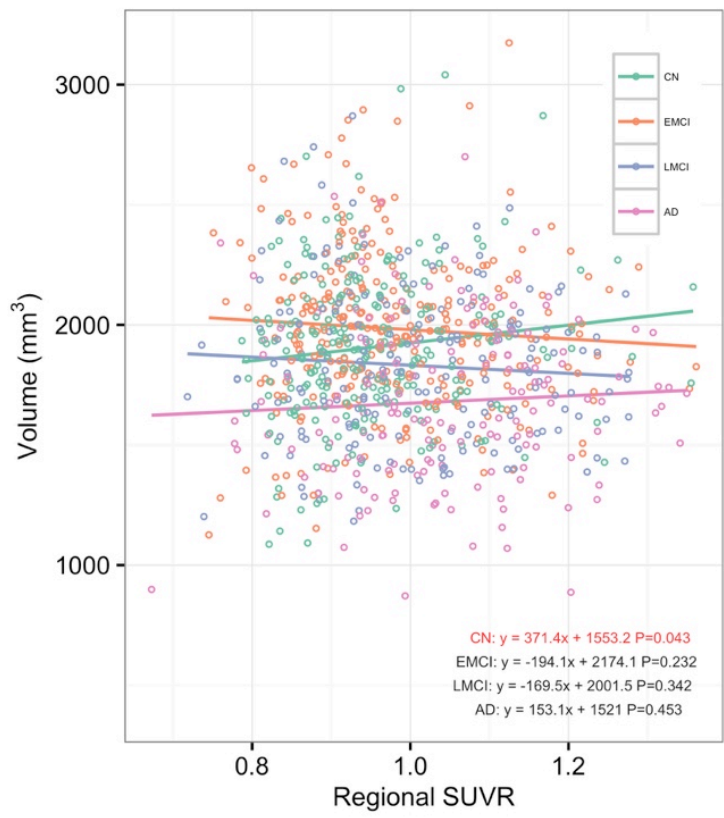

C

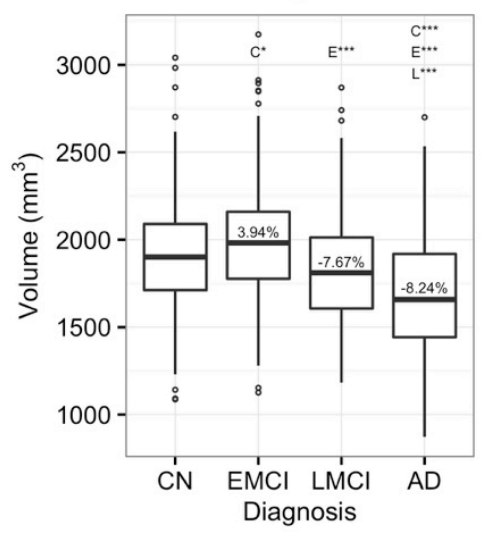

B

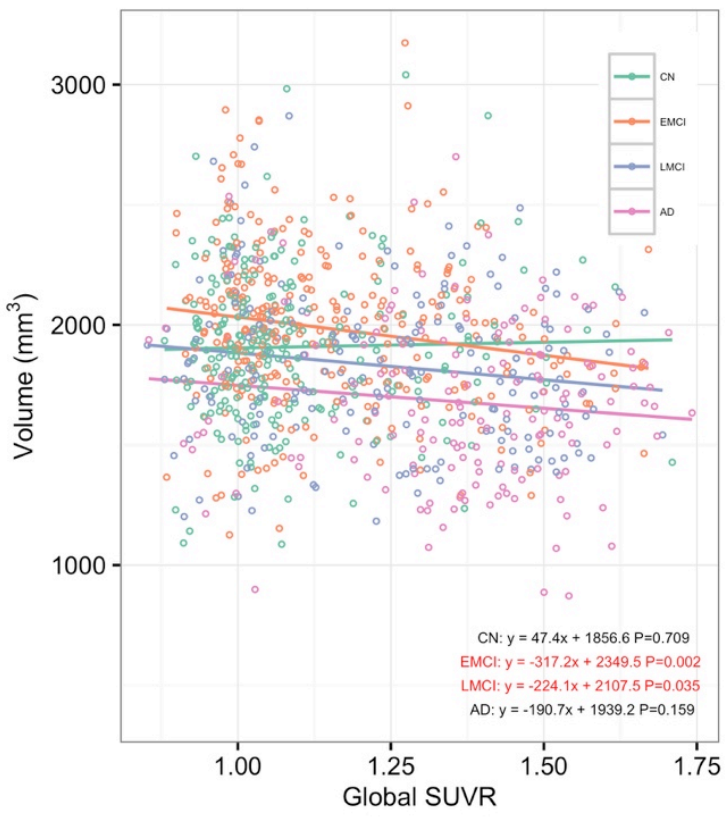

D

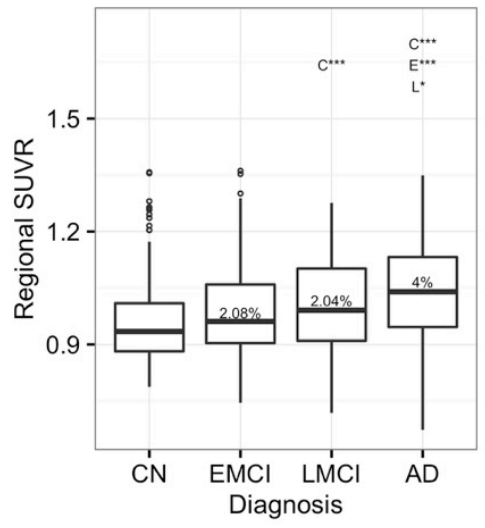

$\mathrm{E}$

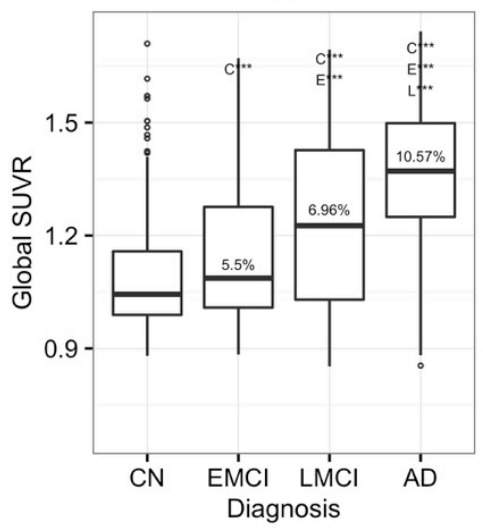

Fig. 15. Linear regression models and performance of volume, regional $A \beta$ load and global $A \beta$ load in right parahippocampal. (A) Scatterplot of the regional $A \beta$ load and volume. The fitted lines are from linear regression models for each diagnosis stage, with formulas listed at the right bottom as well as the $p$ values for regional $A \beta$ standardized uptake value ration (SUVR) in the linear regression models. (B) Scatterplot and the linear regression models of global $\mathrm{A} \beta$ load and regional volume. (C) Boxplot of volumes across all diagnostic stages: $\mathrm{CN}$, EMCI, LMCI and $\mathrm{AD}$. $\mathrm{C}, \mathrm{E}$, and $\mathrm{L}$ above the boxes represented a significant difference in the volume from CN, EMCI, LMCI group, respectively along with the significant level: ${ }^{*} \mathrm{p}$ $<.05 ; * * \mathrm{p}<.01 ; * * * \mathrm{p}<.001$. Values in the box indicated the mean volume change rate from the previous stage. (D) Boxplot of regional A $\beta$ load across 4 diagnostic stages, represented by the regional A $\beta$ SUVR. (E) Boxplot of global A $\beta$ load across 4 diagnostic stages, represented by the whole cortical A $\beta$ SUVR. 


\section{rh_entorhinal}

A

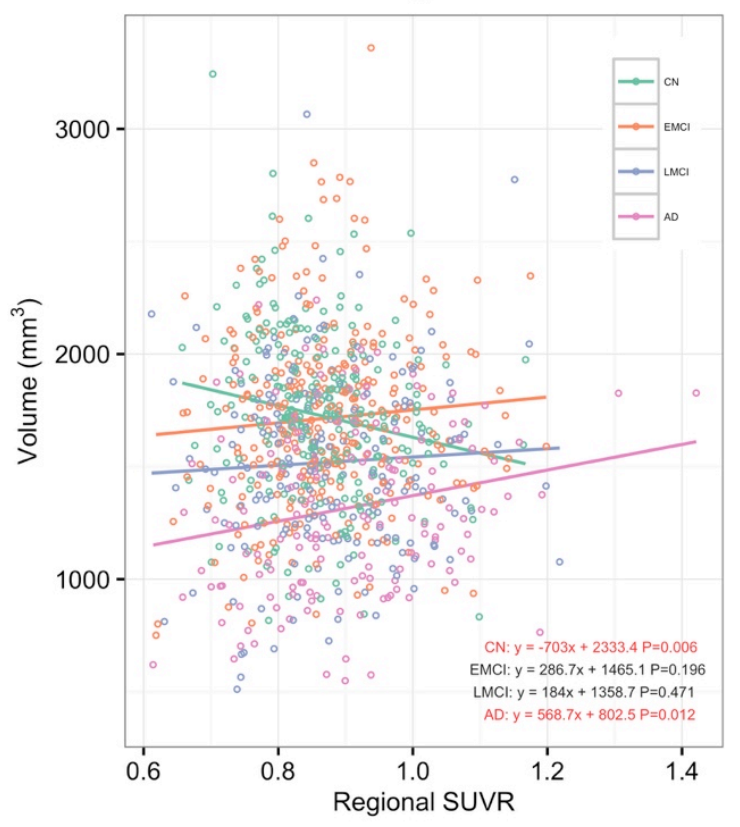

C

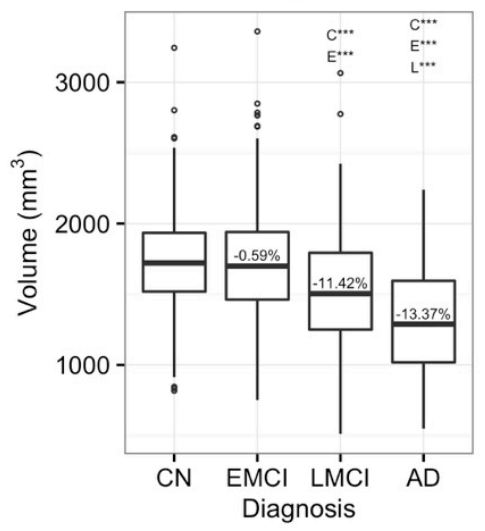

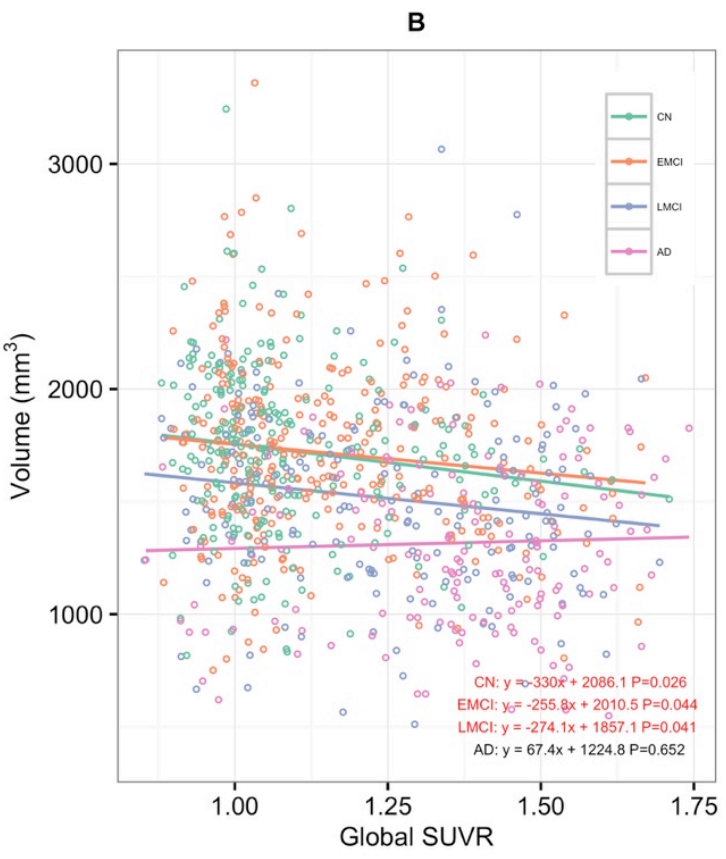

D

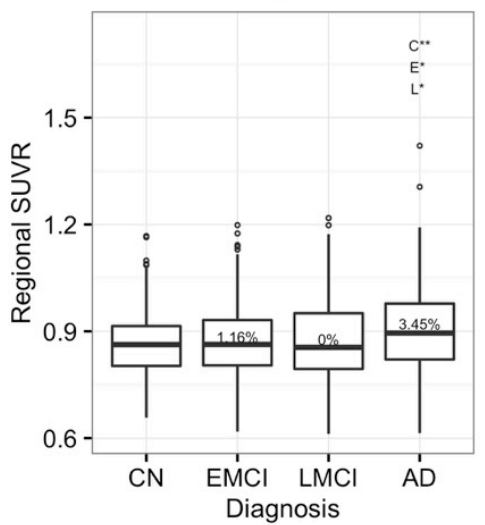

E

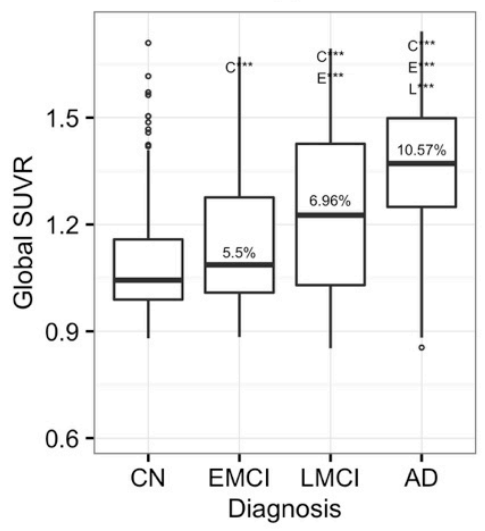

Fig. 16. Linear regression models and performance of volume, regional $A \beta$ load and global $A \beta$ load in right entorhinal. (A) Scatterplot of the regional A $\beta$ load and volume. The fitted lines are from linear regression models for each diagnosis stage, with formulas listed at the right bottom as well as the $\mathrm{p}$ values for regional $A \beta$ standardized uptake value ration (SUVR) in the linear regression models. (B) Scatterplot and the linear regression models of global $\mathrm{A} \beta$ load and regional volume. (C) Boxplot of volumes across all diagnostic stages: CN, EMCI, LMCI and AD. C, E, and L above the boxes represented a significant difference in the volume from CN, EMCI, LMCI group, respectively along with the significant level: ${ }^{*} \mathrm{p}<.05 ;{ }^{*} \mathrm{p}<.01$; $* * * p<.001$. Values in the box indicated the mean volume change rate from the previous stage. (D) Boxplot of regional A $\beta$ load across 4 diagnostic stages, represented by the regional A $\beta$ SUVR. (E) Boxplot of global A $\beta$ load across 4 diagnostic stages, represented by the whole cortical A $\beta$ SUVR. 
In addition, the $\mathrm{A} \beta \mathrm{SUVR}$ in right entorhinal (0.876 0.11, mean SD), left temporal pole (0.957 0.16) and in right parahippocampal $(0.9900 .12)$ was lower than that of the global (1.189 0.21), as well as the left inferior temporal (1.144 0.22), right precuneus (1.283 $0.27)$ and left inferior parietal (1.255 0.26) ROIs.

\subsection{Discussion}

There are two major findings in this study: (i) the association patterns of regional A $\beta$ load were generally consistent with and even more robust than the global A $\beta$ load for almost all cortical regions, except for entorhinal, temporal pole and parahippocampal, the three most AD-vulnerable regions [129-132]. (ii) A $\beta$ accumulation in those three high ADvulnerable regions showed sharp growth prior to the global A $\beta$ load reaching an abnormal level, but the speed slowed down after that. On the other hand, in other brain areas, the regional $A \beta$ load showed consistent increasing rate regardless of the global $A \beta$ burden.

The observed negative associations of cortical atrophy and global A $\beta$ load are consistent with previous findings $[121,127,133,134]$. The A $\beta$-related cortical atrophy was first detected in the EMCI stage in the following regions: parahippocampal [134], inferior parietal, precuneus, isthmus cingulate, supramarginal and insula, followed by entorhinal, temporal pole, fusiform, inferior temporal, posterior cingulate in the LMCI stage [127, 134], and significantly presented in rostral middle frontal in the AD stage ultimately. The regional-specific association across diagnosis stages and the enhanced correlation of $A \beta$ load with cortical atrophy in LMCI and AD stages agreed with the spatial topography of 
neurodegenerative change [79] and were also in line with the view that different brain areas can be at different atrophy levels regarding reactivity to $A \beta$ burden [10]. In addition, only right parahippocampal showed A $\beta$-load-related atrophy in EMCI stage. Also, precuneus presented such correlation firstly in right hemisphere in EMCI stage and extended to left hemisphere later in the LMCI stage. Such different patterns of left and right hemisphere supported the view of laterality in alterations [135].
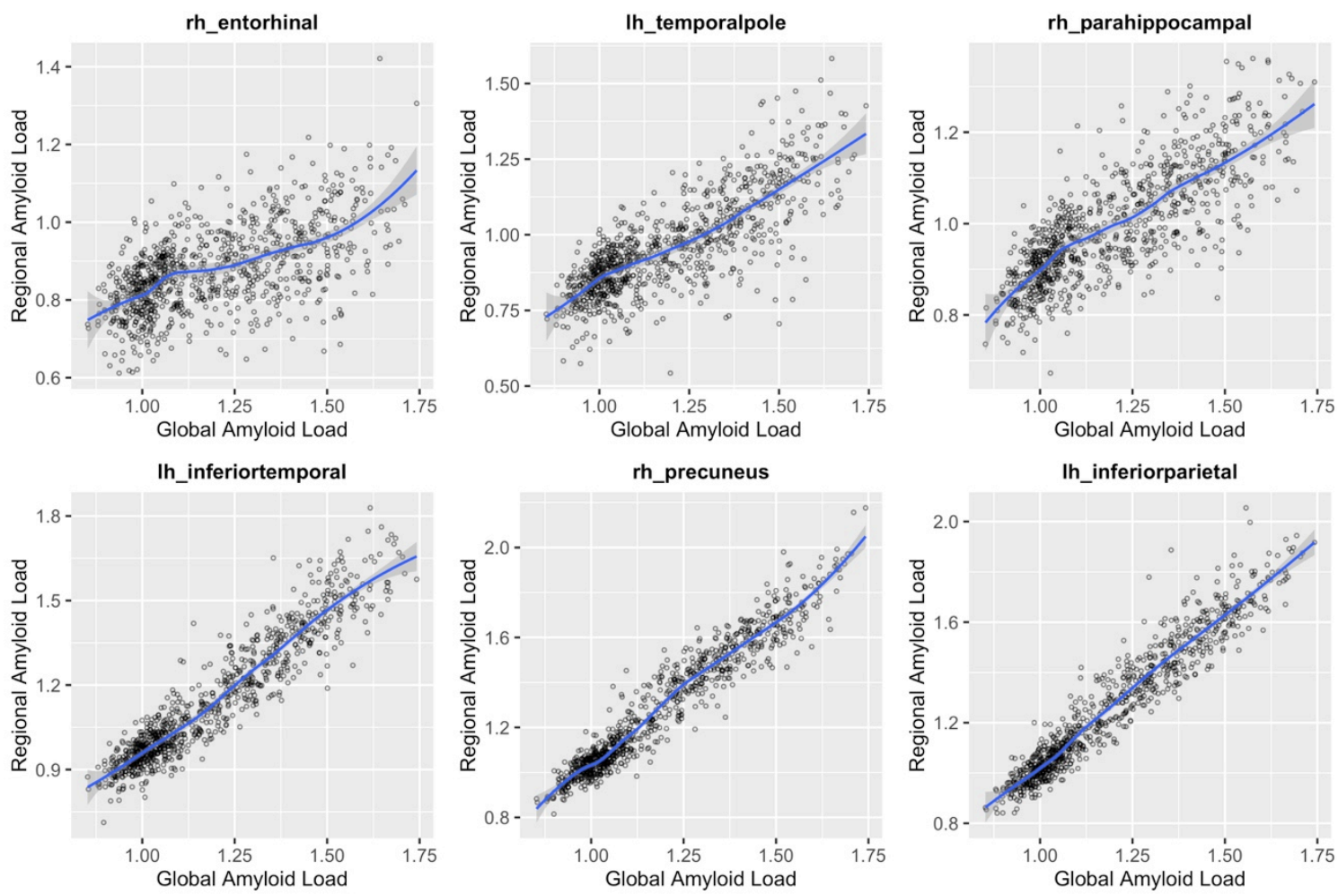

Fig. 17. Associations between regional and global $A \beta$ load. The fitted curves are from LOESS regression models for all subjects, with smoothing degree equals to 0.4 .

The inconsistent positive correlation of cortical atrophy with regional $\mathrm{A} \beta$ burden in right parahippocampal in CN stage had been reported in a previous study [136] that informed larger hippocampal and parahippocampal volume in $\mathrm{CN}$ participants with high $\mathrm{A} \beta$ load 
measured by PIB PET. Such hippocampal hypertrophy in the brains of normal elderly with $A \beta$ plaques compared to those without $A \beta$ plaques had been evidenced at some autopsy studies and were explained as the early response to the toxic effects of $A \beta$ peptide $[137,138]$. Besides, the larger cortical volume in EMCI stage followed by cortical atrophy in LMCI and AD stages in parahippocampal (Fig. 15C) and in precuneus (Fig. 16C) ROIs was also agreed with the view of biphasic trajectory of brain structure changes as reported in $[139,140]$, and may also be interpreted as neuronal hypertrophy and/or inflammatory response to the toxic effect of $A \beta$ peptide.

On the other hand, the negative correlation between regional/global $\mathrm{A} \beta$ burden and regional volume in $\mathrm{CN}$ stage was found only in right entorhinal ROI from the linear regression model (Fig. 16A), which was consistent with other studies [80, 88, 129, 130, 141] and supported the view that $A \beta$ deposition is associated with a pattern of cortical atrophy prior to the development of cognitive impairment, i.e., cognitive reserve [80]. This finding also confirmed that the entorhinal is one of the earliest brain regions revealing degeneration [142]. However, when accounting for the effects of age, APOE4 and ICV, such regional $\mathrm{A} \beta$-induced cortical atrophy in right entorhinal in $\mathrm{CN}$ stage was not detectable, instead, positive association was shown in EMCI stage and later in $\mathrm{AD}$ stage. A few previous studies had also reported increased metabolic activity, which is another way to express neurodegeneration, in the brain measured by FDG PET in MCI $[143,144]$ and increased cerebral blood flow in $\mathrm{AD}[134]$ in relation to the increased $\mathrm{A} \beta$ load. Such positive association may either reflect early response to the $A \beta$ toxic effect, or imply that $A \beta$ accumulation itself results from the increased neural activity $[134,143$, 
145]. In fact, we can see from Fig. 16 that both volume and $A \beta$ burden underwent significant change in $\mathrm{AD}, 13.37 \%$ atrophy compared with $\mathrm{LMCI}$ and $3.45 \% \mathrm{~A} \beta$ accumulation. Furthermore, the relatively unchanged $\mathrm{A} \beta$ load from $\mathrm{CN}$ to LMCI stage (1.16\% form CN to EMCI and 0\% form EMCI to LMCI, Fig. 4D) and the mild but not significant decreased volume size from $\mathrm{CN}$ to EMCI (-0.59\%) followed by the dynamically volume shrinking from EMCI to LMCI (-11.42\%) (Fig. 16C) inferred the postponed effect of $A \beta$ load on neurodegeneration [10].

The LOESS regression analyses of regional $A \beta$ load and global $A \beta$ accumulation also elicited the inconsistent patterns of these $3 \mathrm{AD}$ prone ROIs: entorhinal, temporal pole and parahippocampal. Unlike other brain areas where regional $A \beta$ accumulation was consistent with that in the whole cortical, $A \beta$ in these 3 ROIs underwent dynamically accumulate among patients identified as $\mathrm{A} \beta$ negative (AM-), considering the common cutoff value range of 1.10 to 1.12 [81, 82, 146, 147], and relatively slower increase for $\mathrm{A} \beta$ positive $(\mathrm{AM}+)$ patients. In fact, these 3 ROIs are known to be selectively vulnerable to pathology and undergo neurodegeneration before all other brain regions when exposed to toxins, including $\mathrm{A} \beta$. Once neurodegeneration begins in the entorhinal and the parahippocampal (Fig. 16C \& Fig. 16C), it seems to spread, like an infection, in a stereotyped fashion along certain pathways leading to neurodegeneration in the hippocampus, and then onto the posterior cingulate/precuneus (Fig. 15C) regions and later to the rest of the temporal, parietal and frontal neocortex. This finding suggests that $A \beta$ load in the entorhinal, temporal pole and parahippocampal is possibly the most important biomarkers to early detection of the disease. Also, biologically important cut 
off for AM+ vs. AM- should not only be the global cortical A $\beta$ SUVR, which most researchers are using, but entorhinal, temporal pole and parahippocampal SUVR should also be taken into consideration, even though their $A \beta$ levels happen to be much lower than the neocortical $A \beta$ level.

To conclude, the present study provided strong evidence for the effect of A $\beta$ load in entorhinal, temporal pole and parahippocampal on brain atrophy prior to other brain regions and to the global $A \beta$ load, suggesting the use of $A \beta$ load in these regions to assist early detection of $\mathrm{AD}$, and to determine the $\mathrm{A} \beta$ positivity rather than the global $\mathrm{A} \beta$ load. Further longitudinal analysis is needed to better understand the predictive value of $A \beta$ load within those AD prone regions. 


\title{
CHAPTER VI
}

\section{PATTERN ANALYSIS OF THE INTERACTION OF REGIONAL AMYLOID, CORTICAL THICKNESS, AND APOE GENOTYPE IN THE PROGRESSION OF ALZHEIMER'S DISEASE}

\begin{abstract}
6.1. Goal
Deposition of beta amyloid protein $(\mathrm{A} \beta)$ is known to be an early event that is closely associated with the pathogenesis of Alzheimer's disease (AD), along with related downstream events such as neuronal loss, neurofibrillary tangles, cortical thinning and cognitive deficits. APOE e4 allele (E4) is also known to be associated with increased risk for $\mathrm{AD}$.
\end{abstract}

The goal of this study is to examine the association of $A \beta$ deposition to cortical thickness (CTh), in healthy control (CN), early MCI (EMCI), late MCI (LMCI) and AD stages by controlling for E4 load, both in regional and hemispheric levels, and to interpret patterns of different brain regions based on their correlation performance among the four groups.

\subsection{Materials and Methods}

\subsubsection{Study Participants}

Data and image processing method used in the preparation of this study is the same as in the chapter II. 


\subsubsection{Statistical analysis}

To compare the subject's characteristics among the diagnostic groups (CN, EMCI, LMCI and $\mathrm{AD}$ ), analysis of variance (ANOVA) was implemented for age as well as for year of education, while Chi-square test was used for gender and E4 allele. Since only the significant difference of age was investigated, analysis of covariance (ANCOVA) adjusted for age was then introduced to compare the cognitive tests (MMSE and RAVLT) among groups.

Considering the mediated performance of E4 on the aggregated forms of $A \beta[148]$, we used Pearson partial correlation (PPC) controlling for the number of APOE e4 alleles to assess the relationship between regional as well as hemispheric CTh and SUVR, which was then used as the performance measure of such region in the specific groups $(\mathrm{CN}$, EMCI, LMCI or AD) later. Multiple-comparison correction was then considered across all PPC analyses by controlling false discovery rate (FDR). The statistical analysis was performed using R software ( $R$ 3.3.0) using the default significant level ( $p$ value) of 0.05 .

\subsubsection{Clustering Analysis}

To interpret patterns of ROIs based on their correlation performance, the complete linkage hierarchical clustering analysis (CL_HCA) was applied. The CL_HCA is carried out through a series of successive mergers. As is shown in Fig. 18, initially, there are as many clusters as objects, i.e. 5 in this case. The most similar objects are first merged as a 
group, i.e. G1 with objects a and b, and such groups are then merged according to their similarities. All subgroups are fused into a single cluster eventually.

In terms of how the complete linkage clustering proceeds, the similarity between clusters is determined by the distance (similarity) between the two elements, one from each cluster, that are most distant. The complete linkage function, i.e. distance $\mathrm{D}(\mathrm{U}, \mathrm{V})$ between clusters $\mathrm{U}$ and $\mathrm{V}$, is described in (6.1).

$$
D(U, V)=\max _{u \in U, v \in V} d(u, v)
$$

where $d(u, v)$ is the distance between $u$ and $v$, which are the objects in cluster $\mathrm{U}$ and cluster $\mathrm{V}$, respectively.

\section{Cluster Dendrogram}
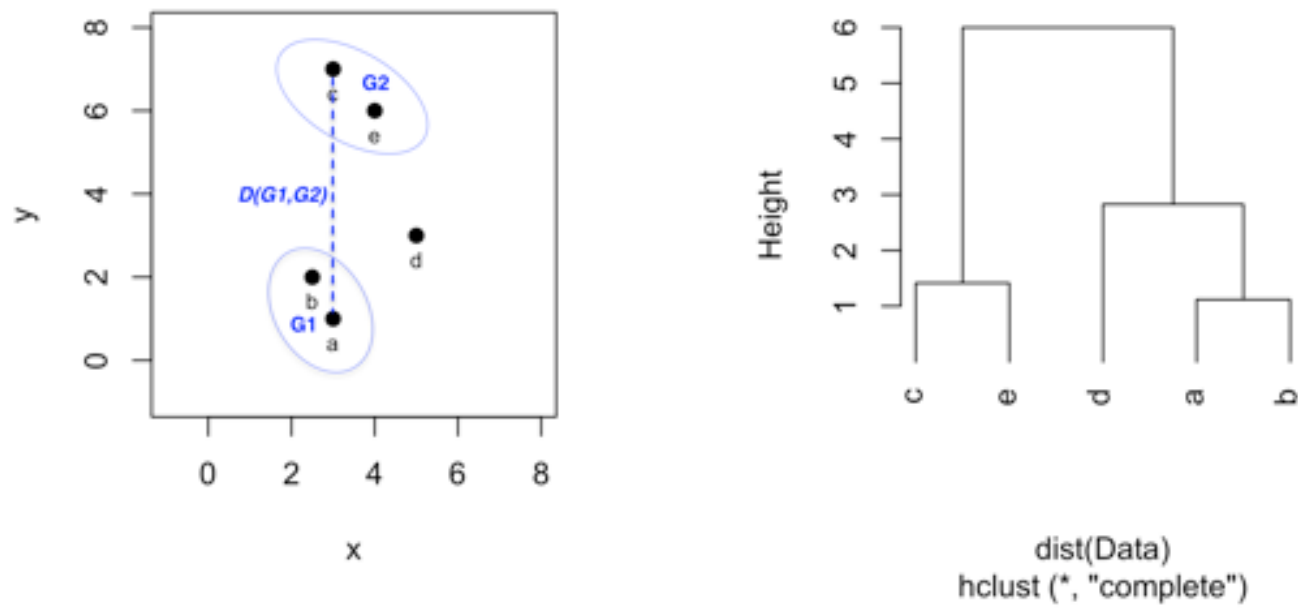

Fig. 18. Complete linkage hierarchical clustering illustration: (a) similarity. The distance of new cluster G1 and $\mathrm{G} 2$ is defined as $\mathrm{d}(\mathrm{a}, \mathrm{c})$ which is the maximum distance among $(\mathrm{a}, \mathrm{c}),(\mathrm{a}, \mathrm{e}),(\mathrm{b}, \mathrm{c})$ and $(\mathrm{b}, \mathrm{e})$; $(\mathrm{b})$ dendrogram. Object $\mathrm{a}$ and $\mathrm{b}$ are fist merged as new cluster G1, followed by object $\mathrm{c}$ and e (G2), then object $\mathrm{d}$ and cluster G1 were merged as new cluster, and finally merged with G2 into a single cluster. 
In the context of this study, the $u$ and $v$ represent the specific ROIs in each cluster, and the region-to-region distance $d(u, v)$ is as defined in (6.2).

$$
d(u, v)=\sqrt{\left(r_{u}^{C N}-r_{v}^{C N}\right)^{2}+\left(r_{u}^{E M C I}-r_{v}^{E M C I}\right)^{2}+\left(r_{u}^{L M C I}-r_{v}^{L M C I}\right)^{2}+\left(r_{u}^{A D}-r_{v}^{A D}\right)^{2}}
$$

where, $r_{u}^{C N}$ represents the PPC coefficient (5.2) of region $\mathrm{u}$ in group CN. For those not showing significant correlation, the PC coefficient $r$ is set to 0 .

Both the correlation results and clustering analysis are represented using heatmaps to facilitate both visualization and interpretation of the results provided in the next section.

\subsection{Results}

At the regional level, 25 out of 68 ROIs presented significant correlations of SUVR and CTh at least in one of the four diagnostic groups (CN, EMCI, LMCI, and AD), among which, 9 in bilateral hemispheres (bi_), 3 in left hemisphere (lh_), and 4 in right hemisphere ( $\mathrm{rh} \_$, respectively. While at the hemispheric level, both mean hemisphere cortical thickness showed significant negative associations to the corresponding mean $\mathrm{A} \beta$ deposition of left hemisphere but only in the EMCI stage (Fig. 19).

In the pattern of such relationship performance among the 4 groups, 6 main clusters of those 25 ROIs were identified:

- $\mathrm{C} 1$ (negative $\mathrm{CN}+\mathrm{EMCI})$ :

○ rh_inferior_temporal

- $\mathrm{C} 2$ (negative EMCI): 
- bi_fusiform, bi_precuneus, bi_superior_temporal, bi_lateral_occipital

o lh_medialorbitofrontal, $\mathrm{lh} \_$isthmuscingulate

○ rh_superamarginal, rh_middle_temporal

- $\mathrm{C} 3$ (negative EMCI+LMCI):

- lh_inferior_temporal, lh_middle_temporal,

lh_banks_of_the_Superior_semporal_sulcus (lh_bankssts)

$\circ \quad$ rh_inferior_parietal

- C4 (negative LMCI):

○ lh_inferior_parietal

- $\mathrm{C} 5$ (positive $\mathrm{CN}$ ):

○ rh_postcentral, rh_transversetemporal, rh_precentral

- C6 (positive AD):

○ bi_entorhinal, bi_temporal_pole

Specifically, ROIs in $\mathrm{C} 1$ exhibited steady negative significant correlation trends among $\mathrm{CN}$ and EMCI groups; $\mathrm{C} 2$ showed reversed correlation in EMCI stage; C3 showed such reversed relationship also in EMCI stage, which were maintained in the LMCI stage; C4 showed negative correlation only in the LMCI stage. On the other hand, ROIs in C5 presented positive significant correlation in $\mathrm{CN}$ stage; $\mathrm{C} 6$ showed such correlation in the AD stage. Similar associations were found even when using Pearson correlation (Fig. 20). 


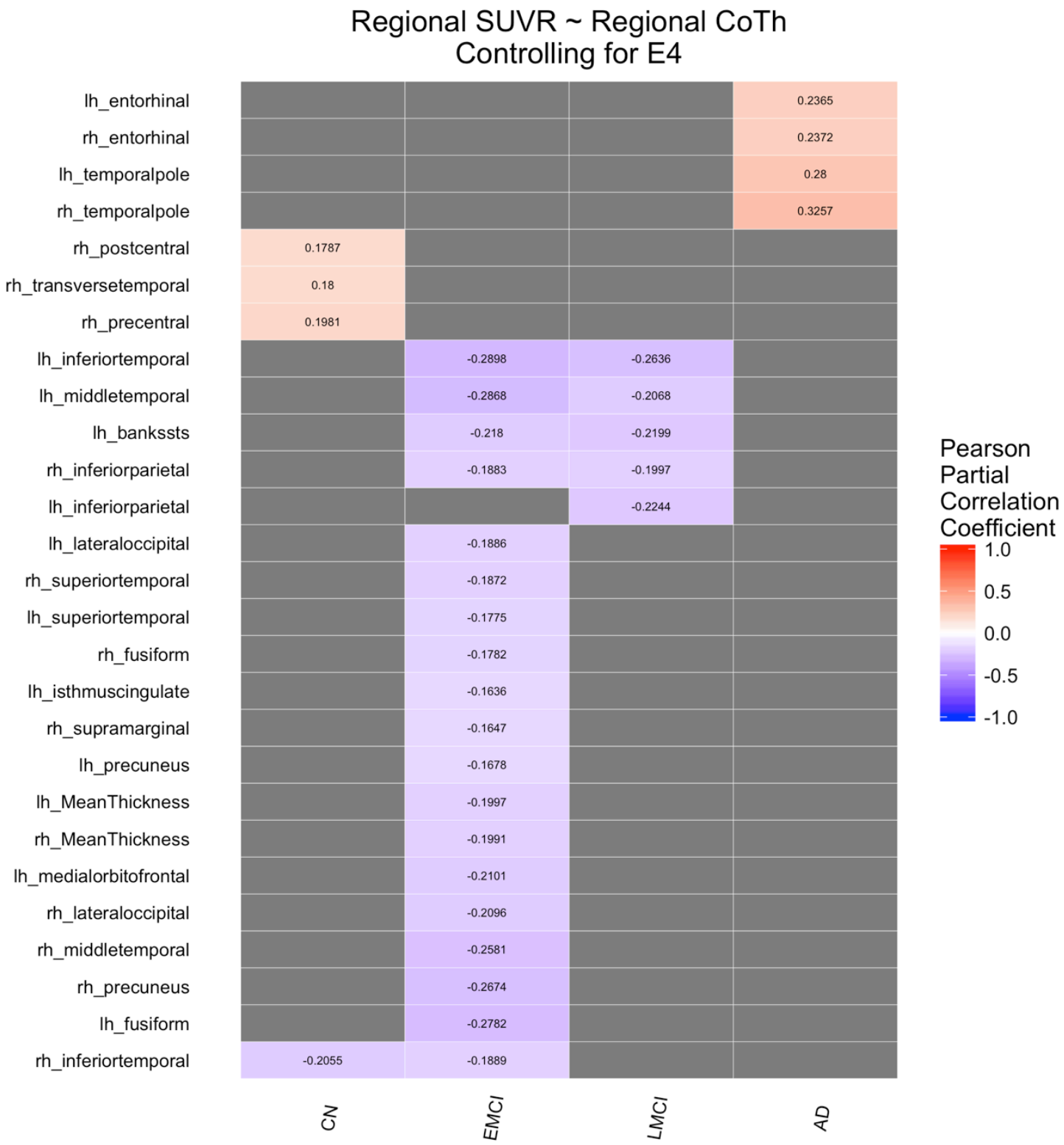

Fig. 19. Heatmap representation of clustering analysis. The PPC coefficient, i.e., $r$ value, is shown in each block.

The remaining 43 ROIs, which were found to be without any significant association, are listed below: 
- Bilateral: bi_frontal_pole,bi_superior_frontal, bi_rostral_middle_frontal, bi_pars_triangularis, bi_pars_orbitalis, bi_paracentral, bi_lingual, bi_lateral_orbitofrontal, bi_insula bi_cadual_middle_frontal, bi_cuneus bi_caudal_anterior_cingulate, bi_rostral_anterior_cingulate, bi_posterior_cingulate, bi_superior_parietal, bi_parahippocampal, bi_pericalarine,

- Left: lh_precentral, lh_postcentral, lh_superamarginal, lh_transverse_temporal

- Right: rh_medial_orbitofrontal, rh_bankssts, rh_isthmus_cingulate

\subsection{Discussion and Conclusion}

The main findings of this study suggest that there are cortical areas that have significant association with $A \beta$ deposition, while other regions seem to be relatively independent from $A \beta$ burden. Also, patterns of those regions showing significant association are in general distinctive with regards to the different stages of the disease, with some showing relative similarities. Briefly, negative associations, i.e. higher $A \beta$ deposition is associated with reduced cortical thickness, were detected mainly among EMCI with some lingering into LMCI stage; whereas positive correlations, i.e. higher $\mathrm{A} \beta$ deposition is related to higher cortical thickness were shown at the $\mathrm{CN}$ and $\mathrm{AD}$ stages.

Three ROIs in right hemisphere showed positive correlation in CN stage, however, such correlation did not exist later for the EMCI and subsequent stages, which could indicate that the amyloid deposition in those regions increased significantly in the EMCI stage (Fig. 20a). 
A
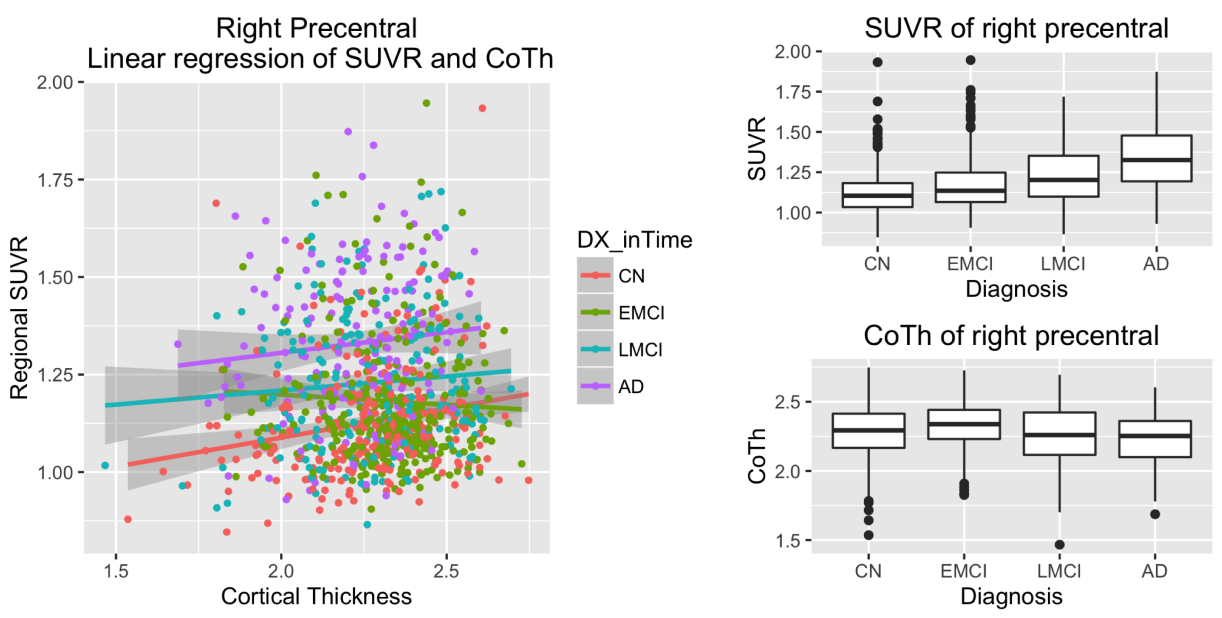

B
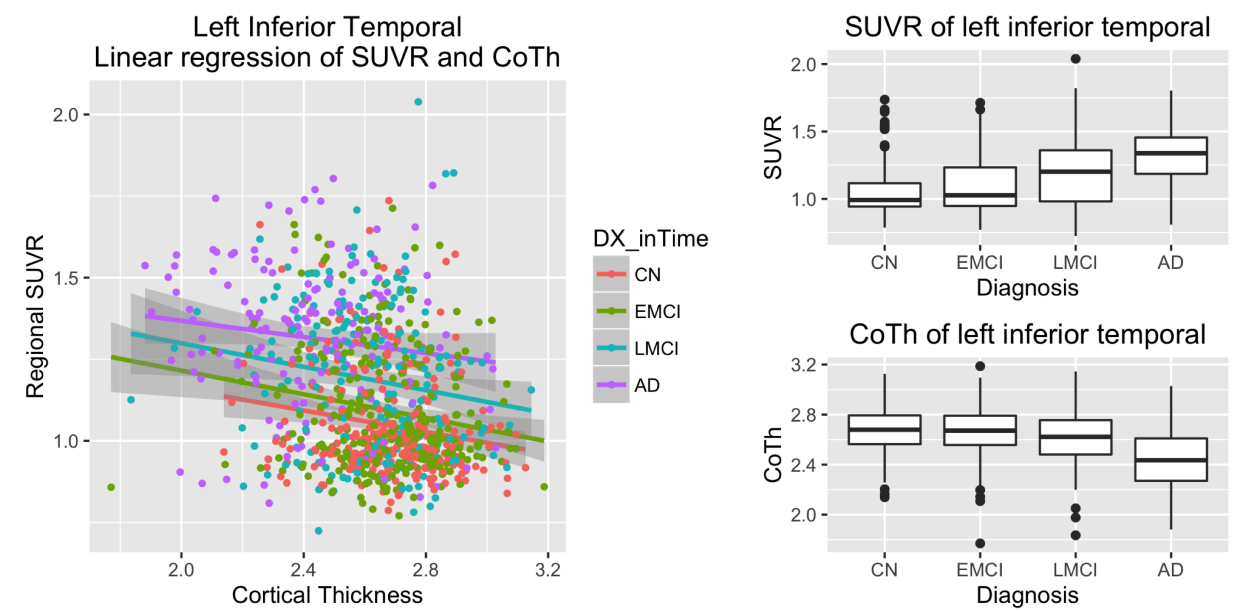

C

Right Entorhinal
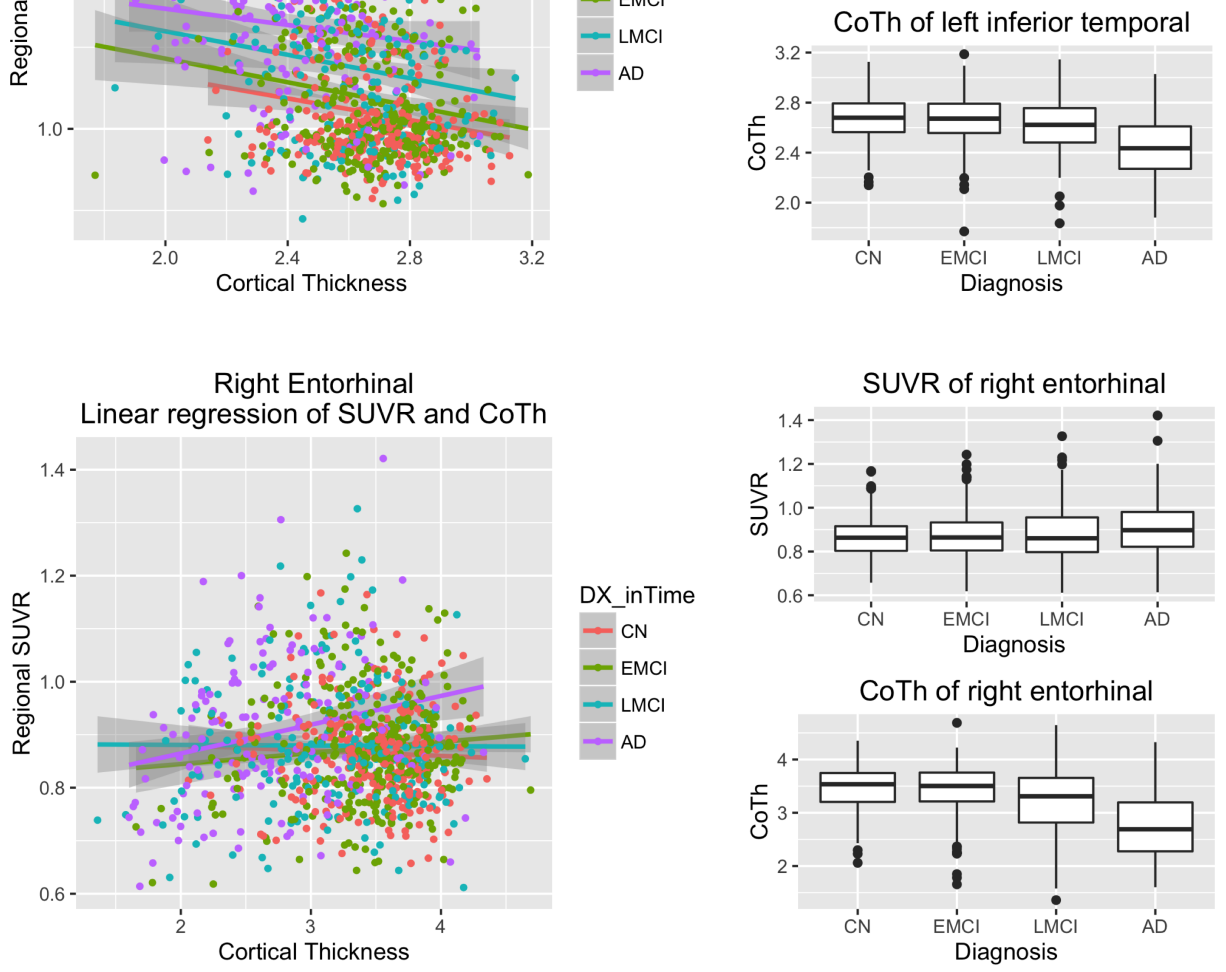

Fig. 20. Scatterplot, linear regression as well as boxplot of (a) right precentral, (b) left inferior temporal, and (c) right entorhinal. 
Almost all ROIs showing negative association appeared in the EMCI stage, except for the left inferior parietal. Four of them persisted with such trend towards the LMCI stage, implying that both $\mathrm{A} \beta$ load and cortical thinning are active in $\mathrm{MCI}$ regions, i.e., more $\mathrm{A} \beta$ load exists in direct association with more brain atrophy [119-126](Fig. 20b). Bilateral entorhinal and temporal pole, which are well-known vulnerable ROIs, showed positive correlation in the $\mathrm{AD}$ stage, whereas no correlation was found in other stages, which may be due to greater regional atrophy and relatively stable amyloid depositions in such regions, i.e., the increasing rate of $A \beta$ deposition is low compared with the greater regional atrophy (Fig. 20c). Also, from Fig. 20, we can see that the mean CTh of EMCI patients is thicker than that of CN, same as SUVR, which may also be the reason of no significant negative correlation being detected in such vulnerable regions as the entorhinal and temporal pole.

In retrospect, the cortical regions can be clustered into 2 general groups, positive correlation in $\mathrm{CN}$ or $\mathrm{AD}$, and negative correlation in $\mathrm{EMCI}$ and/or LMCI, and 6 more specific groups were then recognized, suggesting the merits of analyzing the regional interplay that exists between $A \beta$ deposition with cortical thickness at the different stages of Alzheimer's disease. 


\section{CHAPTER VII}

\section{REGIONALSHIP BETWEEN REGIONAL CORTICAL THICKNESS, AMYLOID LOAD AND SELECTIVE VULNERABILITY TO ATROPHY IN ALZHEIMER'S \\ DISEASE}

\subsection{Goal}

CTh is known to vary greatly across different brain regions, with primary motor and sensory cortices being thinner than association cortices, although the effect of normal aging and disease may alter this relationship [149]. Thinning of the cortex between young and middle-aged adults was found to be greatest in heteromodal association cortex and regions of high postnatal surface area [150]. Some studies have shown that temporal and occipital regions have less cortical thinning than parietal and frontal cortices, and there may even be age-related thickening of the ERC among older cognitively normal individuals [149].

In this study, we examined relationships between baseline regional $\mathrm{CTh}\left(\mathrm{rCTh}^{\mathrm{CN}}\right)$, baseline regional $\mathrm{A} \beta$ load $\left(\mathrm{rSUVR} \mathrm{R}^{\mathrm{CN}}\right.$ ) and the severity of change in $\mathrm{CTh}$ between $\mathrm{CN}$ subjects and $\mathrm{AD}$ patients $\left(\% \mathrm{CTh}^{\text {Diff }}\right)$, with consideration of the effect of APOE4 carrier status and global A $\beta$ load. 


\subsection{Materials and Methods}

\subsubsection{Study participants}

In this study, we focused on subjects classified as cognitively normal (CN) or AD by ADNI, who underwent MRI and AV45 PET scans within 6 months. Since only few CN individuals have progressed to $\mathrm{AD}$ dementia in the ADNI cohort, we matched the $\mathrm{AD}$ patients to $\mathrm{CN}$ subjects based on the age, gender and the APOE4 status. Subjects without APOE4 alleles were classified as APOE4 negative (E4-), while those with one or more APOE4 allele were classified as APOE4 positive (E4+). Overall, $105 \mathrm{CN}$ subjects and 105 AD patients were considered. Table 20 provides the demographic characteristics of the participants.

Table 20. Age, gender-matched participants' demographic information

\begin{tabular}{llll}
\hline & CN & AD & P value $^{\mathbf{a}}$ \\
\hline $\mathbf{N}$ & 105 & 105 & 1 \\
Female/Male & $45 / 60$ & $45 / 60$ & 1 \\
E4-/+ & $47 / 58$ & $47 / 58$ & 1 \\
gSUVR-/+ & $65 / 40$ & $22 / 83$ & $\mathbf{4 . 0 1 e - 0 9}$ \\
Age & $75.22(6.95)$ & $75.22(7)$ & 1 \\
Education & $16.34(2.55)$ & $16.14(2.64)$ & 0.5766 \\
CDR-SB & $0.06(0.24)$ & $4.61(1.81)$ & $<\mathbf{2 . 2 e - 1 6}$ \\
MMSE & $29.06(1.14)$ & $23.02(2.59)$ & $<\mathbf{2 . 2 e - 1 6}$ \\
\hline
\end{tabular}

${ }^{a}$ P-values are for t-test (continuous variables: Age, Education, Clinical Dementia Rating Scale Sum of Boxes (CDR-SB) and Mini-Mental State Examination (MMSE)) and for Chi-square test (categorical variables: gender, APOE genotype (E4-/E4+) and global A $\beta$ load status (gSUVR-/gSUVR+)), The criteria for significance was set at level $\mathrm{p}<0.05$.

${ }^{\mathrm{b}}$ Values are represented as mean(SD) for all continuous attributes 


\subsubsection{Severity of Change in Cortical Thickness Between CN and AD Patients}

Once the MRI and PET were processed as descripted in Chater II, we got the regional cortical thickness (rCTh), the regional amyloid load (rSUVR) and the gloal amyloid (gSUVR). A widely used threshold value of 1.11 is used to delineate $A \beta$ positive (gSUVR+) and $\mathrm{A} \beta$ negative (gSUVR-) status.

The percent difference $\mathrm{CTh}$ between $\mathrm{CN}$ and $\mathrm{AD}$ subjects $\left(\% \mathrm{CTh}^{\text {Diff }}\right)$ for those with and without the E4 allele was calculated using (7.1) for each of 68 brain regions separately.

$$
\% C T h^{D i f f}=\left(r C T h^{C N}-r C T h^{A D}\right) / r C T h^{C N} \times 100
$$

where $\mathrm{rCTh}^{\mathrm{CN}}$ and $\mathrm{rCTh}^{\mathrm{AD}}$ are the mean $\mathrm{CTh}$ among $\mathrm{CN}$ individuals and $\mathrm{AD}$ patients, respectively. Taking the $\mathrm{CN}$ with $\mathrm{E} 4+$ group for example, the mean $\mathrm{rCTh}$ for a specific region was measured as (7.2):

$$
r C T h^{C N}=\sum_{i=1}^{N_{C N E 4+}} r C T h_{i} / N_{C N E+}
$$

where $\mathrm{N}_{\mathrm{CNE} 4+}$ is the number of $\mathrm{CN}$ subjects with $\mathrm{E} 4+$ and $\mathrm{rCTh}_{\mathrm{i}}$ is the $\mathrm{CTh}$ of the specific region for subject $\mathrm{i}$.

Similar calculations were performed for $\mathrm{A} \beta$ positive $(\mathrm{gSUVR}+)$ and $\mathrm{A} \beta$ negative (gSUVR-) subjects.

\subsubsection{Statistical analysis}

To compare subjects' characteristics between $\mathrm{CN}$ and AD, a series of Student's t-tests were employed for age, years of education, as well as for the cognitive tests that include 
Mini-Mental State Examination (MMSE) and Clinical Dementia Rating-Sum of Boxes (CDR-SB); while the Chi-square test was used to account for gender, APOE4 status and global amyloid load status.

The association between \%CThDiff and $\mathrm{rCThCN}$ across 68 cortical regions was examined using the linear regression, as well as Pearson correlation, separately for E4+ and E4- participants. The comparative strength of the magnitude between these correlations was examined using the two-tailed Fisher's z transformation.

A similar set of procedures was applied to examine the magnitude of correlations between gSUVR+ and gSUVR-.

The potential effect of rSUVRCN on the relationship between $\mathrm{rCThCN}$ and \%CThDiff was evaluated by employing the Pearson Partial Correlation and multiple linear regression models with simultaneous adjustment of predictors, i.e. $\mathrm{rCThCN}$ and rSUVRCN.

All statistical analyses were performed using R software (R 3.3.3) [83] and the statistical significance level was set at 0.05 .

\subsection{Results}

As shown in Table 20, there was no significant difference between $\mathrm{CN}$ and AD subjects, with regards to age, gender, the frequency of E4+ or years of education. However, the 
frequency of gSUVR+ was higher among AD than CN subjects. Table 21 and Table 22 provide the mean regional $\mathrm{CThs}$ for the $\mathrm{CN}$ and $\mathrm{AD}$ groups, \%CThDiff and the rSUVRCN in the left hemisphere for E4+ and E4- subjects, respectively.

\subsubsection{Associations between rCThCN and Regional \%CThDiff for E4+ and E4- Subjects}

As can be observed from Table 23 and Fig. 21, results indicate that greater $\mathrm{rCThCN}$ is associated with greater \%CThDiff, both before $(\mathrm{r}=0.639$, $\mathrm{p}$-value $<0.001$ for $\mathrm{E} 4+; \mathrm{r}=$ $0.768, \mathrm{p}<0.001$ for E4-) and after $(\mathrm{r}=0.521, \mathrm{p}<0.001$ for $\mathrm{E} 4+; \mathrm{r}=0.694, \mathrm{p}<0.001$ for E4-) adjusting for the effect of rSUVRCN.

We compared the magnitude of the Pearson correlation coefficients for E4+ versus E4groups using Fisher's z-transformation. Results showed no difference in the magnitude of the correlation coefficient between the E4+ and E4- groups $(z=-1.469 ; \mathrm{p}=0.142)$. 
Table 21. CTh in the $\mathrm{CN}$ and AD Groups, \% mean differences between $\mathrm{CN}$ and $\mathrm{AD}$ groups (\% $\mathrm{CTh}^{\mathrm{Diff}}$ ) and regional $\mathrm{A} \beta$ load $\left(\mathrm{rSUVR}^{\mathrm{CN}}\right)$, for $\mathrm{E} 4+$ subjects in the left hemisphere

\begin{tabular}{|c|c|c|c|c|}
\hline \multirow[t]{2}{*}{ ROI } & \multicolumn{4}{|c|}{ E4+ } \\
\hline & $\mathrm{rCTh}^{\mathrm{CN}}$ & $\mathrm{rCTh}^{\mathrm{AD}}$ & $\% \mathrm{CTh}^{\text {Diff }}$ & rSUVR $^{\mathrm{CN}}$ \\
\hline entorhinal & $3.38(.35)^{\mathrm{a}}$ & $2.60(.47)$ & $23.08 \%$ & $0.89(.08)$ \\
\hline parahippocampal & $2.69(.35)$ & $2.31(.37)$ & $14.13 \%$ & $0.96(.11)$ \\
\hline temporalpole & $3.58(.31)$ & $3.11(.48)$ & $13.13 \%$ & $0.94(.14)$ \\
\hline middletemporal & $2.71(.17)$ & $2.44(.23)$ & $9.96 \%$ & $1.14(.21)$ \\
\hline superiortemporal & $2.59(.19)$ & $2.35(.19)$ & $9.27 \%$ & $1.11(.19)$ \\
\hline fusiform & $2.62(.17)$ & $2.38(.19)$ & $9.16 \%$ & $1.11(.15)$ \\
\hline inferiortemporal & $2.67(.16)$ & $2.44(.26)$ & $8.61 \%$ & $1.13(.20)$ \\
\hline isthmuscingulate & $2.36(.22)$ & $2.16(.20)$ & $8.47 \%$ & $1.24(.19)$ \\
\hline inferiorparietal & $2.21(.18)$ & $2.05(.19)$ & $7.24 \%$ & $1.23(.25)$ \\
\hline bankssts & $2.32(.17)$ & $2.16(.20)$ & $6.90 \%$ & $1.4(.24)$ \\
\hline precuneus & $2.15(.17)$ & $2.01(.15)$ & $6.51 \%$ & $1.25(.25)$ \\
\hline lateraloccipital & $2.02(.17)$ & $1.91(.19)$ & $5.45 \%$ & $1.17(.18)$ \\
\hline supramarginal & $2.34(.17)$ & $2.22(.15)$ & $5.13 \%$ & $1.19(.21)$ \\
\hline superiorparietal & $1.99(.17)$ & $1.89(.15)$ & $5.03 \%$ & $1.17(.21)$ \\
\hline insula & $2.93(.17)$ & $2.83(.18)$ & $3.41 \%$ & $1.15(.17)$ \\
\hline superiorfrontal & $2.49(.16)$ & $2.41(.17)$ & $3.21 \%$ & $1.15(.21)$ \\
\hline transversetemporal & $2.19(.28)$ & $2.12(.21)$ & $3.20 \%$ & $1.18(.20)$ \\
\hline caudalmiddlefrontal & $2.34(.18)$ & $2.28(.17)$ & $2.56 \%$ & $1.24(.20)$ \\
\hline posteriorcingulate & $2.40(.19)$ & $2.34(.17)$ & $2.50 \%$ & $1.30(.23)$ \\
\hline parsorbitalis & $2.54(.23)$ & $2.48(.25)$ & $2.36 \%$ & $1.09(.20)$ \\
\hline cuneus & $1.72(.14)$ & $1.68(.14)$ & $2.33 \%$ & $1.14(.14)$ \\
\hline rostralmiddlefrontal & $2.15(.15)$ & $2.1(.15)$ & $2.33 \%$ & $1.23(.25)$ \\
\hline medialorbitofrontal & $2.28(.17)$ & $2.23(.14)$ & $2.19 \%$ & $1.11(.22)$ \\
\hline lingual & $1.83(.14)$ & $1.79(.14)$ & $2.19 \%$ & $1.06(.11)$ \\
\hline postcentral & $1.87(.17)$ & $1.83(.14)$ & $2.14 \%$ & $1.09(.15)$ \\
\hline parsopercularis & $2.39(.14)$ & $2.34(.15)$ & $2.09 \%$ & $1.2(.20)$ \\
\hline lateralorbitofrontal & $2.48(.16)$ & $2.43(.19)$ & $2.02 \%$ & $1.19(.19)$ \\
\hline parstriangularis & $2.23(.18)$ & $2.19(.15)$ & $1.79 \%$ & $1.21(.22)$ \\
\hline precentral & $2.33(.22)$ & $2.29(.17)$ & $1.72 \%$ & $1.16(.13)$ \\
\hline caudalanteriorcingulate & $2.74(.33)$ & $2.71(.39)$ & $1.09 \%$ & $1.30(.21)$ \\
\hline rostralanteriorcingulate & $2.79(.31)$ & $2.76(.29)$ & $1.08 \%$ & $1.26(.24)$ \\
\hline frontalpole & $2.55(.27)$ & $2.56(.29)$ & $-0.39 \%$ & $1.01(.24)$ \\
\hline pericalcarine & $1.48(.14)$ & $1.49(.13)$ & $-0.68 \%$ & $1.27(.18)$ \\
\hline paracentral & $2.17(.17)$ & $2.19(.17)$ & $-0.92 \%$ & $1.19(.18)$ \\
\hline
\end{tabular}

${ }^{a}$ Values are represented as mean(sd). CTh unit: millimeter $(\mathrm{mm})$. 
Table 22. $\mathrm{CTh}$ in the $\mathrm{CN}$ and $\mathrm{AD}$ Groups, \% mean differences between $\mathrm{CN}$ and $\mathrm{AD}$ groups (\% $\mathrm{CTh}{ }^{\mathrm{Diff}}$ ) and regional $\mathrm{A} \beta$ load $\left(\mathrm{rSUVR}{ }^{\mathrm{CN}}\right)$, for E4- subjects in the left hemisphere

\begin{tabular}{|c|c|c|c|c|}
\hline \multirow[t]{2}{*}{ ROI } & \multicolumn{4}{|c|}{ E4- } \\
\hline & $\mathrm{rCTh}^{\mathrm{CN}}$ & $\mathrm{rCTh}^{\mathrm{CN}}$ & $\mathrm{rCTh}^{\mathrm{CN}}$ & $\mathrm{rCTh}^{\mathrm{CN}}$ \\
\hline entorhinal & $3.30(.33)^{\mathrm{a}}$ & $3.30(.33)$ & $3.30(.33)$ & $\overline{3.30(.33)}$ \\
\hline parahippocampal & $2.62(.38)$ & $2.62(.38)$ & $2.62(.38)$ & $2.62(.38)$ \\
\hline temporalpole & $3.52(.39)$ & $3.52(.39)$ & $3.52(.39)$ & $3.52(.39)$ \\
\hline middletemporal & $2.65(.21)$ & $2.65(.21)$ & $2.65(.21)$ & $2.65(.21)$ \\
\hline superiortemporal & $2.54(.23)$ & $2.54(.23)$ & $2.54(.23)$ & $2.54(.23)$ \\
\hline fusiform & $2.55(.18)$ & $2.55(.18)$ & $2.55(.18)$ & $2.55(.18)$ \\
\hline inferiortemporal & $2.63(.16)$ & $2.63(.16)$ & $2.63(.16)$ & $2.63(.16)$ \\
\hline isthmuscingulate & $2.27(.22)$ & $2.27(.22)$ & $2.27(.22)$ & $2.27(.22)$ \\
\hline inferiorparietal & $2.18(.18)$ & $2.18(.18)$ & $2.18(.18)$ & $2.18(.18)$ \\
\hline bankssts & $2.27(.20)$ & $2.27(.20)$ & $2.27(.20)$ & $2.27(.20)$ \\
\hline precuneus & $2.12(.19)$ & $2.12(.19)$ & $2.12(.19)$ & $2.12(.19)$ \\
\hline lateraloccipital & $1.95(.15)$ & $1.95(.15)$ & $1.95(.15)$ & $1.95(.15)$ \\
\hline supramarginal & $2.27(.19)$ & $2.27(.19)$ & $2.27(.19)$ & $2.27(.19)$ \\
\hline superiorparietal & $1.96(.20)$ & $1.96(.20)$ & $1.96(.20)$ & $1.96(.20)$ \\
\hline insula & $2.93(.19)$ & $2.93(.19)$ & $2.93(.19)$ & 2.93(.19) \\
\hline superiorfrontal & $2.46(.20)$ & $2.46(.20)$ & $2.46(.20)$ & $2.46(.20)$ \\
\hline transversetemporal & $2.13(.26)$ & $2.13(.26)$ & $2.13(.26)$ & $2.13(.26)$ \\
\hline caudalmiddlefrontal & $2.33(.20)$ & $2.33(.20)$ & $2.33(.20)$ & $2.33(.20)$ \\
\hline posteriorcingulate & $2.40(.18)$ & $2.40(.18)$ & $2.40(.18)$ & $2.40(.18)$ \\
\hline parsorbitalis & $2.52(.23)$ & $2.52(.23)$ & $2.52(.23)$ & $2.52(.23)$ \\
\hline cuneus & $1.68(.15)$ & $1.68(.15)$ & $1.68(.15)$ & $1.68(.15)$ \\
\hline rostralmiddlefrontal & $2.16(.16)$ & $2.16(.16)$ & $2.16(.16)$ & $2.16(.16)$ \\
\hline medialorbitofrontal & $2.29(.15)$ & $2.29(.15)$ & $2.29(.15)$ & $2.29(.15)$ \\
\hline lingual & $1.82(.13)$ & $1.82(.13)$ & $1.82(.13)$ & $1.82(.13)$ \\
\hline postcentral & $1.84(.17)$ & $1.84(.17)$ & $1.84(.17)$ & $1.84(.17)$ \\
\hline parsopercularis & $2.36(.19)$ & $2.36(.19)$ & $2.36(.19)$ & $2.36(.19)$ \\
\hline lateralorbitofrontal & $2.46(.15)$ & $2.46(.15)$ & $2.46(.15)$ & $2.46(.15)$ \\
\hline parstriangularis & $2.23(.18)$ & $2.23(.18)$ & $2.23(.18)$ & $2.23(.18)$ \\
\hline precentral & $2.3(.26)$ & $2.3(.26)$ & $2.3(.26)$ & $2.3(.26)$ \\
\hline caudalanteriorcingulate & $2.63(.33)$ & $2.63(.33)$ & $2.63(.33)$ & $2.63(.33)$ \\
\hline rostralanteriorcingulate & $2.81(.28)$ & $2.81(.28)$ & $2.81(.28)$ & $2.81(.28)$ \\
\hline frontalpole & $2.6(.29)$ & $2.6(.29)$ & $2.6(.29)$ & $2.6(.29)$ \\
\hline pericalcarine & $1.47(.14)$ & $1.47(.14)$ & $1.47(.14)$ & $1.47(.14)$ \\
\hline paracentral & $2.16(.23)$ & $2.16(.23)$ & $2.16(.23)$ & $2.16(.23)$ \\
\hline
\end{tabular}

${ }^{\mathrm{a}}$ Values are represented as mean(sd). CTh unit: millimeter $(\mathrm{mm})$. 


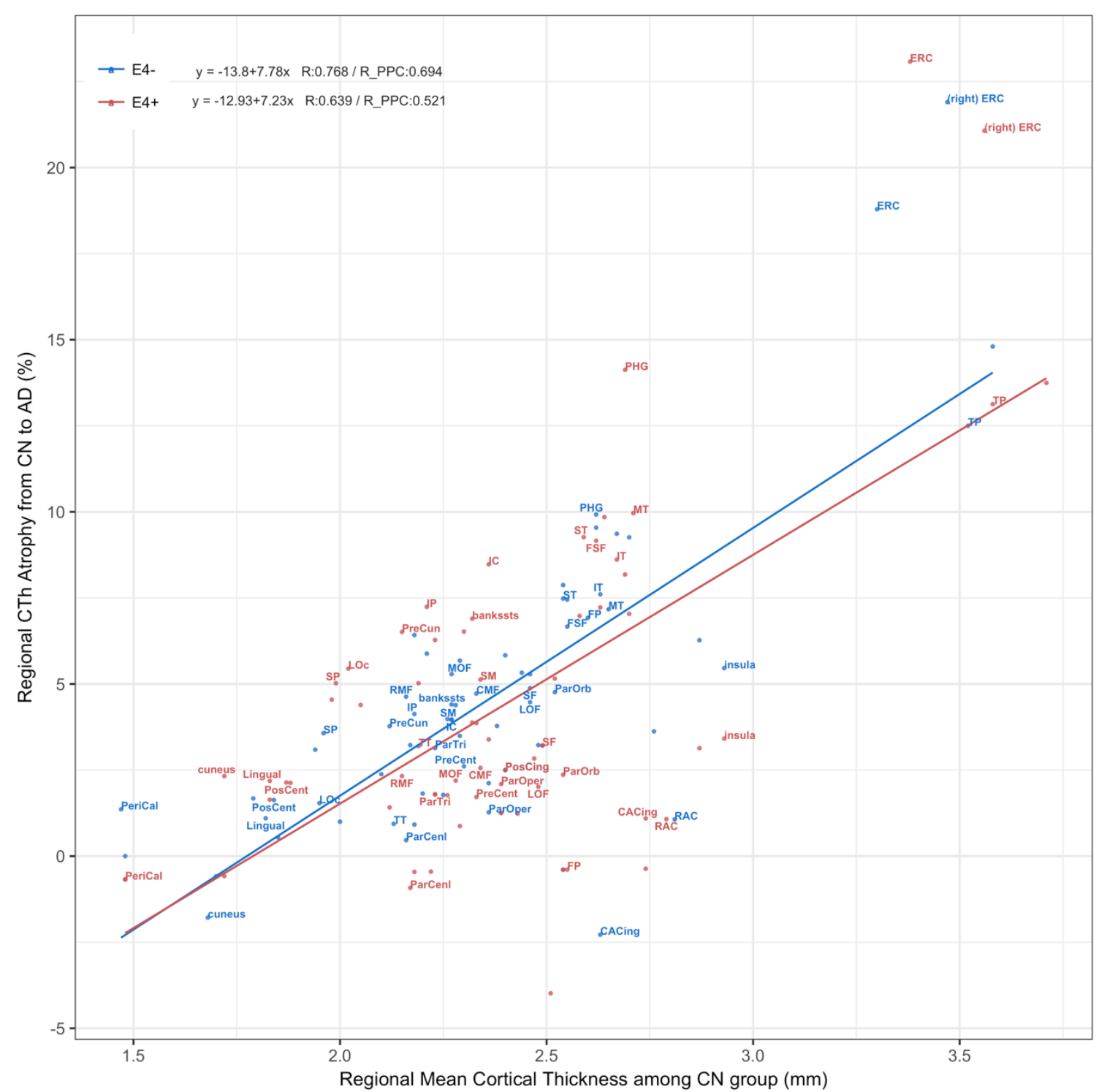

Fig. 21. Plot of $\mathrm{rCTh}^{\mathrm{CN}}$ and $\% \mathrm{CTh}^{\mathrm{Diff}}$ with the estimated linear regression model for the $\mathrm{E} 4+$ and $\mathrm{E} 4$ groups. $\mathrm{R}$ is the correlation coefficient for the Pearson correlation; R_PPC is the correlation coefficient for the Pearson Partial Correlation, adjusting for rSUVRCN. Abbreviations: bankssts, banks of the superior temporal sulcus; CAC, caudal anterior cingulate; CMF, caudal middle frontal; ERC, entorhinal cortex; IP, inferior parietal; IT, inferior temporal; IC, isthmus cingulate; LO, lateral occipital; LOF, lateral orbitofrontal; MOF, medial orbitofrontal; MT, middle temporal; PHG, parahippocampal; POP, pars opercularis; $\mathrm{POB}$, pars orbitalis; PTG, pars triangularis; PC, posterior cingulate; RAC, rostral anterior cingulate; RMF, rostral middle frontal; SF, superior frontal; SP, superior parietal; ST, superior temporal; SM, supramarginal; FP, frontal pole; TP, temporal pole; TT, transverse temporal. Except for the ERC, only ROIs in the left hemisphere were labeled since the similar patterns are observed in the right hemisphere. 
Table 23. Associations of $\mathrm{rCTh}^{\mathrm{CN}}$ with $\% \mathrm{CTh}^{\text {Diff }}$ before and after separating out the effect of $\mathrm{rSUVR}^{\mathrm{CN}}$

\begin{tabular}{lllll}
\hline & \multicolumn{2}{l}{ Linear Regression Model $^{\mathbf{a}}$} & $\begin{array}{l}\text { Pearson } \\
\text { Correlation }\end{array}$ & PPC $^{\mathbf{b}}$ \\
\cline { 2 - 3 } & Intercept & $\mathrm{rCTh}^{\mathrm{CN}}$ & & \\
\hline E4+ & & & & \\
Coefficient & -12.931 & 7.228 & 0.639 & 0.521 \\
Pvalue $^{\mathrm{c}}$ & $5.10 \mathrm{e}-06 * * *$ & $4.49 \mathrm{e}-09 * * *$ & $4.487 \mathrm{e}-09 * * *$ & $6.245 \mathrm{e}-06^{* * *}$ \\
\hline E4- & & & & \\
Coefficient & -13.803 & 7.779 & 0.768 & 0.694 \\
Pvalue & $7.49 \mathrm{e}-10 * * *$ & $2.25 \mathrm{e}-14 * * *$ & $2.255 \mathrm{e}-14 * * *$ & $7.748 \mathrm{e}-11 * * *$ \\
\hline
\end{tabular}

${ }^{\mathrm{a}}$ Linear regression model: $\% C T h^{D i f f}=\alpha+\beta_{r C T h} r C T h^{C N}$

${ }^{\mathrm{b}} \mathrm{PPC}$, Pearson Partial Correlation of $\mathrm{rCTh}^{\mathrm{CN}}$ and regional $\% \mathrm{CTh}^{\text {Diff }}$, correcting for $\mathrm{rSUVR}^{\mathrm{CN}}$

${ }^{c}$ p-value: ${ }^{*} \mathrm{p}<.05 ; * * \mathrm{p}<.01 ; * * * \mathrm{p}<.001$

\subsubsection{Effects of $\operatorname{rCTh}^{\mathrm{CN}}$ and $\mathrm{rSUVR}^{\mathrm{CN}}$ on $\% \mathrm{CTh}^{\mathrm{Diff}}$}

When including the interaction term $\mathrm{rCTh}^{\mathrm{CN}} \times \mathrm{rSUVR}^{\mathrm{CN}}$ in the multiple linear regression model $\% C T h^{D i f f}=\alpha+\beta_{r C T h} r C T h^{C N}+\beta_{r S U V R} r S U V R^{C N}+\beta_{C T h \cdot S U V R} r C T h^{C N} \times r S U V R^{C N}$, all variance inflation factors (VIFs) were larger than $10(85.8 / 101.8,30.4 / 30.2$, and 69.0/81.2 for $\mathrm{rCTh}^{\mathrm{CN}}, \mathrm{rSUVR}^{\mathrm{CN}}$, and $\mathrm{rCTh}^{\mathrm{CN}} \times \mathrm{rSUVR}^{\mathrm{CN}}$, and for E4+/E4-, respectively), indicating a very high level of multicollinearity. Thus, we excluded the interaction term in the linear regression study.

As depicted in Table 24, both $\mathrm{rCTh}^{\mathrm{CN}}$ and $\mathrm{rSUVR}^{\mathrm{CN}}$ were independent significant predictors of $\% \mathrm{CTh}^{\text {Diff }}$ in both E4+ and E4- subjects. Specifically, standardized beta coefficients showed greater explanatory power for $\mathrm{rCTh}^{\mathrm{CN}}$ than $\mathrm{rSUVR}^{\mathrm{CN}}$ in both $\mathrm{E} 4+$ $\left(\beta_{\mathrm{rCTh}}=2.357, \beta_{\mathrm{rSUVR}}=-1.563\right)$ and $\mathrm{E} 4-\left(\beta_{\mathrm{rCTh}}=2.594, \beta_{\mathrm{rSUVR}}=-1.399\right)$ groups 
The results shown in Fig. 22 and Table 24 reveal a negative relationship between the $\mathrm{rSUVR}^{\mathrm{CN}}$ and the $\% \mathrm{CTh}^{\text {Diff }}$, which was opposite to the $\mathrm{rCTh}^{\mathrm{CN}}$. Specifically, regions with the greatest $\% \mathrm{CTh}^{\text {Diff }}$ were aggregated in regions such as ERC $(23.08 \% / 18.79 \%$, for E4+/E4- group), parahippocampal gyrus $(14.13 \% / 9.92 \%)$, and temporal pole $(13.13 \% / 12.50 \%)$, which also had the greatest $\mathrm{rCTh}^{\mathrm{CN}}(3.38 \mathrm{~mm} / 3.30 \mathrm{~mm}$, $2.69 \mathrm{~mm} / 2.62 \mathrm{~mm}, 3.58 \mathrm{~mm} / 3.52 \mathrm{~mm}$, for ERC, parahippocampal gyrus and temporal pole, respectively). However, these regions tended to have the lowest $\operatorname{rSUVR}^{\mathrm{CN}}(0.89 / 0.87$, 0.96/0.92, 0.94/0.88). In contrast, $\mathrm{rCTh}^{\mathrm{CN}}$ was lowest in the pericalcarine, cuneus, postcentral, precuneus, and superior parietal regions, which also had the least $\% \mathrm{CTh}^{\text {Diff }}$ but the highest rSUVR ${ }^{\mathrm{CN}}$ (Table 21 and Table 22).

Table 24. Effects of $\mathrm{rCTh}^{\mathrm{CN}}$ and $\mathrm{rSUVR}^{\mathrm{CN}}$ on $\% \mathrm{CTh}^{\text {Diff a }}$

\begin{tabular}{|c|c|c|c|c|}
\hline & Intercept & $\mathrm{rCTh}^{\mathrm{CN}}$ & rSUVR $^{\mathrm{CN}}$ & adjust $R^{2}$ \\
\hline \multicolumn{5}{|l|}{$\overline{\text { E4+ }}$} \\
\hline Coefficients & 5.71 & $2.14^{\mathrm{b}}$ & -1.53 & 0.458 \\
\hline p-value ${ }^{c}$ & $<2 \mathrm{e}-16 * * *$ & $1.36 \mathrm{e}-05 * * *$ & $0.00133 * *$ & $8.28 \mathrm{e}-10 * * *$ \\
\hline \multicolumn{5}{|l|}{ E4- } \\
\hline \multirow{2}{*}{$\begin{array}{l}\text { Coefficients } \\
\text { p-value }\end{array}$} & 4.66 & 2.27 & -1.35 & 0.598 \\
\hline & $<2 \mathrm{e}-16 * * *$ & $4.08 \mathrm{e}-08 * * *$ & $0.000445 * * *$ & $5.01 \mathrm{e}-14 * * *$ \\
\hline \multicolumn{5}{|c|}{${ }^{\mathrm{a}}$ Multiple regression model: $\% C T h^{D i f f}=\alpha+\beta_{r C T h} r C T h^{C N}+\beta_{r S U V R} r S U V R^{C N}$} \\
\hline \multicolumn{5}{|c|}{$\begin{array}{l}{ }^{\mathrm{b}} \text { Using normalized values of } \mathrm{rCTh}^{\mathrm{CN}} \text { and } \mathrm{rSUVR}^{\mathrm{CN}} \text { (mean }=0 \text { and } \mathrm{SD}=1 \text { ) in the model, thus, the } \\
\text { coefficient is the adjusted beta weight. }\end{array}$} \\
\hline
\end{tabular}




\subsubsection{Associations between $\mathrm{rCTh}^{\mathrm{CN}}$ and Regional $\% \mathrm{CTh}^{\text {Diff }}$ for gSUVR+ and gSUVR- Subjects}

The obtained correlation coefficients were 0.613 for the gSUVR+ group and 0.831 for the gSUVR- group, which showed significant difference using the Fisher's z-test $\quad(z=-$ 2.724; $\mathrm{p}=0.0065)$ (Fig. 23).

\subsection{Discussion}

The regional $\mathrm{CTh}$ has been found to vary considerably across regions in human and primate brains, with primary sensory cortices being thinner than motor regions, which in turn are thinner than association cortices [151]. Normal aging is accompanied by global as well as regional structural changes. Both $\mathrm{CTh}$ and volumetric measures have demonstrated that the prefrontal cortex and to a lesser extent the parietal cortex, are sensitive to age-related decline $[149,152,153]$. In the current study, the age-related change in brain atrophy was accounted for using the age-equivalent $\mathrm{CN}$ and the $\mathrm{AD}$ group from the ADNI sample to examine those factors that pertained to selective vulnerability for neurodegeneration associated with Alzheimer's disease.

Our main findings are that: (1) regions with the greatest $\mathrm{CTh}$ at the $\mathrm{CN}$ stage are aggregated in regions which have been found to be most vulnerable to neurodegeneration in $\mathrm{AD}$, namely regions in the medial temporal lobe, including the temporal pole, ERC, parahippocampal gyrus, fusiform and the middle and inferior temporal gyrus; (2) regions with the lowest $\mathrm{CTh}$ in the $\mathrm{CN}$ stage were aggregated in regions which tend to be least 
vulnerable to neurodegeneration, namely the pericalcarine region, cuneus and the postcentral gyrus; (3) \%CTh ${ }^{\text {Diff }}$ was positively correlated to $\mathrm{rCTh}^{\mathrm{CN}}$, even after adjusting for the effect of rSUVR ${ }^{\mathrm{CN}}$; (4) $\mathrm{rCTh}^{\mathrm{CN}}$ showed greater explanatory power for $\% \mathrm{CTh}^{\text {Diff }}$ than $\operatorname{rSUVR}^{\mathrm{CN}}$; (5) there were no overall effect of the APOE4 genotype on the association between the $\mathrm{rCTh}^{\mathrm{CN}}$ and $\% \mathrm{CTh}^{\mathrm{Diff}}$; (6) the global $\mathrm{A} \beta$ load negative patients showed stronger effect on the association between the $\mathrm{rCTh}^{\mathrm{CN}}$ and $\% \mathrm{CTh}^{\text {Diff }}$ than the amyloid positive subjects.

Regions such as the ERC and the temporal pole had CTh which were between 3.3 and $4 \mathrm{~mm}$ in the $\mathrm{CN}$ stage and this $\mathrm{CTh}$ was reduced by $12 \%$ to $24 \%$ in the $\mathrm{AD}$ stage; whereas regions such as the pericalcarine region and the postcentral gyrus had CTh which were between 1.5 to $2 \mathrm{~mm}$ in the $\mathrm{CN}$ stage and were reduced by 0 to $5 \%$ in the $\mathrm{AD}$ stage (Table 21, Table 22, and Fig 21). These findings suggest that greater $\mathrm{CTh}$ in the $\mathrm{CN}$ stage may be a potential marker of greater vulnerability to the subsequent neurodegeneration due to $\mathrm{AD}$.

These findings initially appear divergent with a study by Sabuncu et al. on the ADNI population, which examined the association of a polygenic risk score to $\mathrm{CTh}$ in $\mathrm{CN}$ subjects and showed that a higher polygenic risk score was associated with decreased $\mathrm{CTh}$ among seven AD vulnerable regions [154]. However, a further examination of the associations between the polygenic risk score and $\mathrm{CTh}$ in individual studied regions, showed that among these seven regions, the region which is most vulnerable to neurodegeneration (23.78\% thinning from $\mathrm{CN}$ to $\mathrm{AD}$ for $\mathrm{E} 4+$ in our sample) (Table 21), 
namely the ERC, which has the greatest $\mathrm{CTh}$ among the seven region, showed no association to the polygenic risk score. In contrast, the posterior cingulate region, which was the only individual region showing a significant association between $\mathrm{CTh}$ and the polygenic risk score, was the least vulnerable of the seven regions to neurodegeneration $\left(\% \mathrm{CTh}^{\text {Diff }}=2.5 \%\right.$ for $\left.\mathrm{E} 4+\right)$ and had the least CTh (Table 21$)$. As such, the results of the study by Sabuncu et al are not inconsistent with the notion that greater CTh in the $\mathrm{CN}$ stage is associated with greater vulnerability to neurodegeneration.

The medial temporal lobe regions, including the ERC and parahippocampal gyrus receive highly processed input from every sensory modality, as well as input relating to ongoing cognitive processes. This information remains at least partially segregated among the different sensory visual, auditory olfactory, gustatory and tactile information from the neocortex and distribute it in a coded fashion to the hippocampus. The superficial layers of the ERC project to the dentate gyrus and hippocampus, with Layer II projecting primarily to the dentate gyrus and hippocampal region $\mathrm{CA} 3$, and layer III projecting primarily to hippocampal region CA1 and the subiculum. Spatially sensitive cells in layer II of the ERC have a crucial role in many spatially complex operations, including navigation and judging speed and accuracy of movements [155-159]. 


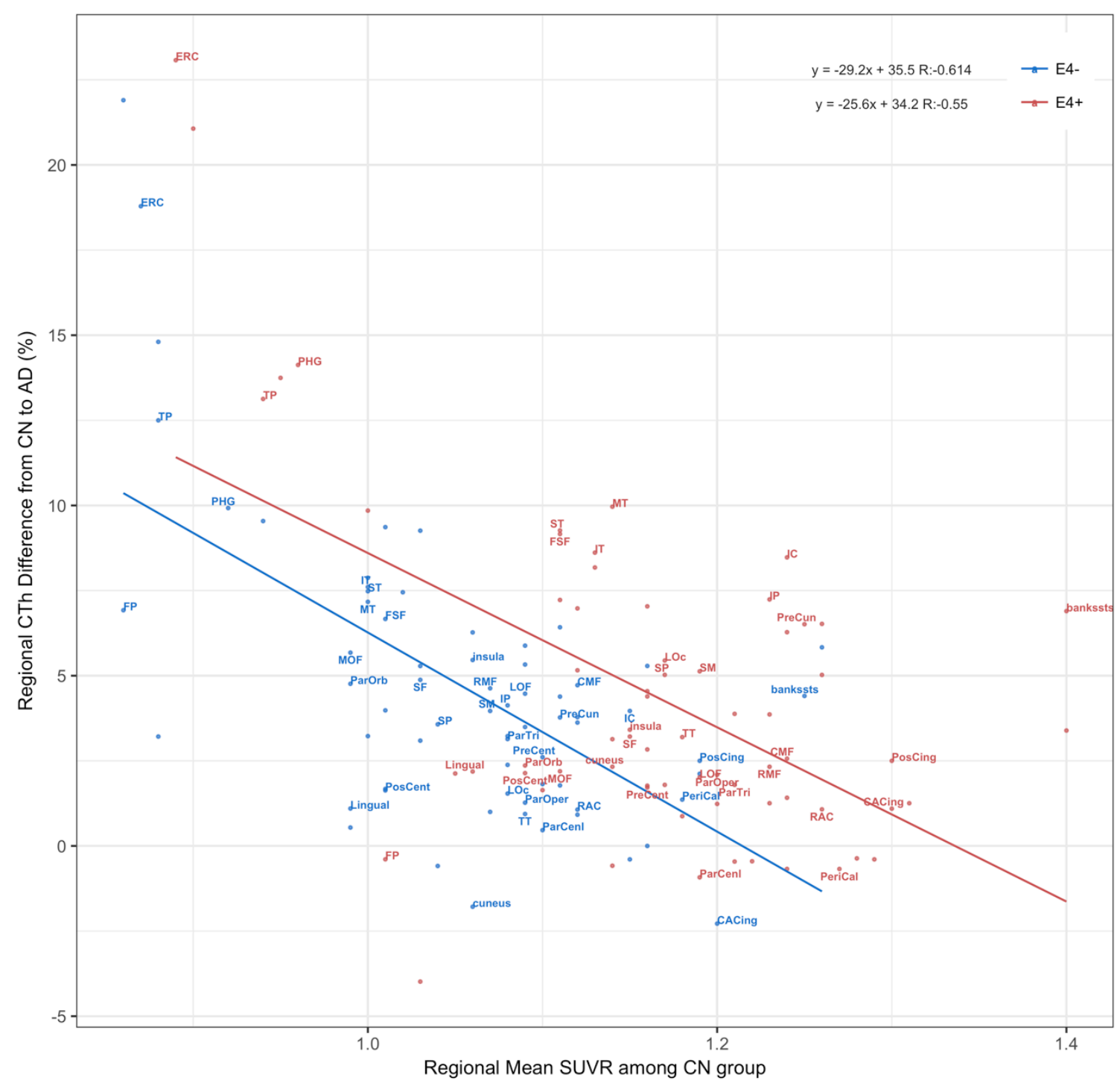

Fig. 22. Plot of rSUVR ${ }^{\mathrm{CN}}$ and $\% \mathrm{CTh}^{\mathrm{Diff}}$ with the estimated linear regression model for the $\mathrm{E} 4+$ and $\mathrm{E} 4$ groups. R is the correlation coefficient for the Pearson correlation, R_PPC is the correlation coefficient for the Pearson Partial Correlation, correcting for the rCThCN. Only ROIs in the left hemisphere were labeled, since results were similar for the left and the right hemisphere. Abbreviations: bankssts, banks of the superior temporal sulcus; CAC, caudal anterior cingulate; CMF, caudal middle frontal; ERC, entorhinal cortex; IP, inferior parietal; IT, inferior temporal; IC, isthmus cingulate; LO, lateral occipital; LOF, lateral orbitofrontal; MOF, medial orbitofrontal; MT, middle temporal; PHG, parahippocampal; POP, pars opercularis; $\mathrm{POB}$, pars orbitalis; PTG, pars triangularis; PC, posterior cingulate; RAC, rostral anterior cingulate; RMF, rostral middle frontal; SF, superior frontal; SP, superior parietal; ST, superior temporal; SM, supramarginal; FP, frontal pole; TP, temporal pole; TT, transverse temporal. 


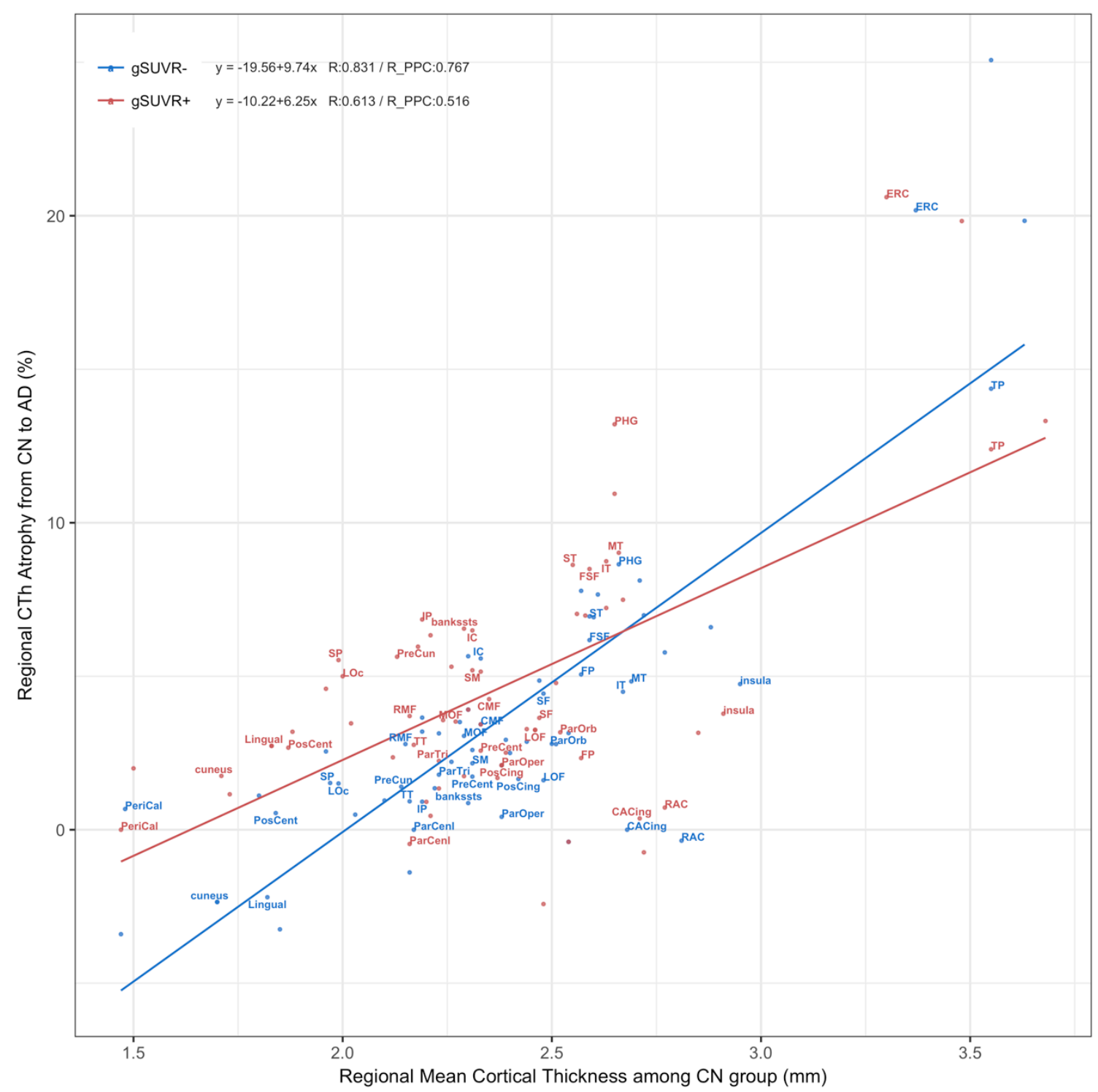

Fig. 23. Plot of $\mathrm{rCTh}^{\mathrm{CN}}$ and $\% \mathrm{CTh}^{\text {Diff }}$ with the estimated linear regression model for the global amyloid positive (gSUVR+) and negative (gSUVR-) groups. $\mathrm{R}$ is the correlation coefficient for the Pearson correlation; R_PPC is the correlation coefficient for the Pearson Partial Correlation, adjusting for rSUVRCN. Only ROIs in the left hemisphere were labeled. Abbreviations: bankssts, banks of the superior temporal sulcus; CAC, caudal anterior cingulate; CMF, caudal middle frontal; ERC, entorhinal cortex; IP, inferior parietal; IT, inferior temporal; IC, isthmus cingulate; LO, lateral occipital; LOF, lateral orbitofrontal; MOF, medial orbitofrontal; MT, middle temporal; PHG, parahippocampal; POP, pars opercularis; POB, pars orbitalis; PTG, pars triangularis; PC, posterior cingulate; RAC, rostral anterior cingulate; RMF, rostral middle frontal; SF, superior frontal; SP, superior parietal; ST, superior temporal; $\mathrm{SM}$, supramarginal; FP, frontal pole; TP, temporal pole; TT, transverse temporal. 
Greater CTh in the ERC and perirhinal cortex most likely represents the greater number of neurons, synapses and complexity of operations which are routinely performed by these brain regions [160]. Dickerson et al. showed that greater CTh in medial temporal regions was associated with better performance on verbal memory tasks and greater activation in these regions on functional MRI scans, in elderly CN subjects [161]. Progression of neurodegenerative pathology appears to occur preferentially in brain regions with the greatest connectivity and trafficking. For example, Seeley et al. have shown a direct link between intrinsic connectivity and gray matter structure across healthy individuals and have described nodes within each functional network which "exhibited tightly correlated gray matter volumes". They concluded that their findings suggest that "human neural networks can be defined by synchronous baseline activity, and selective vulnerability to neurodegenerative illness" [162]. The aggregation of misfolded disease proteins arises inside small, selectively vulnerable neuron populations in certain brain regions [163-165] and, as evidenced in human spongiform encephalopathies, conformational changes in misfolded prion protein results in disease propagation along the most active transsynaptic connections [166]. Neuropathological [11], neuroimaging [167, 168], and transgenic animal models [169], all suggest that neurodegeneration may relate to neural network dysfunction [168, 170], and selective vulnerability of specific brain regions may represent their status as hubs in very active networks.

In this study we have also shown that regions, such as the ERC, which show the greatest propensity for neurodegeneration has the lowest regional $\mathrm{A} \beta$ load, whereas many regions 
with relatively high $A \beta$ deposition, show the lowest propensity for atrophy. Although using multiple regression analyses, $A \beta$ burden was found to have a significant effect on neurodegeneration [80], this effect was much smaller in comparison to the association of $\mathrm{CTh}$ in the $\mathrm{CN}$ stage with neurodegeneration. Specifically, the most AD prone regions, such as ERC, parahippocampal, and temporal pole [129-132], had the greatest atrophy and the greatest baseline $\mathrm{CTh}$, but the least $\mathrm{A} \beta$ load. Conversely, other $\mathrm{AD}$ vulnerable regions, such as posterior cingulate, precuneus, inferior parietal, and isthmus cingulate, experienced lower atrophy but the $A \beta$ burden in these regions was higher. This discrepancy between regional $\mathrm{A} \beta$ deposition and regional reduction of $\mathrm{CTh}$ in patients with AD has been described previously [171]. These findings are consistent with the prevailing view that $A \beta$ deposition initiates or acts as a catalyst for the neurodegenerative process but that the correlation of such process with tau-protein deposition and tau associated neurofibrillary tangles concentration is much stronger than that with $\mathrm{A} \beta$ deposition [172].

In addition, the significant association of larger $\mathrm{rCTh}^{\mathrm{CN}}$ and greater atrophy after ruling out the effect of regional $A \beta$ burden agrees with the findings in a previous study [136], which may add credence to the belief that $A \beta$ does not play a leading role in brain atrophy [172]. Instead, tau pathology, inflammation, or disturbance in axonal transport processes may contribute to the ongoing neurodegeneration. However, the impact of APOE4 carrier status on neurodegeneration was not significant in comparison to the risk associated with regional CTh. Both the E4+ and E4- subjects showed a strong association between $\mathrm{CTh}$ in the $\mathrm{CN}$ stage and the propensity for neurodegeneration (Fig 21). 
The main weakness of this study is that it is a cross-sectional analysis. Unfortunately, in the ADNI cohort it is unlikely that a sufficient number of $\mathrm{CN}$ individuals will progress to $\mathrm{AD}$ dementia to actually relate original cortical thickness to rate of neurodegenerative regions in AD-related areas longitudinally, especially when accounting for the APOE4 carrier status and the availability of AV45 PET scans corresponding to the original MRI.

Longitudinal studies conducted on clinically normal individuals who have progressed to $\mathrm{AD}$ or were $\mathrm{A} \beta$ positive at baseline, have shown that mean $\mathrm{CTh}$ of $\mathrm{AD}$ vulnerable regions at baseline are thinner in comparison to the same regions among individuals who did not progress to $\mathrm{AD}$, or were $\mathrm{A} \beta$ negative at baseline. However, unlike the current investigation, these studies do not directly address the important question of whether CTh among individual regions is related to vulnerability to neurodegeneration $[79,173]$. The main strength of this cross-sectional study is the availability of $A \beta$ biomarkers and APOE genotypes in this ADNI cohort, which allow various factors to be assessed in this analysis of selective vulnerability. Besides, considering that aging and $\mathrm{AD}$ might have overlapping effects on the atrophy in specific cortical regions [174, 175], the study based on the age equivalent $\mathrm{CN}$ and $\mathrm{AD}$ groups superimposed the $\mathrm{AD}$-specific changes to the age related progressive atrophy.

\subsection{Conclusion}

In retrospect, in the present study, there is evidence for a strong positive association between $\mathrm{rCTh}^{\mathrm{CN}}$ and the severity of neurodegeneration (\%CTh$\left.{ }^{\text {Diff }}\right)$ in all brain regions. While we do not have an explanation for the most selective vulnerability for 
neurodegeneration of brain regions having the greatest $\mathrm{CTh}$ in the $\mathrm{CN}$ stage, we propose that, among other factors, greater CTh is associated with higher synaptic density, greater connectivity and complexity of function, which are factors that have been associated with a greater propensity to neurodegeneration. Other factors, such as disturbance in axonal transport, or inflammatory processes, in the presence of plaques and neurofibrillary tangles may also play a role in mediating the interaction between greater $\mathrm{CTh}$, greater connectivity and complexity of function and propensity for neurodegeneration. Our finding also show that regional $\mathrm{A} \beta$ load has a comparatively small but significant impact, but that APOE4 genotype and global A $\beta$ load had no significant impact on the propensity for neurodegeneration. 


\section{REFERENCES}

[1] R. A. Sperling, P. S. Aisen, L. A. Beckett, D. A. Bennett, S. Craft, A. M. Fagan, et al., "Toward defining the preclinical stages of Alzheimer's disease: recommendations from the National Institute on Aging-Alzheimer's Association workgroups on diagnostic guidelines for Alzheimer's disease," Alzheimers Dement, vol. 7, pp. 280-92, May 2011.

[2] M. S. Albert, S. T. DeKosky, D. Dickson, B. Dubois, H. H. Feldman, N. C. Fox, et al., "The diagnosis of mild cognitive impairment due to Alzheimer's disease: Recommendations from the National Institute on Aging-Alzheimer's Association workgroups on diagnostic guidelines for Alzheimer's disease," Alzheimers \& Dementia, vol. 7, pp. 270-279, May 2011.

[3] G. M. McKhann, D. S. Knopman, H. Chertkow, B. T. Hyman, C. R. Jack, C. H. Kawas, et al., "The diagnosis of dementia due to Alzheimer's disease: Recommendations from the National Institute on Aging-Alzheimer's Association workgroups on diagnostic guidelines for Alzheimer's disease," Alzheimers \& Dementia, vol. 7, pp. 263-269, May 2011.

[4] C. R. Jack, M. S. Albert, D. S. Knopman, G. M. McKhann, R. A. Sperling, M. C. Carrillo, et al., "Introduction to the recommendations from the National Institute on Aging-Alzheimer's Association workgroups on diagnostic guidelines for Alzheimer's disease," Alzheimers \& Dementia, vol. 7, pp. 257-262, May 2011.

[5] A. Alzheimer's, "2012 Alzheimer's disease facts and figures," Alzheimers Dement, vol. 8, pp. 131-68, 2012.

[6] A. Assoc, "Alzheimer's Association Report 2015 Alzheimer's disease facts and figures," Alzheimers \& Dementia, vol. 11, pp. 332-384, Mar 2015.

[7] L. E. H. Jennifer Weuve, Paul A. Scherr, Denis A. Evans, "Deaths in the United States among persons with Alzheimer's disease (2010-2050)," Alzheimers Dement, vol. 10, p. 7, March 2014.

[8] D. J. Selkoe, "The molecular pathology of Alzheimer's disease," Neuron, vol. 6, pp. 487-98, Apr 1991.

[9] B. T. Hyman, C. H. Phelps, T. G. Beach, E. H. Bigio, N. J. Cairns, M. C. Carrillo, et al., "National Institute on Aging-Alzheimer's Association guidelines for the neuropathologic assessment of Alzheimer's disease," Alzheimers Dement, vol. 8, pp. 113, Jan 2012.

[10] C. R. Jack, Jr., D. S. Knopman, W. J. Jagust, R. C. Petersen, M. W. Weiner, P. S. Aisen, et al., "Tracking pathophysiological processes in Alzheimer's disease: an updated hypothetical model of dynamic biomarkers," Lancet Neurol, vol. 12, pp. 207-16, Feb 2013. 
[11] H. Braak and E. Braak, "Neuropathological Staging of Alzheimer-Related Changes," Acta Neuropathologica, vol. 82, pp. 239-259, 1991.

[12] T. GomezIsla, H. L. West, G. W. Rebeck, S. D. Harr, J. H. Growdon, J. J. Locascio, et al., "Clinical and pathological correlates of apolipoprotein E epsilon 4 in Alzheimer's disease," Annals of Neurology, vol. 39, pp. 62-70, Jan 1996.

[13] A. Drzezga, T. Grimmer, G. Henriksen, M. Muehlau, R. Perneczky, I. Miederer, et al., "Effect of APOE genotype on amyloid plaque load and gray matter volume in Alzheimer disease," Neurology, vol. 72, pp. 1487-1494, Apr 282009.

[14] A. M. Cataldo, C. M. Peterhoff, J. C. Troncosco, T. Gomez-Isla, B. T. Hyman, and R. A. Nixon, "Endocytic pathway abnormalities precede amyloid beta deposition in sporadic Alzheimer's disease and Down syndrome - Differential effects of APOE genotype and presenilin mutations," American Journal of Pathology, vol. 157, pp. 277286, Jul 2000.

[15] M. M. Mielke, M. M. Machulda, C. E. Hagen, T. J. Christianson, R. O. Roberts, D. S. Knopman, et al., "Influence of amyloid and APOE on cognitive performance in a late middle-aged cohort," Alzheimers \& Dementia, vol. 12, pp. 281-291, Mar 2016.

[16] J. Kim, J. M. Basak, and D. M. Holtzman, "The Role of Apolipoprotein E in Alzheimer's Disease," Neuron, vol. 63, pp. 287-303, Aug 132009.

[17] K. Horsburgh, M. O. McCarron, F. White, and J. A. R. Nicoll, "The role of apolipoprotein E in Alzheimer's disease, acute brain injury and cerebrovascular disease: evidence of common mechanisms and utility of animal models," Neurobiology of Aging, vol. 21, pp. 245-255, Mar-Apr 2000.

[18] S. G. Potkin, G. Guffanti, A. Lakatos, J. A. Turner, F. Kruggel, J. H. Fallon, et al., "Hippocampal Atrophy as a Quantitative Trait in a Genome-Wide Association Study Identifying Novel Susceptibility Genes for Alzheimer's Disease," Plos One, vol. 4, Aug 7 2009.

[19] Y. He, C. Li, Y. Yang, Y. Z. Li, Y. Wang, H. Yang, et al., "Meta-analysis of the rs2075650 polymorphism and risk of Alzheimer disease," Aging Clinical and Experimental Research, vol. 28, pp. 805-811, Oct 2016.

[20] D. Harold, R. Abraham, P. Hollingworth, R. Sims, A. Gerrish, M. L. Hamshere, et al., "Genome-wide association study identifies variants at CLU and PICALM associated with Alzheimer's disease (vol 41, pg 1088, 2009)," Nature Genetics, vol. 41, pp. 11561156 , Oct 2009.

[21] M. W. Logue, M. Schu, B. N. Vardarajan, J. Buros, R. C. Green, R. C. P. Go, et al., "A Comprehensive Genetic Association Study of Alzheimer Disease in African Americans," Archives of Neurology, vol. 68, pp. 1569-1579, Dec 2011. 
[22] K. R. Murphy, S. M. Landau, K. R. Choudhury, C. A. Hostage, K. S. Shpanskaya, H. I. Sair, et al., "Mapping the effects of ApoE4, age and cognitive status on 18Fflorbetapir PET measured regional cortical patterns of beta-amyloid density and growth," Neuroimage, vol. 78, pp. 474-80, Sep 2013.

[23] A. Drzezga, T. Grimmer, G. Henriksen, M. Muhlau, R. Perneczky, I. Miederer, et al., "Effect of APOE genotype on amyloid plaque load and gray matter volume in Alzheimer disease," Neurology, vol. 72, pp. 1487-94, Apr 282009.

[24] A. S. Fleisher, K. Chen, X. Liu, N. Ayutyanont, A. Roontiva, P. Thiyyagura, et al., "Apolipoprotein E epsilon4 and age effects on florbetapir positron emission tomography in healthy aging and Alzheimer disease," Neurobiol Aging, vol. 34, pp. 1-12, Jan 2013.

[25] R. A. Sperling, P. S. Aisen, L. A. Beckett, D. A. Bennett, S. Craft, A. M. Fagan, et al., "Toward defining the preclinical stages of Alzheimer's disease: Recommendations from the National Institute on Aging-Alzheimer's Association workgroups on diagnostic guidelines for Alzheimer's disease," Alzheimers \& Dementia, vol. 7, pp. 280-292, May 2011.

[26] C. Li, D. A. Loewenstein, R. Duara, M. Cabrerizo, W. Barker, M. Adjouadi, et al., "The Relationship of Brain Amyloid Load and APOE Status to Regional Cortical Thinning and Cognition in the ADNI Cohort," J Alzheimers Dis, vol. 59, pp. 1269-1282, 2017.

[27] C. Fang, C. Li, M. Cabrerizo, A. Barreto, J. Andrian, D. A. Loewenstein, et al., "A Novel Gaussian Discriminant Analysis-based Computer Aided Diagnosis System for Screening Different Stages of Alzheimer's Disease," presented at the IEEE 17th International Conference on Bioinformatics and Bioengineering, Washington D.C, 2017.

[28] C. Fang, C. Li, M. Cabrerizo, A. Barreto, J. Andrian, D. A. Loewenstein, et al., "A Gaussian Discriminant Analysis-based Generative Learning Algorithm for the Early Diagnosis of Mild Cognitive Impairment in Alzheimer's Disease," in 2017 IEEE International Conference on Bioinformatics and Biomedicine, Kansas City, MO, 2017.

[29] H. I. Suk, S. W. Lee, D. G. Shen, and A. s. D. Neuroimaging, "Deep sparse multitask learning for feature selection in Alzheimer's disease diagnosis," Brain Structure \& Function, vol. 221, pp. 2569-2587, Jun 2016.

[30] B. Jie, D. Q. Zhang, B. Cheng, D. G. Shen, and A. s. D. Neuroimaging, "Manifold Regularized Multitask Feature Learning for Multimodality Disease Classification," Human Brain Mapping, vol. 36, pp. 489-507, Feb 2015.

[31] H. Guo, F. Zhang, J. J. Chen, Y. Xu, and J. Xiang, "Machine Learning Classification Combining Multiple Features of A Hyper-Network of fMRI Data in Alzheimer's Disease," Frontiers in Neuroscience, vol. 11, Nov 212017. 
[32] C. Davatzikos, Y. Fan, X. Wu, D. Shen, and S. M. Resnick, "Detection of prodromal Alzheimer's disease via pattern classification of magnetic resonance imaging," Neurobiology of Aging, vol. 29, pp. 514-523, Apr 2008.

[33] S. Alam, G. R. Kwon, J. I. Kim, and C. S. Park, "Twin SVM-Based Classification of Alzheimer's Disease Using Complex Dual-Tree Wavelet Principal Coefficients and LDA," Journal of Healthcare Engineering, 2017.

[34] M. Lopez, J. Ramirez, J. M. Gorriz, D. Salas-Gonzalez, I. Alvarez, F. Segovia, et al., "Automatic tool for Alzheimer's disease diagnosis using PCA and Bayesian classification rules," Electronics Letters, vol. 45, pp. 389-390, Apr 92009.

[35] S. Tripathi, S. H. Nozadi, M. Shakeri, and S. Kadoury, "Sub-Cortical Shape Morphology and Voxel-Based Features for Alzheimer's Disease Classification," 2017 Ieee 14th International Symposium on Biomedical Imaging (Isbi 2017), pp. 991-994, 2017.

[36] L. Khedher, I. A. Illan, J. M. Gorriz, J. Ramirez, A. Brahim, and A. Meyer-Baese, "Independent Component Analysis-Support Vector Machine-Based Computer-Aided Diagnosis System for Alzheimer's with Visual Support," Int J Neural Syst, vol. 27, p. 1650050, May 2017.

[37] M. Shakeri, H. Lombaert, and S. Kadoury, "Classification of Alzheimer's Disease Using Discriminant Manifolds of Hippocampus Shapes," Machine Learning Meets Medical Imaging, vol. 9487, pp. 65-73, 2015.

[38] S. Singh, A. Srivastava, L. Mi, R. J. Caselli, K. Chen, D. Goradia, et al., "Deep Learning based Classification of FDG-PET Data for Alzheimers Disease Categories," Proc SPIE Int Soc Opt Eng, vol. 10572, Oct 2017.

[39] R. Guerrero, R. Wolz, A. W. Rao, D. Rueckert, and Adni, "Manifold population modeling as a neuro-imaging biomarker: Application to ADNI and ADNI-GO," Neuroimage, vol. 94, pp. 275-286, Jul 12014.

[40] F. Li, L. Tran, K. H. Thung, S. Ji, D. Shen, and J. Li, "A Robust Deep Model for Improved Classification of AD/MCI Patients," IEEE J Biomed Health Inform, vol. 19, pp. 1610-6, Sep 2015.

[41] R. Li, W. Zhang, H. I. Suk, L. Wang, J. Li, D. Shen, et al., "Deep learning based imaging data completion for improved brain disease diagnosis," Med Image Comput Comput Assist Interv, vol. 17, pp. 305-12, 2014.

[42] H. I. Suk, S. W. Lee, D. Shen, and I. Alzheimer's Disease Neuroimaging, "Deep sparse multi-task learning for feature selection in Alzheimer's disease diagnosis," Brain Struct Funct, vol. 221, pp. 2569-87, Jun 2016. 
[43] M. Shakeri, H. Lombaert, S. Tripathi, and S. Kadoury, "Deep Spectral-Based Shape Features for Alzheimer's Disease Classification," Cham, 2016, pp. 15-24.

[44] A. Ortiz, J. Munilla, J. M. Gorriz, and J. Ramirez, "Ensembles of Deep Learning Architectures for the Early Diagnosis of the Alzheimer's Disease," Int J Neural Syst, vol. 26, p. 1650025, Nov 2016.

[45] F. C. Morabito, M. Campolo, N. Mammone, M. Versaci, S. Franceschetti, F. Tagliavini, et al., "Deep Learning Representation from Electroencephalography of EarlyStage Creutzfeldt-Jakob Disease and Features for Differentiation from Rapidly Progressive Dementia," International Journal of Neural Systems, vol. 27, Mar 2017.

[46] G. Prasad, S. H. Joshi, T. M. Nir, A. W. Toga, P. M. Thompson, and Adni, "Brain connectivity and novel network measures for Alzheimer's disease classification," Neurobiology of Aging, vol. 36, pp. S121-S131, Jan 2015.

[47] A. Soldan, C. Pettigrew, Y. Lu, M. C. Wang, O. Selnes, M. Albert, et al., "Relationship of medial temporal lobe atrophy, APOE genotype, and cognitive reserve in preclinical Alzheimer's disease," Human Brain Mapping, vol. 36, pp. 2826-2841, Jul 2015.

[48] R. S. Desikan, F. Segonne, B. Fischl, B. T. Quinn, B. C. Dickerson, D. Blacker, et al., "An automated labeling system for subdividing the human cerebral cortex on MRI scans into gyral based regions of interest," Neuroimage, vol. 31, pp. 968-980, Jul 12006.

[49] M. Jenkinson, C. F. Beckmann, T. E. Behrens, M. W. Woolrich, and S. M. Smith, "Fsl," Neuroimage, vol. 62, pp. 782-790, Aug 152012.

[50] L. Breiman, "Random forests," Machine Learning, vol. 45, pp. 5-32, Oct 2001.

[51] A. Meyer-Lindenberg and D. R. Weinberger, "Intermediate phenotypes and genetic mechanisms of psychiatric disorders," Nature Reviews Neuroscience, vol. 7, pp. 818-827, Oct 2006.

[52] R. Rasetti and D. R. Weinberger, "Intermediate phenotypes in psychiatric disorders," Current Opinion in Genetics \& Development, vol. 21, pp. 340-348, Jun 2011.

[53] Gottesman, II and T. D. Gould, "The endophenotype concept in psychiatry: etymology and strategic intentions," Am J Psychiatry, vol. 160, pp. 636-45, Apr 2003.

[54] E. J. Rose and G. Donohoe, "Brain vs Behavior: An Effect Size Comparison of Neuroimaging and Cognitive Studies of Genetic Risk for Schizophrenia," Schizophrenia Bulletin, vol. 39, pp. 518-526, May 2013.

[55] N. K. Batmanghelich, A. V. Dalca, M. R. Sabuncu, G. Polina, and Adni, "Joint modeling of imaging and genetics," Inf Process Med Imaging, vol. 23, pp. 766-77, 2013. 
[56] M. Vounou, E. Janousova, R. Wolz, J. L. Stein, P. M. Thompson, D. Rueckert, et al., "Sparse reduced-rank regression detects genetic associations with voxel-wise longitudinal phenotypes in Alzheimer's disease," Neuroimage, vol. 60, pp. 700-716, Mar 2012.

[57] N. Filippini, A. Rao, S. Wetten, R. A. Gibson, M. Borrie, D. Guzman, et al., "Anatomically-distinct genetic associations of APOE epsilon4 allele load with regional cortical atrophy in Alzheimer's disease," Neuroimage, vol. 44, pp. 724-8, Feb 012009.

[58] S. G. Potkin, J. A. Turner, G. Guffanti, A. Lakatos, J. H. Fallon, D. D. Nguyen, et al., "A Genome-Wide Association Study of Schizophrenia Using Brain Activation as a Quantitative Phenotype," Schizophrenia Bulletin, vol. 35, pp. 96-108, Jan 2009.

[59] J. H. Lee, R. Cheng, N. Graff-Radford, T. Foroud, R. Mayeux, and G. National Institute on Aging Late-Onset Alzheimer's Disease Family Study, "Analyses of the National Institute on Aging Late-Onset Alzheimer's Disease Family Study: implication of additional loci," Arch Neurol, vol. 65, pp. 1518-26, Nov 2008.

[60] Y. Benjamini and Y. Hochberg, "Controlling the False Discovery Rate - a Practical and Powerful Approach to Multiple Testing," Journal of the Royal Statistical Society Series B-Methodological, vol. 57, pp. 289-300, 1995.

[61] K. A. Le Cao, D. Rossouw, C. Robert-Granie, and P. Besse, "A sparse PLS for variable selection when integrating omics data," Stat Appl Genet Mol Biol, vol. 7, p. Article 35, 2008.

[62] E. Le Floch, V. Guillemot, V. Frouin, P. Pinel, C. Lalanne, L. Trinchera, et al., "Significant correlation between a set of genetic polymorphisms and a functional brain network revealed by feature selection and sparse Partial Least Squares," Neuroimage, vol. 63, pp. 11-24, Oct 152012.

[63] B. Fischl and A. M. Dale, "Measuring the thickness of the human cerebral cortex from magnetic resonance images," Proc Natl Acad Sci U S A, vol. 97, pp. 11050-5, Sep 262000 .

[64] B. B. Avants, N. J. Tustison, G. Song, P. A. Cook, A. Klein, and J. C. Gee, "A reproducible evaluation of ANTs similarity metric performance in brain image registration," Neuroimage, vol. 54, pp. 2033-44, Feb 12011.

[65] A. Klein and J. Tourville, "101 labeled brain images and a consistent human cortical labeling protocol," Front Neurosci, vol. 6, p. 171, 2012.

[66] J. A. K. Suykens and J. Vandewalle, "Least squares support vector machine classifiers," Neural Processing Letters, vol. 9, pp. 293-300, Jun 1999. 
[67] H. Chun and S. Keles, "Sparse partial least squares regression for simultaneous dimension reduction and variable selection," J R Stat Soc Series B Stat Methodol, vol. 72, pp. 3-25, Jan 2010.

[68] A. Bakkour, J. C. Morris, and B. C. Dickerson, "The cortical signature of prodromal AD: regional thinning predicts mild AD dementia," Neurology, vol. 72, pp. 1048-55, Mar 242009.

[69] Q. Zhou, F. Zhao, Z. P. Lv, C. G. Zheng, W. D. Zheng, L. Sun, et al., "Association between APOC1 Polymorphism and Alzheimer's Disease: A Case-Control Study and Meta-Analysis," Plos One, vol. 9, Jan 312014.

[70] R. Ferrari, Y. Wang, J. Vandrovcova, S. Guelfi, A. Witeolar, C. M. Karch, et al., "Genetic architecture of sporadic frontotemporal dementia and overlap with Alzheimer's and Parkinson's diseases," J Neurol Neurosurg Psychiatry, vol. 88, pp. 152-164, Feb 2017.

[71] Q. S. Li, A. R. Parrado, M. N. Samtani, V. A. Narayan, and I. Alzheimer's Disease Neuroimaging, "Variations in the FRA10AC1 Fragile Site and 15q21 Are Associated with Cerebrospinal Fluid Abeta1-42 Level," PLoS One, vol. 10, p. e0134000, 2015.

[72] C. Li, C. Fang, M. Cabrerizo, A. Barreto, J. Andrian, R. Duara, et al., "A Neuroimaging Feature Extraction Model for Imaging Genetics with Application to Alzheimer's Disease," in IEEE International Conference on Bio-Informatics and BioEngineering, Washington D.C, 2017.

[73] C. R. Jack, Jr., H. J. Wiste, S. D. Weigand, D. S. Knopman, P. Vemuri, M. M. Mielke, et al., "Age, Sex, and APOE epsilon4 Effects on Memory, Brain Structure, and beta-Amyloid Across the Adult Life Span," JAMA Neurol, vol. 72, pp. 511-9, May 2015.

[74] P. Vemuri, H. J. Wiste, S. D. Weigand, D. S. Knopman, L. M. Shaw, J. Q. Trojanowski, et al., "Effect of apolipoprotein E on biomarkers of amyloid load and neuronal pathology in Alzheimer disease," Ann Neurol, vol. 67, pp. 308-16, Mar 2010.

[75] R. J. Caselli, A. C. Dueck, D. Osborne, M. N. Sabbagh, D. J. Connor, G. L. Ahern, et al., "Longitudinal modeling of age-related memory decline and the APOE epsilon4 effect," N Engl J Med, vol. 361, pp. 255-63, Jul 162009.

[76] J. C. Morris, "The Clinical Dementia Rating (Cdr) - Current Version and Scoring Rules," Neurology, vol. 43, pp. 2412-2414, Nov 1993.

[77] M. F. Folstein, S. E. Folstein, and P. R. McHugh, "'Mini-mental state". A practical method for grading the cognitive state of patients for the clinician," J Psychiatr Res, vol. 12, pp. 189-98, Nov 1975. 
[78] A. Rey, $L^{\prime}$ examen clinique en psychologie. Paris: Presses universitaires de France, 1964.

[79] B. C. Dickerson, A. Bakkour, D. H. Salat, E. Feczko, J. Pacheco, D. N. Greve, et al., "The cortical signature of Alzheimer's disease: regionally specific cortical thinning relates to symptom severity in very mild to mild $\mathrm{AD}$ dementia and is detectable in asymptomatic amyloid-positive individuals," Cereb Cortex, vol. 19, pp. 497-510, Mar 2009.

[80] J. A. Becker, T. Hedden, J. Carmasin, J. Maye, D. M. Rentz, D. Putcha, et al., "Amyloid-beta associated cortical thinning in clinically normal elderly," Ann Neurol, vol. 69, pp. 1032-42, Jun 2011.

[81] A. D. Joshi, M. J. Pontecorvo, C. M. Clark, A. P. Carpenter, D. L. Jennings, C. H. Sadowsky, et al., "Performance Characteristics of Amyloid PET with Florbetapir F 18 in Patients with Alzheimer's Disease and Cognitively Normal Subjects," Journal of Nuclear Medicine, vol. 53, pp. 378-384, Mar 12012.

[82] D. A. Loewenstein, R. E. Curiel, M. T. Greig, R. M. Bauer, M. Rosado, D. Bowers, et al., "A Novel Cognitive Stress Test for the Detection of Preclinical Alzheimer Disease: Discriminative Properties and Relation to Amyloid Load," American Journal of Geriatric Psychiatry, vol. 24, pp. 804-813, Oct 2016.

[83] R Core Team, "R: A language and environment for statistical computing," ed. Vienna, Austria: R Foundation for Statistical Computing, 2017.

[84] J. Q. Trojanowski, H. Vandeerstichele, M. Korecka, C. M. Clark, P. S. Aisen, R. C. Petersen, et al., "Update on the biomarker core of the Alzheimer's Disease Neuroimaging Initiative subjects," Alzheimers \& Dementia, vol. 6, pp. 230-238, May 2010.

[85] A. S. Fleisher, K. Chen, X. Liu, A. Roontiva, P. Thiyyagura, N. Ayutyanont, et al., "Using positron emission tomography and florbetapir F18 to image cortical amyloid in patients with mild cognitive impairment or dementia due to Alzheimer disease," Arch Neurol, vol. 68, pp. 1404-11, Nov 2011.

[86] K. A. Ellis, Y. Y. Lim, K. Harrington, D. Ames, A. I. Bush, D. Darby, et al., "Decline in Cognitive Function over 18 Months in Healthy Older Adults with High Amyloid-beta," Journal of Alzheimers Disease, vol. 34, pp. 861-871, 2013.

[87] Y. Y. Lim, K. A. Ellis, D. Ames, D. Darby, K. Harrington, R. N. Martins, et al., "Abeta amyloid, cognition, and APOE genotype in healthy older adults," Alzheimers Dement, vol. 9, pp. 538-45, Sep 2013.

[88] J. L. Whitwell, N. Tosakulwong, S. D. Weigand, M. L. Senjem, V. J. Lowe, J. L. Gunter, et al., "Does amyloid deposition produce a specific atrophic signature in cognitively normal subjects?," Neuroimage-Clinical, vol. 2, pp. 249-257, 2013. 
[89] M. J. Grothe, M. Ewers, B. Krause, H. Heinsen, S. J. Teipel, and I. Alzheimer's Disease Neuroimaging, "Basal forebrain atrophy and cortical amyloid deposition in nondemented elderly subjects," Alzheimers Dement, vol. 10, pp. S344-53, Oct 2014.

[90] X. Da, J. B. Toledo, J. Zee, D. A. Wolk, S. X. Xie, Y. Ou, et al., "Integration and relative value of biomarkers for prediction of MCI to $\mathrm{AD}$ progression: spatial patterns of brain atrophy, cognitive scores, APOE genotype and CSF biomarkers," Neuroimage Clin, vol. 4, pp. 164-73, 2014.

[91] G. Chetelat, R. La Joie, N. Villain, A. Perrotin, V. de La Sayette, F. Eustache, et al., "Amyloid imaging in cognitively normal individuals, at-risk populations and preclinical Alzheimer's disease," Neuroimage-Clinical, vol. 2, pp. 356-365, 2013.

[92] S. Lista, F. G. Garaci, M. Ewers, S. Teipel, H. Zetterberg, K. Blennow, et al., "CSF Abeta1-42 combined with neuroimaging biomarkers in the early detection, diagnosis and prediction of Alzheimer's disease," Alzheimers Dement, vol. 10, pp. 38192, May 2014.

[93] R. Duara, D. A. Loewenstein, Q. Shen, W. Barker, E. Potter, D. Varon, et al., "Amyloid positron emission tomography with (18)F-flutemetamol and structural magnetic resonance imaging in the classification of mild cognitive impairment and Alzheimer's disease," Alzheimers Dement, vol. 9, pp. 295-301, May 2013.

[94] M. J. Grothe, S. J. Teipel, and A. D. N. Initia, "Spatial Patterns of Atrophy, Hypometabolism, and Amyloid Deposition in Alzheimer's Disease Correspond to Dissociable Functional Brain Networks," Human Brain Mapping, vol. 37, pp. 35-53, Jan 2016.

[95] L. G. Apostolova, K. S. Hwang, O. Kohannim, D. Avila, D. Elashoff, C. R. Jack, et al., "ApoE4 effects on automated diagnostic classifiers for mild cognitive impairment and Alzheimer's disease," Neuroimage-Clinical, vol. 4, pp. 461-472, 2014.

[96] Y. Liu, T. Paajanen, E. Westman, L. O. Wahlund, A. Simmons, C. Tunnard, et al., "Effect of APOE epsilon4 allele on cortical thicknesses and volumes: the AddNeuroMed study," J Alzheimers Dis, vol. 21, pp. 947-66, 2010.

[97] H. Lemaitre, F. Crivello, C. Dufouil, B. Grassiot, C. Tzourio, A. Alperovitch, et al., "No epsilon(4) gene dose effect on hippocampal atrophy in a large MRI database of healthy elderly subjects," Neuroimage, vol. 24, pp. 1205-1213, Feb 152005.

[98] S. Matura, D. Prvulovic, A. Jurcoane, D. Hartmann, J. Miller, M. Scheibe, et al., "Differential effects of the ApoE4 genotype on brain structure and function," Neuroimage, vol. 89, pp. 81-91, Apr 12014.

[99] M. Pievani, S. Galluzzi, P. M. Thompson, P. E. Rasser, M. Bonetti, and G. B. Frisoni, "APOE4 is associated with greater atrophy of the hippocampal formation in Alzheimer's disease," Neuroimage, vol. 55, pp. 909-919, Apr 12011. 
[100] M. Fouquet, F. L. Besson, J. Gonneaud, R. La Joie, and G. Chetelat, "Imaging Brain Effects of APOE4 in Cognitively Normal Individuals Across the Lifespan," Neuropsychology Review, vol. 24, pp. 290-299, Sep 2014.

[101] G. Chetelat and M. Fouquet, "Neuroimaging biomarkers for Alzheimer's disease in asymptomatic APOE4 carriers," Rev Neurol (Paris), vol. 169, pp. 729-36, Oct 2013.

[102] V. Heise, N. Filippini, A. J. Trachtenberg, S. Suri, K. P. Ebmeier, and C. E. Mackay, "Apolipoprotein E genotype, gender and age modulate connectivity of the hippocampus in healthy adults," Neuroimage, vol. 98, pp. 23-30, Sep 2014.

[103] L. Chang, M. Andres, J. Sadino, C. S. Jiang, H. Nakama, E. Miller, et al., "Impact of apolipoprotein E epsilon4 and HIV on cognition and brain atrophy: antagonistic pleiotropy and premature brain aging," Neuroimage, vol. 58, pp. 1017-27, Oct 152011.

[104] C. Pettigrew, A. Soldan, Y. Zhu, M. C. Wang, A. Moghekar, T. Brown, et al., "Cortical thickness in relation to clinical symptom onset in preclinical AD," Neuroimage Clin, vol. 12, pp. 116-22, 2016.

[105] H. Lin, R. Bhatia, and R. Lal, "Amyloid beta protein forms ion channels: implications for Alzheimer's disease pathophysiology," Faseb Journal, vol. 15, pp. 24332444, Nov 2001.

[106] S. Rosales-Corral, D. X. Tan, R. J. Reiter, M. Valdivia-Velazquez, J. P. AcostaMartinez, and G. G. Ortiz, "Kinetics of the neuroinflammation-oxidative stress correlation in rat brain following the injection of fibrillar amyloid-beta onto the hippocampus in vivo," J Neuroimmunol, vol. 150, pp. 20-8, May 2004.

[107] D. A. Butterfield, T. Reed, S. F. Newman, and R. Sultana, "Roles of amyloid beta-peptide-associated oxidative stress and brain protein modifications in the pathogenesis of Alzheimer's disease and mild cognitive impairment," Free Radic Biol Med, vol. 43, pp. 658-77, Sep 012007.

[108] K. Parameshwaran, M. Dhanasekaran, and V. Suppiramaniam, "Amyloid beta peptides and glutamatergic synaptic dysregulation," Exp Neurol, vol. 210, pp. 7-13, Mar 2008.

[109] S. L. Risacher, S. Kim, K. Nho, T. Foroud, L. Shen, R. C. Petersen, et al., "APOE effect on Alzheimer's disease biomarkers in older adults with significant memory concern," Alzheimers Dement, vol. 11, pp. 1417-29, Dec 2015.

[110] C. Li, D. A. Loewenstein, R. Duara, M. Cabrerizo, W. Barker, and M. Adjouadi, "Associations between regional amyloid load, cortical thickness, APOE genotype and cognition in ADNIGO/ADNI2 participants," presented at the Human Amyloid Imaging, Miami, FL, 2017. 
[111] K. Nho, S. L. Risacher, P. K. Crane, C. DeCarli, M. M. Glymour, C. Habeck, et al., "Voxel and surface-based topography of memory and executive deficits in mild cognitive impairment and Alzheimer's disease," Brain Imaging and Behavior, vol. 6, pp. 551-567, Dec 2012.

[112] K. W. Chen, J. B. S. Langbaum, A. S. Fleisher, N. Ayutyanont, C. Reschke, W. Lee, et al., "Twelve-month metabolic declines in probable Alzheimer's disease and amnestic mild cognitive impairment assessed using an empirically pre-defined statistical region-of-interest: Findings from the Alzheimer's Disease Neuroimaging Initiative," Neuroimage, vol. 51, pp. 654-664, Jun 2010.

[113] A. B. Wolf, J. Valla, G. Bu, J. Kim, M. J. LaDu, E. M. Reiman, et al., "Apolipoprotein E as a beta-amyloid-independent factor in Alzheimer's disease," Alzheimers Res Ther, vol. 5, p. 38, 2013.

[114] B. C. Dickerson, D. H. Salat, D. N. Greve, E. F. Chua, E. Rand-Giovannetti, D. M. Rentz, et al., "Increased hippocampal activation in mild cognitive impairment compared to normal aging and AD," Neurology, vol. 65, pp. 404-11, Aug 092005.

[115] R. W. Mahley, K. H. Weisgraber, and Y. Huang, "Apolipoprotein E4: a causative factor and therapeutic target in neuropathology, including Alzheimer's disease," Proc Natl Acad Sci U S A, vol. 103, pp. 5644-51, Apr 112006.

[116] S. L. Risacher, S. Kim, L. Shen, K. Nho, T. Foroud, R. C. Green, et al., "The role of apolipoprotein E (APOE) genotype in early mild cognitive impairment (E-MCI)," Frontiers in Aging Neuroscience, vol. 5, Apr 12013.

[117] T. Espeseth, L. T. Westlye, K. B. Walhovd, A. M. Fjell, T. Endestad, H. Rootwelt, et al., "Apolipoprotein E epsilon 4-related thickening of the cerebral cortex modulates selective attention," Neurobiology of Aging, vol. 33, pp. 304-U621, Feb 2012.

[118] G. Torres, P. McCall, C. Liu, M. Cabrerizo, and M. Adjouadi, "EEG processing: a many-core approach utilising the Intel single-chip cloud computer platform," International Journal of Embedded Systems, vol. 9, pp. 464-474, 2017.

[119] G. Chetelat, V. L. Villemagne, P. Bourgeat, K. E. Pike, G. Jones, D. Ames, et al., "Relationship between atrophy and beta-amyloid deposition in Alzheimer disease," Ann Neurol, vol. 67, pp. 317-24, Mar 2010.

[120] G. Chetelat, V. L. Villemagne, K. E. Pike, K. A. Ellis, D. Ames, C. L. Masters, et al., "Relationship between Memory Performance and beta-Amyloid Deposition at Different Stages of Alzheimer's Disease," Neurodegenerative Diseases, vol. 10, pp. 141144, 20122012.

[121] D. Tosun, N. Schuff, C. A. Mathis, W. Jagust, M. W. Weiner, and I. Alzheimer's Disease NeuroImaging, "Spatial patterns of brain amyloid-beta burden and atrophy rate associations in mild cognitive impairment," Brain, vol. 134, pp. 1077-88, Apr 2011. 
[122] P. T. Trzepacz, H. Hochstetler, P. Yu, P. Castelluccio, M. M. Witte, G. Dell'Agnello, et al., "Relationship of Hippocampal Volume to Amyloid Burden across Diagnostic Stages of Alzheimer's Disease," Dementia and Geriatric Cognitive Disorders, vol. 41, pp. 68-79, 20162016.

[123] V. Dore, V. L. Villemagne, P. Bourgeat, J. Fripp, O. Acosta, G. Chetelat, et al., "Cross-sectional and Longitudinal Analysis of the Relationship Between A beta Deposition, Cortical Thickness, and Memory in Cognitively Unimpaired Individuals and in Alzheimer Disease," Jama Neurology, vol. 70, pp. 903-911, Jul 2013.

[124] P. S. Insel, N. Mattsson, M. C. Donohue, R. S. Mackin, P. S. Aisen, C. R. Jack, Jr., et al., "The transitional association between beta-amyloid pathology and regional brain atrophy," Alzheimers \& Dementia, vol. 11, pp. 1171-1179, Oct 2015.

[125] J. L. Whitwell, N. Tosakulwong, S. D. Weigand, M. L. Senjem, V. J. Lowe, J. L. Gunter, et al., "Does amyloid deposition produce a specific atrophic signature in cognitively normal subjects?," Neuroimage Clin, vol. 2, pp. 249-57, 2013.

[126] Z. Qi, M. Goryawala, M. Cabrerizo, W. Barker, D. Loewenstein, R. Duara, et al., "Multivariate Analysis of structural MRI and PET (FDG and 18F-AV-45) for Alzheimer's disease and its prodromal stages," Conference proceedings : ... Annual International Conference of the IEEE Engineering in Medicine and Biology Society. IEEE Engineering in Medicine and Biology Society. Annual Conference, vol. 2014, pp. 1051-4, 2014-Aug 2014.

[127] G. Chetelat, V. L. Villemagne, P. Bourgeat, K. E. Pike, G. Jones, D. Ames, et al., "Relationship between Atrophy and beta-Amyloid Deposition in Alzheimer Disease," Annals of Neurology, vol. 67, pp. 317-324, Mar 2010.

[128] N. Filippini, A. Rao, S. Wetten, R. A. Gibson, M. Borrie, D. Guzman, et al., "Anatomically-distinct genetic associations of APOE epsilon 4 allele load with regional cortical atrophy in Alzheimer's disease," Neuroimage, vol. 44, pp. 724-728, Feb 12009.

[129] B. C. Dickerson, I. Goncharova, M. P. Sullivan, C. Forchetti, R. S. Wilson, D. A. Bennett, et al., "MRI-derived entorhinal and hippocampal atrophy in incipient and very mild Alzheimer's disease," Neurobiol Aging, vol. 22, pp. 747-54, Sep-Oct 2001.

[130] C. R. Jack, Jr., R. C. Petersen, Y. C. Xu, S. C. Waring, P. C. O'Brien, E. G. Tangalos, et al., "Medial temporal atrophy on MRI in normal aging and very mild Alzheimer's disease," Neurology, vol. 49, pp. 786-94, Sep 1997.

[131] R. J. Killiany, M. B. Moss, M. S. Albert, T. Sandor, J. Tieman, and F. Jolesz, "Temporal lobe regions on magnetic resonance imaging identify patients with early Alzheimer's disease," Arch Neurol, vol. 50, pp. 949-54, Sep 1993.

[132] H. Braak and E. Braak, "Neuropathological stageing of Alzheimer-related changes," Acta Neuropathol, vol. 82, pp. 239-59, 1991. 
[133] O. Hansson, H. Zetterberg, P. Buchhave, E. Londos, K. Blennow, and L. Minthon, "Association between CSF biomarkers and incipient Alzheimer's disease in patients with mild cognitive impairment: a follow-up study," Lancet Neurol, vol. 5, pp. 228-34, Mar 2006.

[134] N. Mattsson, D. Tosun, P. S. Insel, A. Simonson, C. R. Jack, L. A. Beckett, et al., "Association of brain amyloid-beta with cerebral perfusion and structure in Alzheimer's disease and mild cognitive impairment," Brain, vol. 137, pp. 1550-1561, May 2014.

[135] X. Long, L. Zhang, W. Liao, C. Jiang, B. Qiu, and I. Alzheimer's Disease Neuroimaging, "Distinct laterality alterations distinguish mild cognitive impairment and Alzheimer's disease from healthy aging: statistical parametric mapping with high resolution MRI," Hum Brain Mapp, vol. 34, pp. 3400-10, Dec 2013.

[136] G. Chetelat, V. L. Villemagne, K. E. Pike, J. C. Baron, P. Bourgeat, G. Jones, et al., "Larger temporal volume in elderly with high versus low beta-amyloid deposition," Brain, vol. 133, pp. 3349-58, Nov 2010.

[137] D. Iacono, W. R. Markesbery, M. Gross, O. Pletnikova, G. Rudow, P. Zandi, et al., "The Nun study: clinically silent $\mathrm{AD}$, neuronal hypertrophy, and linguistic skills in early life," Neurology, vol. 73, pp. 665-73, Sep 012009.

[138] M. A. Riudavets, D. Iacono, S. M. Resnick, R. O'Brien, A. B. Zonderman, L. J. Martin, et al., "Resistance to Alzheimer's pathology is associated with nuclear hypertrophy in neurons," Neurobiology of Aging, vol. 28, pp. 1484-1492, Oct 2007.

[139] J. Pegueroles, E. Vilaplana, V. Montal, F. Sampedro, D. Alcolea, M. CarmonaIragui, et al., "Longitudinal brain structural changes in preclinical Alzheimer's disease," Alzheimers Dement, Sep 282016.

[140] J. Fortea, R. Sala-Llonch, D. Bartres-Faz, A. Llado, C. Sole-Padulles, B. Bosch, et al., "Cognitively preserved subjects with transitional cerebrospinal fluid ss-amyloid 1-42 values have thicker cortex in Alzheimer's disease vulnerable areas," Biol Psychiatry, vol. 70, pp. 183-90, Jul 152011.

[141] B. M. Doherty, S. A. Schultz, J. M. Oh, R. L. Koscik, N. M. Dowling, T. E. Barnhart, et al., "Amyloid burden, cortical thickness, and cognitive function in the Wisconsin Registry for Alzheimer's Prevention," Alzheimers Dement (Amst), vol. 1, pp. 160-169, Jun 012015.

[142] A. Grothey, M. Shah, T. Yoshino, E. Van Cutsem, J. Taieb, R. Xu, et al., "Clinicopathological factors influence diagnostic accuracy of clinical $\mathrm{N}$ staging for early gastric cancer," Annals of Oncology, vol. 28, Jun 2017. 
[143] A. D. Cohen, J. C. Price, L. A. Weissfeld, J. James, B. L. Rosario, W. Bi, et al., "Basal cerebral metabolism may modulate the cognitive effects of Abeta in mild cognitive impairment: an example of brain reserve," J Neurosci, vol. 29, pp. 14770-8, Nov 252009.

[144] R. Ossenkoppele, C. Madison, H. Oh, M. Wirth, B. N. M. van Berckel, and W. J. Jagust, "Is Verbal Episodic Memory in Elderly with Amyloid Deposits Preserved Through Altered Neuronal Function?," Cerebral Cortex, vol. 24, pp. 2210-2218, Aug 2014.

[145] W. J. Jagust and E. C. Mormino, "Lifespan brain activity, beta-amyloid, and Alzheimer's disease," Trends in Cognitive Sciences, vol. 15, pp. 520-526, Nov 2011.

[146] S. Schreiber, S. M. Landau, A. Fero, F. Schreiber, W. J. Jagust, and A. s. D. N. Initi, "Comparison of Visual and Quantitative Florbetapir F 18 Positron Emission Tomography Analysis in Predicting Mild Cognitive Impairment Outcomes," Jama Neurology, vol. 72, pp. 1183-1190, Oct 2015.

[147] V. Camus, P. Payoux, L. Barre, B. Desgranges, T. Voisin, C. Tauber, et al., "Using PET with F-18-AV-45 (florbetapir) to quantify brain amyloid load in a clinical environment," European Journal of Nuclear Medicine and Molecular Imaging, vol. 39, pp. 621-631, Apr 2012.

[148] Z. S. Ji, R. D. Miranda, Y. M. Newhouse, K. H. Weisgraber, Y. D. Huang, and R. W. Mahley, "Apolipoprotein E4 potentiates amyloid beta peptide-induced lysosomal leakage and apoptosis in neuronal cells," Journal of Biological Chemistry, vol. 277, pp. 21821-21828, Jun 2002.

[149] H. Lemaitre, A. L. Goldman, F. Sambataro, B. A. Verchinski, A. MeyerLindenberg, D. R. Weinberger, et al., "Normal age-related brain morphometric changes: nonuniformity across cortical thickness, surface area and gray matter volume?," Neurobiol Aging, vol. 33, pp. 617 e1-9, Mar 2012.

[150] S. M. McGinnis, M. Brickhouse, B. Pascual, and B. C. Dickerson, "Age-Related Changes in the Thickness of Cortical Zones in Humans," Brain Topography, vol. 24, pp. 279-291, Oct 2011.

[151] F. Kruggel, M. K. Bruckner, T. Arendt, C. J. Wiggins, and D. Y. von Cramon, "Analyzing the neocortical fine-structure," Med Image Anal, vol. 7, pp. 251-64, Sep 2003.

[152] K. Juottonen, M. P. Laakso, R. Insausti, M. Lehtovirta, A. Pitkanen, K. Partanen, et al., "Volumes of the entorhinal and perirhinal cortices in Alzheimer's disease," Neurobiol Aging, vol. 19, pp. 15-22, Jan-Feb 1998. 
[153] A. M. Fjell, L. McEvoy, D. Holland, A. M. Dale, K. B. Walhovd, and A. s. D. N. Initi, "What is normal in normal aging? Effects of aging, amyloid and Alzheimer's disease on the cerebral cortex and the hippocampus," Progress in Neurobiology, vol. 117, pp. 20-40, Jun 2014.

[154] M. R. Sabuncu, R. L. Buckner, J. W. Smoller, P. H. Lee, B. Fischl, R. A. Sperling, et al., "The Association between a Polygenic Alzheimer Score and Cortical Thickness in Clinically Normal Subjects," Cerebral Cortex, vol. 22, pp. 2653-2661, Nov 2012.

[155] T. Hafting, M. Fyhn, S. Molden, M. B. Moser, and E. I. Moser, "Microstructure of a spatial map in the entorhinal cortex," Nature, vol. 436, pp. 801-806, Aug 112005.

[156] E. L. Hargreaves, G. Rao, I. Lee, and J. J. Knierim, "Major dissociation between medial and lateral entorhinal input to dorsal hippocampus," Science, vol. 308, pp. 17921794, Jun 172005.

[157] M. Fyhn, S. Molden, M. P. Witter, E. I. Moser, and M. B. Moser, "Spatial representation in the entorhinal cortex," Science, vol. 305, pp. 1258-64, Aug 272004.

[158] E. Kropff, J. E. Carmichael, M. B. Moser, and E. I. Moser, "Speed cells in the medial entorhinal cortex," Nature, vol. 523, pp. 419-24, Jul 232015.

[159] J. Jacobs, M. J. Kahana, A. D. Ekstrom, M. V. Mollison, and I. Fried, "A sense of direction in human entorhinal cortex," Proc Natl Acad Sci U S A, vol. 107, pp. 6487-92, Apr 062010.

[160] D. H. Geschwind and P. Rakic, "Cortical evolution: judge the brain by its cover," Neuron, vol. 80, pp. 633-47, Oct 302013.

[161] B. C. Dickerson, E. Fenstermacher, D. H. Salat, D. A. Wolk, R. P. Maguire, R. Desikan, et al., "Detection of cortical thickness correlates of cognitive performance: Reliability across MRI scan sessions, scanners, and field strengths," Neuroimage, vol. 39, pp. 10-8, Jan 012008.

[162] W. W. Seeley, R. K. Crawford, J. Zhou, B. L. Miller, and M. D. Greicius, "Neurodegenerative diseases target large-scale human brain networks," Neuron, vol. 62, pp. 42-52, Apr 162009.

[163] G. A. Graveland, R. S. Williams, and M. DiFiglia, "Evidence for degenerative and regenerative changes in neostriatal spiny neurons in Huntington's disease," Science, vol. 227, pp. 770-3, Feb 151985.

[164] B. T. Hyman, G. W. Van Hoesen, A. R. Damasio, and C. L. Barnes, "Alzheimer's disease: cell-specific pathology isolates the hippocampal formation," Science, vol. 225, pp. 1168-70, Sep 141984. 
[165] W. W. Seeley, D. A. Carlin, J. M. Allman, M. N. Macedo, C. Bush, B. L. Miller, et al., "Early frontotemporal dementia targets neurons unique to apes and humans," Ann Neurol, vol. 60, pp. 660-7, Dec 2006.

[166] J. R. Scott, D. Davies, and H. Fraser, "Scrapie in the central nervous system: neuroanatomical spread of infection and Sinc control of pathogenesis," J Gen Virol, vol. 73 ( Pt 7), pp. 1637-44, Jul 1992.

[167] M. D. Greicius, G. Srivastava, A. L. Reiss, and V. Menon, "Default-mode network activity distinguishes Alzheimer's disease from healthy aging: Evidence from functional MRI," Proceedings of the National Academy of Sciences of the United States of America, vol. 101, pp. 4637-4642, Mar 302004.

[168] R. L. Buckner, A. Z. Snyder, B. J. Shannon, G. LaRossa, R. Sachs, A. F. Fotenos, et al., "Molecular, structural, and functional characterization of Alzheimer's disease: Evidence for a relationship between default activity, amyloid, and memory," Journal of Neuroscience, vol. 25, pp. 7709-7717, Aug 242005.

[169] J. J. Palop, J. Chin, E. D. Roberson, J. Wang, M. T. Thwin, N. Bien-Ly, et al., "Aberrant excitatory neuronal activity and compensatory remodeling of inhibitory hippocampal circuits in mouse models of Alzheimer's disease," Neuron, vol. 55, pp. 697711, Sep 62007.

[170] J. J. Palop, J. Chin, and L. Mucke, "A network dysfunction perspective on neurodegenerative diseases," Nature, vol. 443, pp. 768-773, Oct 192006.

[171] R. La Joie, A. Perrotin, L. Barre, C. Hommet, F. Mezenge, M. Ibazizene, et al., "Region-Specific Hierarchy between Atrophy, Hypometabolism, and beta-Amyloid (A beta) Load in Alzheimer's Disease Dementia," Journal of Neuroscience, vol. 32, pp. 16265-16273, Nov 142012.

[172] B. T. Hyman, "Amyloid-Dependent and Amyloid-Independent Stages of Alzheimer Disease," Archives of Neurology, vol. 68, pp. 1062-1064, Aug 2011.

[173] C. Pettigrew, A. Soldan, Y. Zhu, M. C. Wang, A. Moghekar, T. Brown, et al., "Cortical thickness in relation to clinical symptom onset in preclinical AD," NeuroimageClinical, vol. 12, pp. 116-122, 2016.

[174] A. M. Fjell, L. McEvoy, D. Holland, A. M. Dale, K. B. Walhovd, and I. Alzheimer's Disease Neuroimaging, "Brain changes in older adults at very low risk for Alzheimer's disease," J Neurosci, vol. 33, pp. 8237-42, May 082013.

[175] A. Bakkour, J. C. Morris, D. A. Wolk, and B. C. Dickerson, "The effects of aging and Alzheimer's disease on cerebral cortical anatomy: specificity and differential relationships with cognition," Neuroimage, vol. 76, pp. 332-44, Aug 012013. 
VITA

\section{CHUNFEI LI}

Sep 2005 - Jun 2009 B.S., Mathematics and Applied Mathematics, Shijiazhuang Railway Institute

Sep 2011 - Mar 2017 M.S., Computer Software Engineering, Beihang University

Feb 2012 - Aug 2012 IOS Developer, Huangfeng Corporation, Beijing, China

Aug 2012 - Dec 2013 M.S., Computer Engineering, Florida International University

Jan 2014 - Apr 2018 Ph.D., Electrical Engineering, Florida International University

May 2016 - Aug 2016 Data Science Intern, Rokk3r Labs, Miami, Florida

Sep 2017 - Nov 2017 Software Engineer Intern, Facebook, New York, NY

\section{PUBLICATIONS AND PRESENTATIONS}

C. Li, D.A. Loewenstein, R. Duara, M. Cabrerizo, W. Barker, M. Adjouadi, "The Relationship of Brain Amyloid Load and APOE Status to Regional Cortical Thinning and Cognition in the ADNI Cohort", Journal of Alzheimer's Disease. 2017; Vol. 59(4), pp. 1269-1282, DOI: 10.3233/JAD-170286, PMID: 28731444.

C. Li, C. Fang, M. Cabrerizo, A. Barreto, J. Andrian, R. Duara, D.A. Loewenstein, M. Adjouadi, "Regional Image Features Model for the Genome-wide Association Study of Alzheimer's Disease", IEEE Journal of Biomedical Health Informatics (accepted)

C. Li, C. Fang, M. Cabrerizo, A. Barreto, J. Andrian, D.A. Loewenstein, R. Duara, M. Adjouadi, "Diverging association patterns of the regional amyloid load, cortical thickness and APOE genotype in the progression of Alzheimer's disease", International Journal of Computational Biology and Drug Design (IJCBDD) (accepted with revision)

C. Li, Duara R, Loewenstein DA, Cabrerizo M, Barker W, Adjouadi M, "Greater Regional Cortical Thickness is Associated With Selective Vulnerability to Atrophy in Alzheimer's Disease, Independent of Amyloid Load and APOE Genotypet", Journal of Alzheimer's Disease (under review)

C. Li, C. Fang, M. Cabrerizo, A. Barreto, J. Andrian, N. Rishe, D. Loewenstein, R. Duara, M. Adjouadi, "Enhanced Region-based Neuroimaging Features for the Early Detection of Alzheimer's Disease", International Journal of Neural Systems (under review)

C. Li, C. Fang, M. Cabrerizo, A. Barreto, J. Andrian, N. Rishe, D. Loewenstein, R. Duara, M. Adjouadi, "Diverging association patterns of regional cortical atrophy with global and with regional amyloid deposition” Human Brain Mapping (under review)

C. Li, C. Fang, M. Adjouadi, M. Cabrerizo, A. Barreto, J. Andrian, R. Duara, D. Loewenstein. "A Neuroimaging Feature Extraction Model for Imaging Genetics with 
Application to Alzheimer's Disease". 2017 IEEE 17th International Conference on Bioinformatics and Bioengineering (BIBE), pp. 15-20, DOI: 10.1109/BIBE.2017.00010, Washington DC, October 23-25, 2017.

C. Li, C. Fang, M. Cabrerizo, A. Barreto, J. Andrian, D. Loewenstein, R. Duara, M. Adjouad. "Pattern analysis of the interaction of regional amyloid load, cortical thickness and APOE genotype in the progression of Alzheimer's disease". IEEE International Conference on Bioinformatics and Biomedicine (BIBM), pp. 2171-2176, 2017, DOI: 10.1109/BIBM.2017.8217994

C. Li, Q. Zhou, M. Goryawala, D.A. Loewenstein, W. Barker, R. Duara, M. Adjouadi. "Investigating the Utility of FDG and AV45 PET in both Two-classification and Multiclassification of Alzheimer's Disease and Its Prodromal Stages", The 9th Human Amyloid Imaging. pp. 117, 2015.

C. Li, Q. Zhou, M. Adjouadi. "Correlation Regional Amyloid Load and Cortical Thickness in Alzheimer's Disease". GWAS 2016, Florida International University, Miami, FL, Mar 28-29, 2016

C. Li, R. Duara, D.A. Loewenstein, M. Cabrerizo, W. Barker, M. Adjouadi. "Interaction of regional amyloid load, cortical thickness and APOE genotype in Alzheimer's disease". BIOT Symposium, Provo, Utah, Dec 8-9, 2016

C. Li, R. Duara, D.A. Loewenstein, M. Cabrerizo, W. Barker, A. Barreto, M. Adjouadi. "Genome-wide association study of Alzheimer's disease using supervised neuroimaging features as intermediate phenotypes". BIOT Symposium, Provo, Utah, Dec 8-9, 2016

C. Li, R. Duara, D.A. Loewenstein, M. Cabrerizo, W. Barker, M. Adjouadi. "Greater Regional Cortical Thickness is Associated With Selective Vulnerability to Atrophy in Alzheimer's Disease, Independent of Regional Amyloid Load". Human Amyloid Imaging, pp. 141-143, Jan 14, 2017.

C. Li, D.A. Loewenstein, R. Duara, M. Cabrerizo, W. Barker, M. Adjouadi. "Associations between regional amyloid load, cortical thickness, APOE genotype and cognition in ADNIGO/ADNI2 participants" Human Amyloid Imaging, pp. 221-224, 2017.

C. Li, R. Duara, D.A. Loewenstein, M. Cabrerizo, W. Barker, M. Adjouadi. "Optimal Neuroimaging Measures for Tracking Alzheimer's Disease Progression". Alzheimer's and Dementia 13(7): P438-P440, DOI10.1016/j.jalz.2017.06.437

C. Fang, C. Li, M. Cabrerizo, A. Barreto, J. Andrian, N. Rishe, D.A. Loewenstein, R. Duara, M. Adjouadi. "A Gaussian Discriminant Analysis-based Machine Learning Algorithm for the Classification of Mild Cognitive Impairment in Alzheimer's disease". BIOT Symposium conference, Provo, Utah, Dec 8-9, 2016

C. Fang, C. Li, M. Cabrerizo, A. Barreto, J. Andrian, N. Rishe, D.A. Loewenstein, R. Duara, M. Adjouadi, "Gaussian Discriminant Analysis-Based Dual High-Dimensional Decision Spaces for the Diagnosis of Mild Cognitive Impairment in Alzheimer's Disease", International Journal of Neural Systems. (accepted) 
C. Fang, C. Li, M. Cabrerizo, A. Barreto, J. Andrian, D. Loewenstein, R. Duara, and M. Adjouadi "A Novel Gaussian Discriminant Analysis-based Computer Aided Diagnosis System for Screening Different Stages of Alzheimer's Disease", IEEE 17th International Conference on Bioinformatics and Bioengineering (BIBE), pp. 279-282, Washington DC, October 23-25, 2017.

C. Fang, C. Li, M. Cabrerizo, A. Barreto, J. Andrian, D. Loewenstein, R. Duara, M. Adjouadi, "A Gaussian discriminant analysis-based generative learning algorithm for the early diagnosis of mild cognitive impairment in Alzheimer's disease", IEEE International Conference on Bioinformatics and Biomedicine (BIBM), pp. 538 - 542, 2017.

C. Fang, P. Janwattanapong, C. Li, M. Adjouadi, "A Global Feature Extraction Model for the Effective Computer Aided Diagnosis of Mild Cognitive Impairment Using Structural MRI Images", NIPS 2017 Machine Learning for Health Workshop (ML4H)

G. Lizarraga, C. Li, M. Cabrerizo, W. Barker, D.A. Loewenstein, R. Duara, M. Adjouadi, "A Neuroimaging Web Services Interface Design as a Cyber Physical System for Medical Imaging and Data Management in Brain Research", JMIR Medical Informatics. (accepted)

D. A. Loewenstein, R. E. Curiel, S. DeKosky, M. Rosselli, R. Bauer, M. Grieg-Custo, A. Penate, C. Li, G. Lizaragga, T. Golde, M. Adjouadi, R. Duara, "Recovery from Proactive Semantic Interference and MRI Volume: A Replication and Extension Study", Journal of Alzheimer's Disease. 2017; 59(1):131-139. DOI: 10.3233/JAD-170276, PMID: 28598850.

X. Wang, C. Li, M. Goryawala, M. Cabrerizo, M. Adjouadi, "Integrated Multimodal Registration Technique for Medical Imaging", Medical Image Analysis (under review) 